\title{
Total Synthesis of Naturally Occurring 5,7,8-Trioxygenated Homoisoflavonoids
}

\author{
Sangil Kwon, Sanha Lee, Myunghoe Heo, Bit Lee, Xiang Fei, Timothy W. Corson, and Seung-Yong Seo*
}

Cite This: ACS Omega 2020, 5, 11043-11057

Read Online

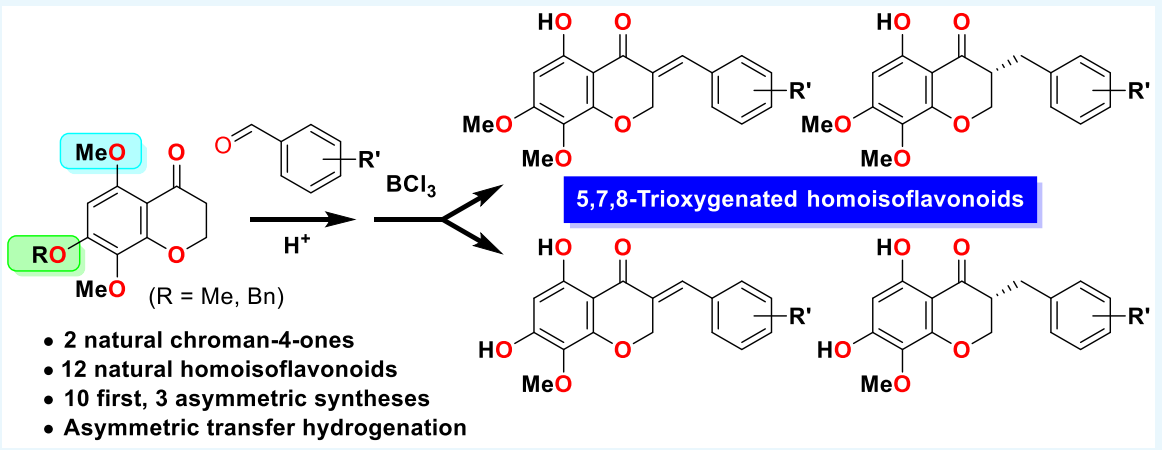

ABSTRACT: Homoisoflavonoids are in the subclass of the larger family of flavonoids but have one more alkyl carbon than flavonoids. Among them, 5,7,8-trioxygenated homoisoflavonoids have not been extensively studied for synthesis and biological evaluation. Our current objective is to synthesize 2 5,7,8-trioxygenated chroman-4-ones and 12 5,7,8-trioxygenated homoisoflavonoids that have been isolated from the plants Bellevalia eigii, Drimiopsis maculata, Ledebouria graminifolia, Eucomis autumnalis, Eucomis punctata, Eucomis pallidiflora, Chionodoxa luciliae, Muscari comosum, and Dracaena cochinchinensis. For this purpose, 1,3,4,5-tetramethoxybenzene and $4^{\prime}$-benzyloxy-2',3'-dimethoxy- $6^{\prime}$-hydroxyacetophenone were used as starting materials. Asymmetric transfer hydrogenation using Noyori's $\mathrm{Ru}$ catalyst provided 5,7,8-trioxygenated-3-benzylchroman-4-ones with Rconfiguration in high yield and enantiomeric excess. By selective deprotection of homoisoflavonoids using $\mathrm{BCl}_{3}$, the total synthesis of natural products including 10 first syntheses and three asymmetric syntheses has been completed, and three isomers of the reported dracaeconolide B could be provided. Our research on 5,7,8-trioxygenated homoisoflavonoids would be useful for the synthesis of related natural products and pharmacological applications.

\section{INTRODUCTION}

Homoisoflavonoids are a subclass of the larger family of flavonoids, with a 16-carbon skeleton that has 1 more carbon (C9 position) than flavonoids and isoflavonoids (Figure 1). They have a structure including two aromatic rings and a

(a) Sappanin-type<smiles>c1ccc(CC2COc3ccccc3C2)cc1</smiles>

(b) Scillascillins-type<smiles>c1ccc2c(c1)CC1(CO2)Cc2ccccc21</smiles>

(c) Brazilin-type

(d) Caesalpin-type

(c) Protosappanin-type<smiles>c1ccc2c(c1)CC1COc3ccccc3C21</smiles><smiles>C1=CC2OCC3Cc4ccccc4C2(C=C1)C3</smiles><smiles>CC1COc2ccccc2-c2ccccc2C1</smiles>

heterocycle containing oxygen. As some review articles dealt with the natural origins and structures of homoisoflavonoids, they are mainly found in Asparagaceae and Fabaceae families and are rarely seen in Polygonaceae, Portulacaceae, Orchidaceae, and Gentianaceae families. ${ }^{1,2}$ Homoisoflavonoids have been reported to have various biological activities, including antiangiogenic, antibacterial, antimutagenic, antioxidant, and anti-inflammatory effects. ${ }^{3}$ Initially, the structural types of homoisoflavonoids were categorized into four scaffolds-3benzylchroman-4-one, 3-benzylidenechroman-4-one, 3-benzyl3-hydroxychroman-4-one, and scillascillins-by du Toit's group. ${ }^{4}$ As some isolated natural products were not included in such a category of homoisoflavonoids, the newly classified

Received: March 2, 2020

Accepted: April 23, 2020

Published: May 6, 2020

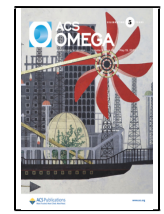

Figure 1. Categories of homoisoflavonoids. 
<smiles>COc1cc(O)c2c(c1OC)OCCC2=O</smiles>

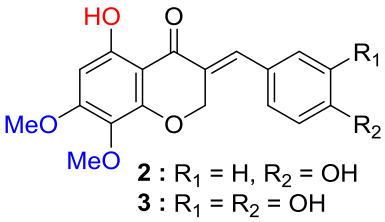

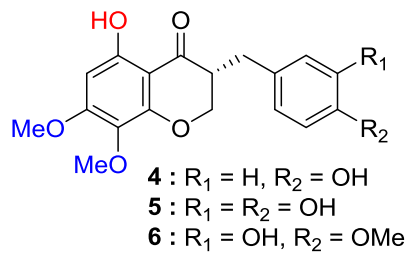<smiles>COc1c(O)cc(O)c2c1OCCC2=O</smiles>

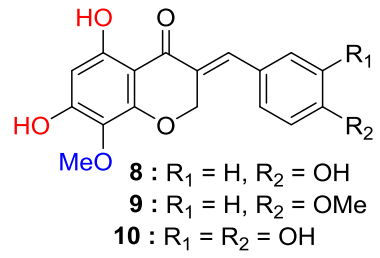

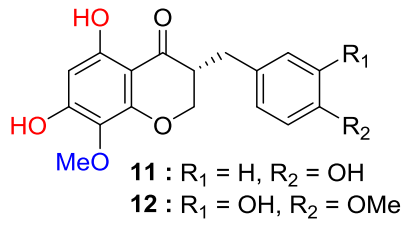<smiles>COc1cc(OC)c2c(c1OC)OC[C@H](Cc1ccc(O)cc1)C2=O</smiles><smiles>COc1cc(O)c(OC)c2c1C[C@H](Cc1ccc(O)cc1)CO2</smiles>

Figure 2. Naturally occurring 5,7,8-trioxygenated chromanones and homoisoflavonoids.

five types of homoisoflavonoids are as follows: sappanin-type, scillascillin-type, brazilin-type, caesalpin-type, and protosappanin-type (Figure 1). ${ }^{1}$

Among them, sappanin-type natural products are the most isolated and studied. The methoxy and hydroxy groups are mainly substituted in the A ring and the $\mathrm{B}$ ring, respectively. In the A ring, naturally occurring homoisoflavonoids having mono- to tetra-substituents have been reported so far. In particular, we have been interested in the total synthesis and biological activities of 5,6,7-trisubstituted homoisoflavonoids. Naturally occurring homoisoflavonoids that contain either 5,7dihydroxy-6-methoxy or 7-hydroxy-5,6-dimethoxy groups in the A ring have been synthesized by us. ${ }^{5,6}$ Cremastranone and its synthetic analogs having the 5,6,7-trimethoxy group in the A ring were biologically evaluated against retinal and choroidal neovascularization as a promising drug candidate for wet agerelated macular degeneration and other neovascular eye diseases. $^{7-12}$ Moreover, we developed an enantioselective synthesis of 5,6,7-trisubstituted homoisoflavonoids such as cremastranone having the C3-stereogenic center, which was confirmed to have an R-configuration, and the antiangiogenic activity of the unnatural S-isomer was found to be more potent than the R-isomer. ${ }^{13}$ While synthetic and medicinal chemistry on 5,6,7-trisubstituted homoisoflavonoids were established by us, 5,7,8-trisubstituted homoisoflavonoids have not been well studied yet, although isolated natural products have been reported.

Naturally occurring 5,7,8-trioxygenated homoisoflavonoids have been isolated from the plants Bellevalia eigii, Drimiopsis maculata, Ledebouria graminifolia, Eucomis autumnalis, Eucomis punctata, Eucomis pallidiflora, Chionodoxa luciliae, Muscari comosum, and Dracaena cochinchinensis (Figure 2). ${ }^{14-23}$ Punctatin is known as the representative natural product for 5,7,8-trioxygenated homoisoflavonoids, and its congeners can be given a chemical name based on punctatin. B. eigii is a perennial plant belonging to the family Asparagaceae, subfamily Scilloideae. The Alali group reported the cytotoxic activity of the natural products isolated from the bulb of this plant. ${ }^{14}$ Among them, 5-hydroxy-7,8-dimethoxychroman-4-one (1), 7-O-methylpunctatin (2), 7-O-methyl-3'-hydroxypunctatin (3), 7-methyl-3,9-dihydropunctatin (4), 7-O-methyl-3'hydroxy-3,9-dihydropunctatin (5), and 7,4'-di-O-methyl-3'- hydroxy-3,9-dihydropunctatin (6) were isolated. D. maculata is distributed mainly in South Africa and belongs to the family Asparagaceae, subfamily Scilloideae. The Mulholland group isolated 7-methyl-3,9-dihydropunctatin (4) from D. maculata, which has traditionally been used as a medicine for stomach ailments in children. ${ }^{15,1615,16}$ Eucomis genus is a perennial bulb of the plant belonging to the family Asparagaceae, subfamily Scilloideae. The plants are widely distributed mainly in South Africa. The bulbs are toxic but have been used in South Africa as traditional medicines for urologic diseases, abdominal pain, and as an antipyretic. The Tamm group isolated $4^{\prime}-O$ methylpunctatin (9) from the bulb of E. autumnalis. ${ }^{17}$ Punctatin (8) and 3,9-dihydropunctatin (11) were isolated from the bulbs of E. punctata, Eucomis comosa, and E. pallidiflora. ${ }^{18,19}$ C. luciliae and M. comosum are perennial plants distributed in Southeast Europe belonging to the family Asparagaceae and subfamily Scilloideae. The Parrilli group isolated punctatin (8), 3'-hydroxypunctatin (10), 3,9-dihydropunctatin (11), and 4'-O-methyl-3,9-dihydropunctatin (12) from bulbs of plants. ${ }^{20,21} D$. cochinchinensis belongs to the family Asparagaceae and subfamily Nolinoideae in the APG IV system. L. graminifolia is one of the 16 species comprising the Ledebouria genus in Botswana. Ledebouria belongs to the family Asparagaceae, subfamily Scilloideae. The Abegza group isolated 5,7-O-dimethyl-3,9-dihydropunctatin (13) by separating the organic extract from the bulb of this plant, which has traditionally been widely used mainly for skin irritations, wound dressing, sores, coughs, backaches, gastroenteritis, and as a sedative during pregnancy. ${ }^{22}$ The Jiang group isolated dracaeconolide B (14) from the resin of this plant, which is called dragon's blood, and it has traditionally been used to treat traumatic injury, fractures, diarrhea, and dysmenorrhea. ${ }^{23}$

Although various synthetic studies of the homoisoflavonoids have been introduced, there have been few reports toward 5,7,8-trioxygenated homoisoflavonoids except Strelisky's synthesis in $1971 .^{24}$ Facile synthesis of 5,7,8-trioxygenated homoisoflavonoids has the potential to provide a general and expedient entry into a plethora of analogues for interesting biological activities. Herein, we report the first synthesis of naturally occurring and synthetic 5,7,8-trioxygenated homoisoflavonoids from the proper acetophenones, moreover, in an 
Scheme 1. Strelisky's and Our Synthetic Approaches of 5,7,8-Trioxygenated Homoisoflavonoids

(a) Strelisky's synthesis
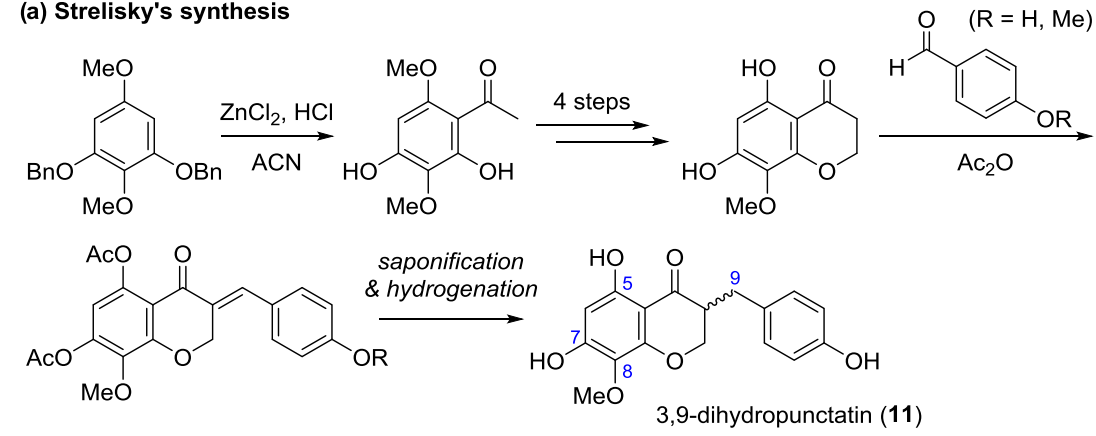

(b) Our synthetic approach

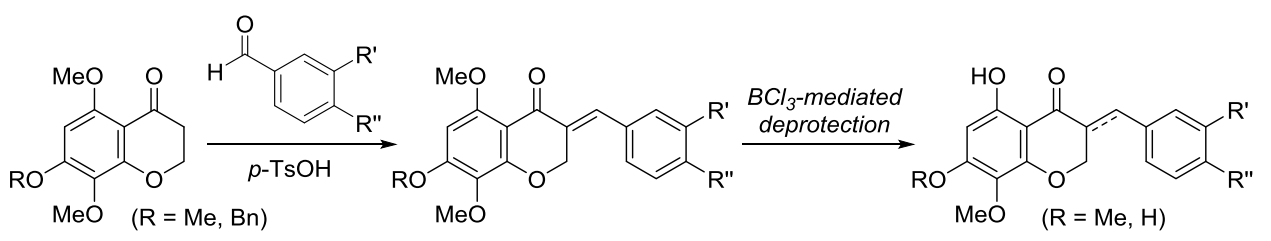

enantioselective fashion by asymmetric transfer hydrogenation and subsequent alcohol oxidation. ${ }^{13,25}$

\section{RESULTS AND DISCUSSION}

The syntheses of 5,7-dihydroxy-8-methoxychroman-4-one (7) and three homoisoflavonoids $(8,9$, and 11) were studied by Strelisky et al. ${ }^{24}$ (Scheme 1). Using the Hoesch reaction, 1,3bis(benzyloxy)-2,5-dimethoxybenzene was converted into the corresponding acetophenone, and a four-step synthesis was carried out to synthesize 7 . The acetate of 3-benzylidenechroman-4-one was obtained by a condensation reaction using $\mathrm{Ac}_{2} \mathrm{O}$, and finally natural products 8, 9, and 11 were synthesized via saponification and hydrogenation. To obtain 5,7,8-trioxygenated homoisoflavonoids with improved chemical yield and reproducibility, we contemplated aldol condensation with methoxy- and benzyl-protected chroman4-ones. Instead, mild and facile deprotection by $\mathrm{BCl}_{3}$ was carried out to synthesize various 5,7-dihydroxy-8-methoxy or 5-hydroxy-7,8-dimethoxy homoisoflavonoids. Referring to enantioselective total synthesis of cremastranone by asymmetric transfer hydrogenation, we planned to synthesize 5,7,8trioxygenated homoisoflavonoids with an R-configuration (Scheme 1).

Our synthesis of 5,7,8-trioxygenated homoisoflavonoids commenced with the synthesis of 3-benzylidenechroman-4ones having 5-hydroxy-7,8-dimethoxy groups, which were prepared from 1,2,3,5-tetramethoxybenzene 15 via 5,7,8trimethoxychroman-4-one $\mathbf{1 8}$ as shown in Scheme 2. The acetylation of 15 provided by methylation of 3,4,5-trimethoxyphenol was conducted using $\mathrm{Ac}_{2} \mathrm{O}$ and $\mathrm{BF}_{3} \cdot \mathrm{OEt}_{2}$, and 3 equiv of $\mathrm{BF}_{3} \cdot \mathrm{OEt}_{2}$ affected simultaneous demethylation in $87 \%$ yield. On the other hand, when 1 equiv of $\mathrm{BF}_{3} \cdot \mathrm{OEt}_{2}$ was used, only the acetyl group was introduced without demethylation. During the removal of the methyl group, the sterically hindered methoxy group was more easily removed among the four methoxy groups to obtain only the desired acetophenone 16. With the acetophenone 16 in hand, the $4 \mathrm{H}$-chromen-4-one 17 was provided by treatment with dimethylformamide-dimethyl acetal (DMF-DMA) and subsequent $\mathrm{c}-\mathrm{HCl}$ solution in good yield. Catalytic hydrogenation of 17 converted it to the chroman-4-one $18 . \mathrm{BCl}_{3}$-mediated demethylation afforded the naturally occurring chroman-4-one
Scheme 2. Synthesis of 3-Benzylidenechroman-4-ones Having the 5-Hydroxy-7,8-dimethoxy Group ${ }^{a}$
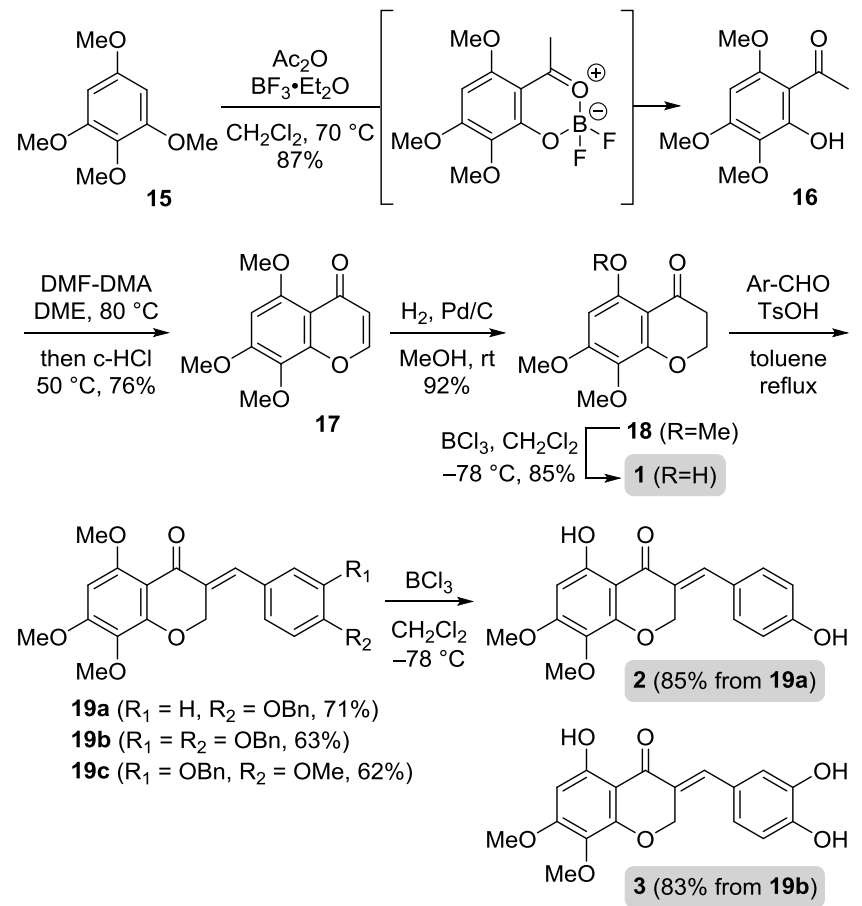

${ }^{a}$ The gray box is the reported natural product and in all other schemes.

$\mathbf{1}$ in $85 \%$ yield, and the NMR spectral data of $\mathbf{1}$ were identical to those reported. ${ }^{14}$ Three benzaldehydes, 4-benzyloxybenzaldehyde, 3-benzyloxy-4-methoxybenzaldehyde, and 3,4-bis(benzyloxy)benzaldehyde, were used for the condensation with chroman-4-one $\mathbf{1 8}$ to provide the three benzylidene compounds $(19 a-c)$ in $62-71 \%$ yield, respectively. Unfortunately, general aldol condensation did not proceed with chroman-4-one $\mathbf{1}$ having a free phenol group instead of 18 . Finally, the benzyl and methyl groups of the benzylidenechroman-4-ones $19 \mathrm{a}$ and $19 \mathrm{~b}$ under $\mathrm{BCl}_{3}$-mediated conditions were removed to provide the desired natural products $\mathbf{2}$ and 3, respectively. 
With 3-benzylidenechroman-4-ones $(19 a-c)$ in hand, four benzylchroman-4-ones, including naturally occurring homoisoflavonoid 13, were prepared by catalytic hydrogenation as shown in Scheme 3. The double bond of 3-benzylidenechro-

Scheme 3. Synthesis of 3-Benzylchroman-4-ones Having 5Hydroxy-7,8-dimethoxy Groups
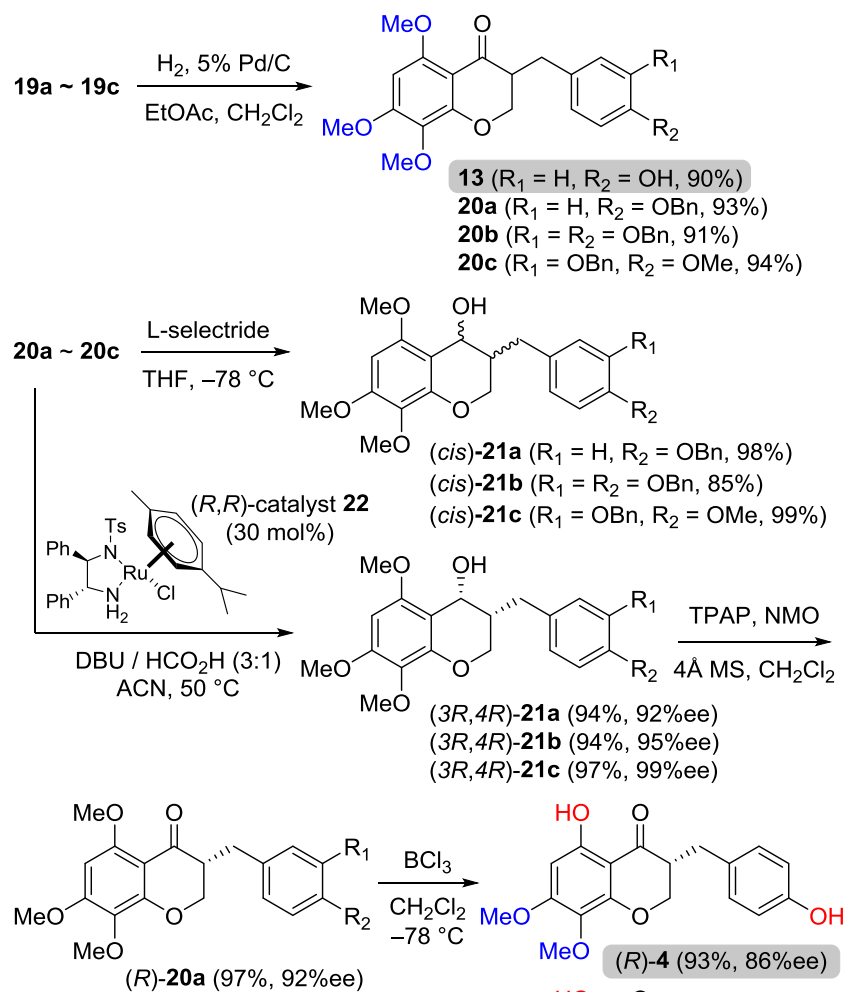
(R)-20b (93\%, 87\%ee) (R)-20c (99\%, 84\%ee)<smiles>COc1cc(O)c2c(c1OC)OC[C@H](Cc1ccc(O)c(O)c1)C2=O</smiles><smiles>COc1ccc(C[C@H]2COc3c(OC)c(OC)cc(O)c3C2=O)cc1O</smiles>

man-4-ones $(19 a-c)$ could be hydrogenated to afford 3benzylchroman-4-ones $(20 a-c)$ without the removal of benzyl groups by controlling the catalyst loading and reaction time. As in our recent report on asymmetric transfer hydrogenation by Noyori's Ru catalyst, ${ }^{26}$ the 3-benzylchroman-4-one $(20 a-c)$ was treated with $30 \mathrm{~mol} \%$ of the $(R, R)$-catalyst, 1,8diazabicyclo[5.4.0] undec-7-ene (DBU), and formic acid to afford $(3 R, 4 R)-3$-benzylchroman-4-ols $(21 \mathrm{a}-\mathrm{c})$ in excellent yields (94-97\%) and enantioselectivity (92-99\% enantiomeric excess (ee)) along with the preparation of racemic cis-3benzylchroman-4-ols by L-selectride reduction. Ley's oxidation of a secondary alcohol with tetrapropylammonium perruthenate (TPAP) and $N$-methylmorpholine $N$-oxide (NMO) was chosen as a suitable alcohol oxidation of $(3 R, 4 R)-3$ benzylchroman-4-ols $(21 \mathrm{a}-\mathrm{c})$ to afford three kinds of the corresponding $(R)$-3-benzylchroman-4-ones $((R)-20 a-c) .{ }^{27}$ Finally, $\mathrm{BCl}_{3}$-mediated deprotection was carried out to provide naturally occurring 4-6 through the cleavage of the C5-methyl in the $\mathrm{A}$ ring and benzyl group(s) in the $\mathrm{B}$ ring. ${ }^{28}$
Based on the successful synthesis of 5-hydroxy-7,8dimethoxychroman-4-one 1, we turned to consider the desired acetophenone for the synthesis of 5,7-dihydroxy-8-methoxychroman-4-one 7. Thus, the known acetophenone 23 was used as a starting material as shown in Scheme 4, and the

Scheme 4. Synthesis of 5,7-Dihydroxy-8-methoxychroman4-one

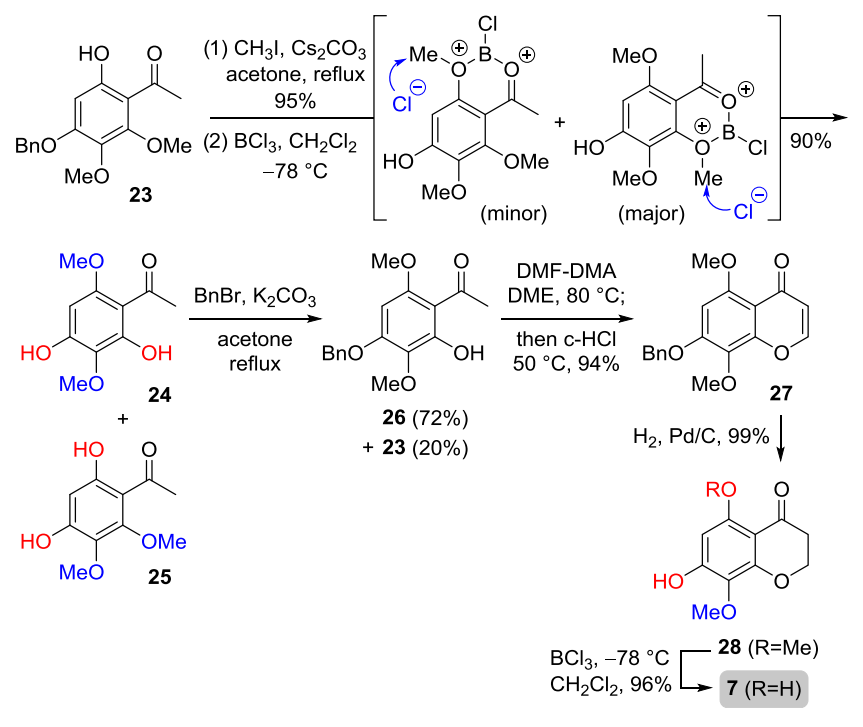

hydroxy group of $\mathbf{2 3}$ was converted into methoxy by $\mathrm{CH}_{3} \mathrm{I}$ and $\mathrm{Cs}_{2} \mathrm{CO}_{3}$, followed by selective demethylation under $\mathrm{BCl}_{3}$ mediated conditions in which it was reported to occur selectively at a hindered methyl ether in polymethoxyarenes. ${ }^{29}$ Two isomers 24 and 25 were generated by $\mathrm{BCl}_{3}$-mediated demethylation, followed by the introduction of a benzyl group on $\mathrm{C} 4$ to afford the desired acetophenones $\mathbf{2 6}$ and 23 with 72 and $20 \%$ yields, respectively. With 26 in hand, the chromen-4one 27 was afforded by the treatment of DMF-DMA and subsequent c-HCl. Finally, the conversion of 27 to the debenzylated chroman-4-one $\mathbf{2 8}$ by general catalytic hydrogenation, followed by $\mathrm{BCl}_{3}$-mediated demethylation on the $\mathrm{C5}$ position, provided the naturally occurring chroman-4-one 7 in $96 \%$ yield. $^{30}$

For the synthesis of 5,7-dihydroxy-8-methoxy homoisoflavonoids, the protection of the phenol group was necessary; otherwise, the yield of condensation was quite low. Thus, $\mathbf{2 8}$ was benzylated on the $\mathrm{C} 7$ position and condensed with four benzaldehydes to afford the corresponding 5,7,8-trioxygenated3-benzylidenechroman-4-ones $(\mathbf{2 9 a}-\mathbf{d})$ as shown in Scheme 5. $\mathrm{BCl}_{3}$-mediated deprotection was carried out to provide naturally occurring 5,7-dihydroxy-8-methoxy homoisoflavonoids $(8-10)$ in moderate yield $(42-60 \%)$. Also, 3benzylchroman-4-ones ( 30 and 31 ) were obtained by catalytic hydrogenation of $29 \mathrm{a}$ and $29 \mathrm{~d}$, and both the methyl on C5 and the benzyl on the $\mathrm{B}$ ring were smoothly removed by $\mathrm{BCl}_{3}$ mediated reactions to afford two naturally occurring 3benzylchroman-4-ones (11 and 12).

To obtain homoisoflavonoids containing either 5-hydroxy7,8-dimethoxy or 5,7-dihydroxy-8-methoxy groups, it was important to study deprotection conditions to properly remove methyl and benzyl groups from the advanced intermediates of 5,7,8-trioxygenated homoisoflavonoids such as (R)-20a-c, 29a, and 29b, as shown in Scheme 6. $\mathrm{BCl}_{3}$ was considered an excellent reagent for deprotection owing to its mild and site- 
Scheme 5. Synthesis of Homoisoflavonoids Having 5,7Dihydroxy-8-methoxy Groups

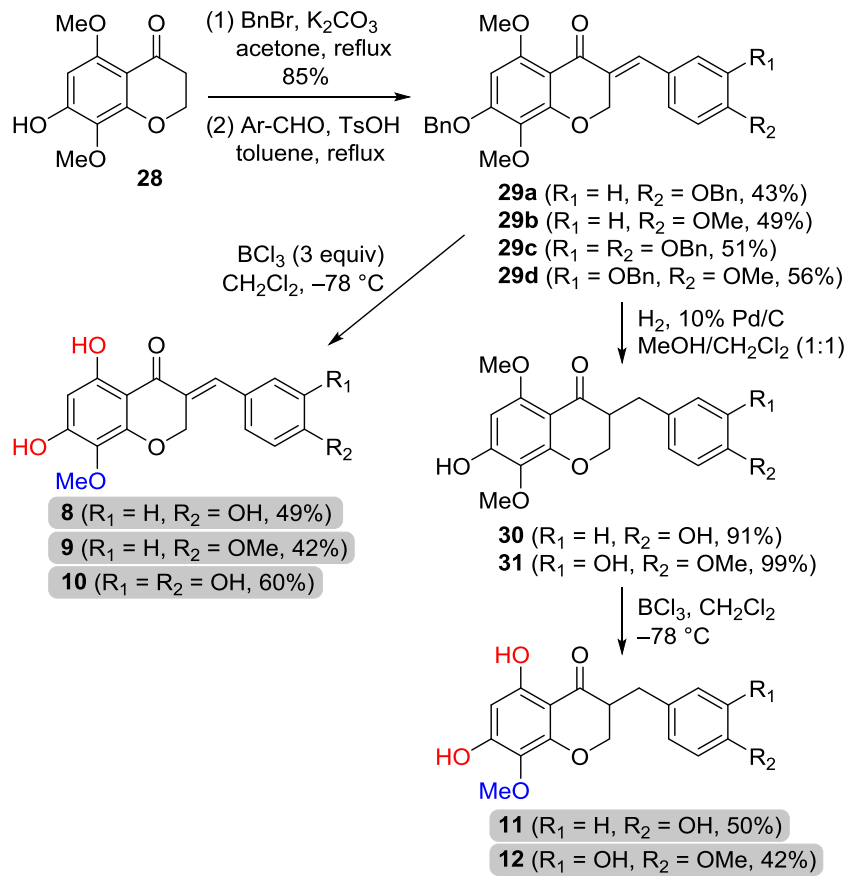

selective conditions. Interestingly, the order and process by which benzyl and methyl are removed could be understood through the structural confirmation of isolated intermediates $(32 a-c)$. In the case of phenol-deprotection of 3-benzylidenechroman-4-one (19a, 29a, and 29b), which was a highly conjugated system, the benzyl group(s) was removed first followed by methyl on the C5 position. However, slightly lower yields resulted in the overreaction of demethylation and instability of benzylidene derivatives. In 3-benzylchroman-4ones $((R)-20 \mathrm{a}$ and $(R)-20 \mathbf{b})$, on the other hand, a methyl group neighboring the carbonyl group was first removed using $\mathrm{BCl}_{3}$ to yield the resulting intermediates (33a and $33 \mathrm{~b}$ ) having a hydroxy group on the C5 position, followed by the removal of benzyl group(s) on the $\mathrm{B}$ ring finally to afford 4 and 5 , respectively. During $\mathrm{BCl}_{3}$-mediated deprotection of $(R)-20 \mathrm{c}$, the product 6 was provided via a mixture of $33 \mathrm{c}$ and $33 \mathrm{~d}$, and so it was concluded that the reactivity to removal of methyl and benzyl groups was similar.

Dracaeconolide B (14), 3-(4-hydroxybenzyl)-7-hydroxy-5,8dimethoxychromane, is unique in having the combination of a hydroxy at the $\mathrm{C} 7$ position and two methoxys on $\mathrm{C} 5$ and $\mathrm{C} 8$ positions of the homoisoflavonoid and a natural chromane skeleton, deoxygenated chroman-4-one, compared to other 5,7,8-trioxygenated homoisoflavonoids. We undertook catalytic hydrogenation of the previous intermediate 29a to reduce the double bond and cleave two benzyl groups as shown in Scheme 7. Unfortunately, the spectral data of our synthetic compound 14 were not identical to the reported ones of ${ }^{1} \mathrm{H}$ and ${ }^{13} \mathrm{C}$ NMR spectroscopies. ${ }^{23}$ We attempted to synthesize other regioisomers of dracaeconolide $\mathrm{B}$ to determine whether its structure would be corrected. 3-Benzylidenechroman-4-one 19a was converted to 5-hydroxy-7,8-dimethoxy isomer 34 by $\mathrm{BCl}_{3}$ reaction and catalytic hydrogenation of 2 . In contrast, 19a was reduced to 3-(4-hydroxybenzyl)-5,7,8-trimethoxychromane 35 in which the sterically hindered C8-methoxy group could be cleaved predominantly under the $\mathrm{BCl}_{3}$-mediated condition to provide the 8-hydroxy-5,7-dimethoxy isomer 36 . The structural elucidation of three different regioisomers (14, 34, and 36) was confirmed by comparison of the ${ }^{1} \mathrm{H}$ and ${ }^{13} \mathrm{C}$ NMR data (Table S14) and two-dimensional (2D) NMR spectroscopy (Figures S1-S3). The position and combination of one hydroxy and two methoxy groups on the C5, C7, and $\mathrm{C} 8$ positions of the $\mathrm{A}$ ring were assigned by heteronuclear multiple bond correlation (HMBC) correlations from $\mathrm{H}-6$ to C-4a, C-5, C-7, and C-8; $\mathrm{H}_{2}-4$ to C-4a, C-5, C-6, C-8a, and C8 ; and $\mathrm{H}_{2}-2$ to $\mathrm{C}-2$ and $\mathrm{C}-8 \mathrm{a}$.

\section{CONCLUSIONS}

We have developed total syntheses of 2 naturally occurring 5,7,8-trioxygenated chroman-4-ones and 12 5,7,8-trioxygenated homoisoflavonoids, including 10 synthesized for the first time. 1,3,4,5-Tetramethoxybenzene and 4'-benzyloxy-2',3'dimethoxy- $6^{\prime}$-hydroxyacetophenone were used as starting materials. Asymmetric transfer hydrogenation using Noyori's $\mathrm{Ru}$ catalyst provided three 5,7,8-trioxygenated homoisoflavanones having an R-configuration in $>94 \%$ yield and $>92 \%$ ee. During $\mathrm{BCl}_{3}$-mediated deprotection, we observed that the order in which the protecting groups are removed differs depending on the substrate. To synthesize the 5,7,8trioxygenated benzylchromane dracaeconolide $\mathrm{B}$, the synthesis and structural analysis of three regioisomers were executed. Our research on 5,7,8-trioxygenated homoisoflavonoids will be useful for the synthesis of related natural products and drug discovery.

\section{EXPERIMENTAL SECTION}

All starting materials and reagents were obtained from commercial sources and used without further purification. Air- and moisture-sensitive reactions were performed under nitrogen. Flash column chromatography was performed using silica gel $60(230-400$ mesh, Merck) with the indicated solvents. Thin-layer chromatography (TLC) was performed using $0.25 \mathrm{~mm}$ silica gel plates (Merck). ${ }^{1} \mathrm{H}$ and ${ }^{13} \mathrm{C}\left\{{ }^{1} \mathrm{H}\right\}$ NMR spectra were recorded on a Bruker $600 \mathrm{MHz}$ spectrometer as solutions in deuterochloroform $\left(\mathrm{CDCl}_{3}\right)$ or methanol- $d_{4}\left(\mathrm{CD}_{3} \mathrm{OD}\right) .{ }^{1} \mathrm{H}$ NMR data were reported on the order of chemical shift, multiplicity ( $s$, singlet; $d$, doublet; $t$, triplet; q, quartet; m, multiplet and/or multiplet resonances), number of protons, and coupling constant ( $J$ value) in hertz $(\mathrm{Hz})$. Enantiomeric excesses were determined by highperformance liquid chromatography (HPLC) on an Agilent 1100 using one chiral column (CHIRALPAK IA, IB, IC, ID, IG). High-resolution mass spectra (HRMS) were recorded on an Agilent 6530 quadrupole time-of-flight (Q-TOF) liquid chromatography (LC)/tandem mass spectrometry (MS/MS) system (electrospray ionization (ESI)).

1,2,3,5-Tetramethoxybenzene (15). To an N,N-dimethylformamide $(20 \mathrm{~mL})$ solution of 3,4,5-trimethoxy-phenol $(3.0 \mathrm{~g}, 16 \mathrm{mmol})$, dimethyl sulfate $(4.6 \mathrm{~mL}, 49 \mathrm{mmol})$ and $\mathrm{Cs}_{2} \mathrm{CO}_{3}(10 \mathrm{~g}, 33 \mathrm{mmol})$ were added. The reaction mixture was refluxed for $4 \mathrm{~h}$. After cooling to ambient temperature, the reaction mixture was diluted with ethyl acetate and the organic phase was washed with water, dried over anhydrous $\mathrm{Na}_{2} \mathrm{SO}_{4}$, and concentrated under reduced pressure. The residue was purified by flash column chromatography on silica gel (ethyl acetate $/ n$-hexane $=1: 5)$ to afford 1,2,3,5-tetramethoxybenzene (15) $(3.2 \mathrm{~g}, 98 \%) .{ }^{1} \mathrm{H}$ NMR $\left(600 \mathrm{MHz}, \mathrm{CDCl}_{3}\right) \delta 6.15(\mathrm{~s}$, $2 \mathrm{H}), 3.84(\mathrm{~s}, 6 \mathrm{H}), 3.78(\mathrm{~s}, 3 \mathrm{H}), 3.78(\mathrm{~s}, 3 \mathrm{H}) .{ }^{13} \mathrm{C}\left\{{ }^{1} \mathrm{H}\right\} \mathrm{NMR}$ 
Scheme 6. Sequential $\mathrm{BCl}_{3}$-Mediated Deprotection of (a) 3-Benzylidenechroman-4-one and (b) 3-Benzylchroman-4-one

(a) $\mathrm{BCl}_{3}$ reaction of 3-benzylidenechroman-4-ones

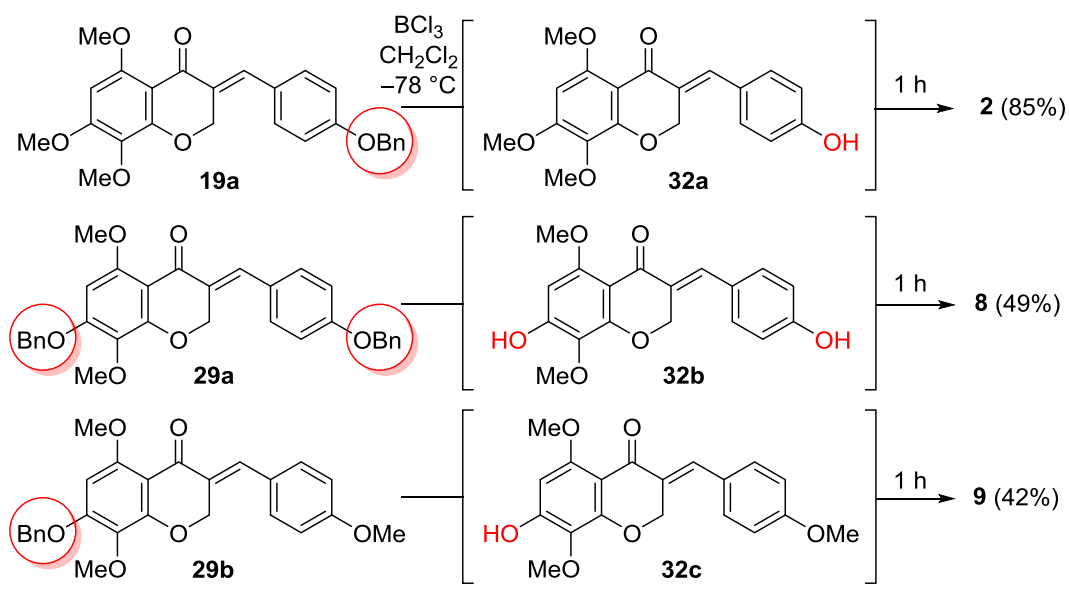

(b) $\mathrm{BCl}_{3}$ reaction of 3-benzylchroman-4-ones
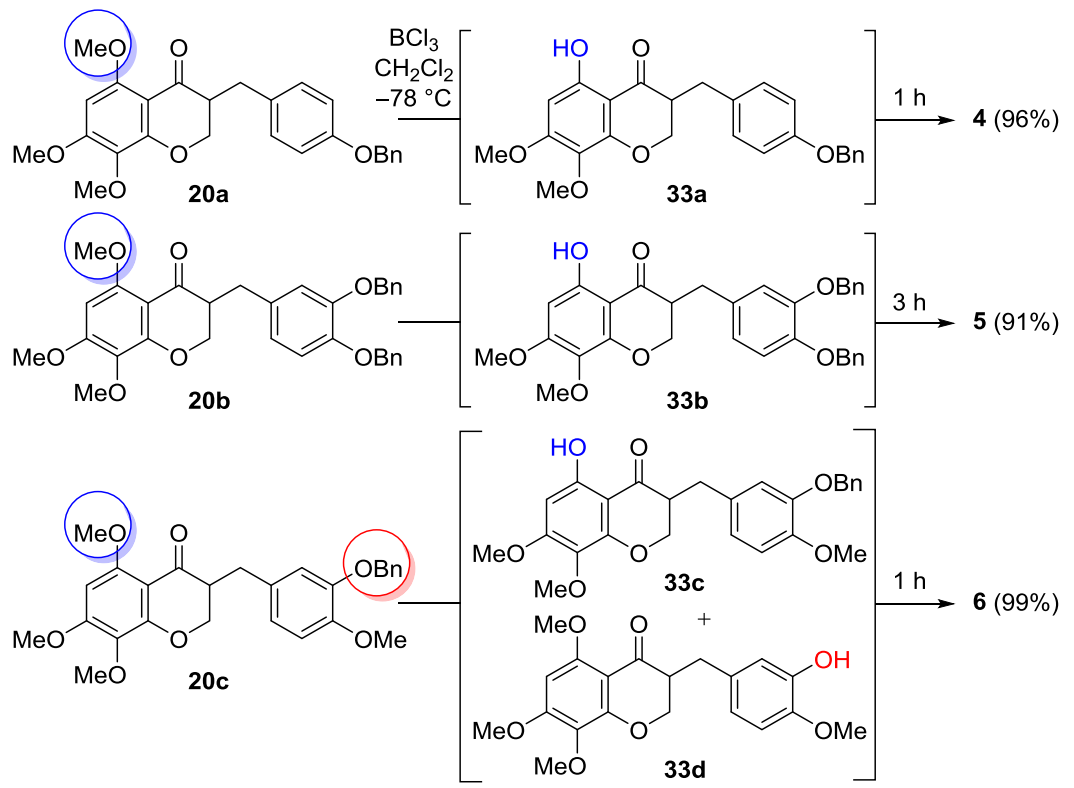

Scheme 7. Synthesis of Suggested Dracaeconolide B and Its Two Isomers
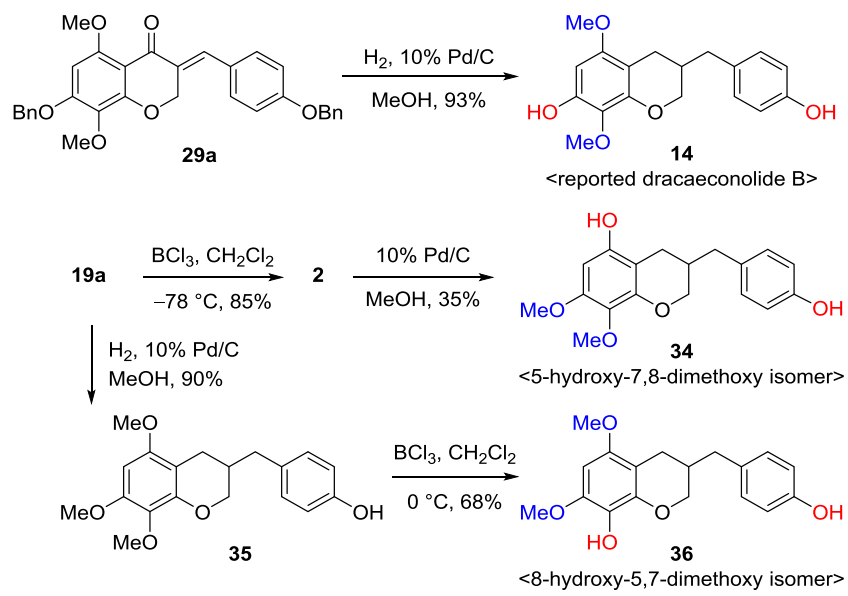

$\left(150 \mathrm{MHz}, \mathrm{CDCl}_{3}\right) \delta 156.4,153.8,132.4,91.7,61.1,56.2$, 55.6. HRMS (ESI) $m / z:[\mathrm{M}+\mathrm{H}]^{+}$calcd for $\mathrm{C}_{10} \mathrm{H}_{14} \mathrm{O}_{4}$ 199.0970; found, 199.0966.

1-(2-Hydroxy-3,4,6-trimethoxyphenyl)ethan-1-one (16). To a chloroform (15 mL) solution of 1,2,3,5tetramethoxybenzene (15) ( $3.2 \mathrm{~g}, 16 \mathrm{mmol})$, acetic anhydride $(1.7 \mathrm{~mL}, 18 \mathrm{mmol})$ and $\mathrm{BF}_{3} \cdot \mathrm{OEt}_{2}(6.9 \mathrm{~mL}, 56 \mathrm{mmol})$ were added at $0{ }^{\circ} \mathrm{C}$. After stirring at $70{ }^{\circ} \mathrm{C}$ for $4 \mathrm{~h}$, the reaction mixture was cooled, and ice-cold water and $5 \% \mathrm{NaOH}(5 \mathrm{~mL})$ were poured into the mixture. The reaction mixture was diluted with ethyl acetate, and the organic phase was washed with water and brine, dried over anhydrous $\mathrm{Na}_{2} \mathrm{SO}_{4}$, and concentrated under reduced pressure. The residue was purified by flash column chromatography (ethyl acetate $/ n$-hexane $=$ 1:3) to afford 1-(2-hydroxy-3,4,6-trimethoxyphenyl)ethan-1one (16) (3.1 g, 87\%). ${ }^{1} \mathrm{H}$ NMR (600 MHz, $\left.\mathrm{CDCl}_{3}\right) \delta 13.80$ (s, 1H), 5.95 (s, 1H), $3.93(\mathrm{~s}, 3 \mathrm{H}), 3.88(\mathrm{~s}, 3 \mathrm{H}), 3.80(\mathrm{~s}, 3 \mathrm{H})$, $2.61(\mathrm{~s}, 3 \mathrm{H}) \cdot{ }^{13} \mathrm{C}\left\{{ }^{1} \mathrm{H}\right\}$ NMR $\left(150 \mathrm{MHz}, \mathrm{CDCl}_{3}\right) \delta 203.9$, $159.2,158.9,158.5,130.6,106.4,86.5,60.8,56.1,55.7,33.3$ 
HRMS (ESI) $m / z$ : $[\mathrm{M}+\mathrm{H}]^{+}$calcd for $\mathrm{C}_{11} \mathrm{H}_{14} \mathrm{O}_{5}$ 227.0919; found, 227.0914 .

5,7,8-Trimethoxy-4H-chromen-4-one (17). To a solution of 1-(2-hydroxy-3,4,6-trimethoxyphenyl)ethan-1-one (16) $(2.7 \mathrm{~g}, 12 \mathrm{mmol})$ in dimethoxyethane (DME) $(30 \mathrm{~mL})$ was added $N, N$-dimethylformamide dimethyl acetal $(4.8 \mathrm{~mL}$, $36 \mathrm{mmol}$ ). After stirring for $24 \mathrm{~h}$ at $80{ }^{\circ} \mathrm{C}$, the reaction mixture was cooled to $0{ }^{\circ} \mathrm{C}$ and $\mathrm{c}-\mathrm{HCl}(6 \mathrm{~mL})$ was added. After stirring for $1 \mathrm{~h}$ at $50{ }^{\circ} \mathrm{C}$, the reaction mixture was diluted with ethyl acetate and the organic phase was washed with water and brine and dried over anhydrous $\mathrm{Na}_{2} \mathrm{SO}_{4}$. The solvent was removed under reduced pressure and purified by flash column chromatography on silica gel (ethyl acetate/ $n$-hexane/methanol $=1: 1: 0.1)$ to afford 5,7,8-trimethoxy-4H-chromen-4-one (17) $(2.2 \mathrm{~g}, 76 \%) .{ }^{1} \mathrm{H}$ NMR $\left(600 \mathrm{MHz}, \mathrm{CDCl}_{3}\right) \delta 7.70(\mathrm{~d}$, $1 \mathrm{H}, J=5.9 \mathrm{~Hz}), 6.43(\mathrm{~s}, 1 \mathrm{H}), 6.18(\mathrm{~d}, 1 \mathrm{H}, J=5.9 \mathrm{~Hz}), 3.99(\mathrm{~s}$, $3 \mathrm{H}), 3.96(\mathrm{~s}, 3 \mathrm{H}), 3.88(\mathrm{~s}, 3 \mathrm{H}) .{ }^{13} \mathrm{C}\left\{{ }^{1} \mathrm{H}\right\}$ NMR $(150 \mathrm{MHz}$, $\left.\mathrm{CDCl}_{3}\right) \delta 176.9,156.7,156.6,152.9,152.4,130.7,114.2$, 110.2 , 92.7, 61.8, 56.7, 56.4. HRMS (ESI) $m / z:[\mathrm{M}+\mathrm{H}]^{+}$ calcd for $\mathrm{C}_{12} \mathrm{H}_{12} \mathrm{O}_{5}$ 237.0763; found, 237.0760.

5,7,8-Trimethoxychroman-4-one (18). 5,7,8-Trimethoxy-4H-chromen-4-one (17) (2.8 g, $12 \mathrm{mmol})$ and $10 \%$ $\mathrm{Pd} / \mathrm{C}(0.38 \mathrm{~g}, 3.5 \mathrm{mmol})$ in methanol $(40 \mathrm{~mL})$ were placed under an atmosphere of hydrogen. After stirring for $1 \mathrm{~h}$, the mixture was filtered through a Celite pad. After the filtrate was concentrated under reduced pressure, purification of the residue via flash column chromatography on silica gel (ethyl acetate $/ n$-hexane $/$ methanol $=1: 1: 0.1)$ afforded 5,7,8-trimethoxychroman-4-one (18) (2.6 g, 92.\%). ${ }^{1} \mathrm{H}$ NMR (600 MHz, $\left.\mathrm{CDCl}_{3}\right) \delta 6.09(\mathrm{~s}, 1 \mathrm{H}), 4.50(\mathrm{t}, 2 \mathrm{H}, J=6.4 \mathrm{~Hz}), 3.93(\mathrm{~s}, 3 \mathrm{H})$, $3.90(\mathrm{~s}, 3 \mathrm{H}), 3.79(\mathrm{~s}, 3 \mathrm{H}), 2.72(\mathrm{t}, 2 \mathrm{H}, J=6.4 \mathrm{~Hz}) .{ }^{13} \mathrm{C}\left\{{ }^{1} \mathrm{H}\right\}$ NMR $\left(150 \mathrm{MHz}, \mathrm{CDCl}_{3}\right) \delta 189.5,158.6,158.1,156.7,130.8$, 106.7, 89.3, 67.3, 61.3, 56.3, 56.1, 39.0. HRMS (ESI) $\mathrm{m} / z$ : [M $+\mathrm{H}]^{+}$calcd for $\mathrm{C}_{12} \mathrm{H}_{14} \mathrm{O}_{5}$ 239.0919; found, 239.0912 .

5-Hydroxy-7,8-dimethoxychroman-4-one (1). To a solution of 5,7,8-trimethoxychroman-4-one (18) $(50 \mathrm{mg}$, $0.21 \mathrm{mmol})$ in $\mathrm{CH}_{2} \mathrm{Cl}_{2}(3 \mathrm{~mL})$ was added boron trichloride $\left(0.62 \mathrm{~mL}, 1.0 \mathrm{M}\right.$ solution in $\mathrm{CH}_{2} \mathrm{Cl}_{2}$ ) at $-78{ }^{\circ} \mathrm{C}$. After stirring for $1 \mathrm{~h}$, the reaction mixture was diluted with $\mathrm{CH}_{2} \mathrm{Cl}_{2}$ and washed with water, dried over anhydrous $\mathrm{Na}_{2} \mathrm{SO}_{4}$, and concentrated under reduced pressure. The residue was purified by flash column chromatography on silica gel (ethyl acetate $/ n$ hexane $=1: 3)$ to afford 5-hydroxy-7,8-dimethoxychroman-4one (1) (40 mg, 85\%). ${ }^{1} \mathrm{H}$ NMR (600 MHz, $\left.\mathrm{CDCl}_{3}\right) \delta 11.98$ $(\mathrm{s}, 1 \mathrm{H}), 6.08(\mathrm{~s}, 1 \mathrm{H}), 4.52(\mathrm{t}, 2 \mathrm{H}, J=6.3 \mathrm{~Hz}), 3.88(\mathrm{~s}, 2 \mathrm{H})$, $3.77(\mathrm{~s}, 2 \mathrm{H}), 2.79(\mathrm{t}, 2 \mathrm{H}, J=6.3 \mathrm{~Hz}) \cdot{ }^{13} \mathrm{C}\left\{{ }^{1} \mathrm{H}\right\} \operatorname{NMR}(150$ $\left.\mathrm{MHz} \mathrm{CDCl}_{3}\right) \delta 196.2,161.5,160.3,154.0,129.6,103.3,93.1$, 67.1, 61.5, 56.4, 36.8. HRMS (ESI) $m / z:[\mathrm{M}+\mathrm{H}]^{+}$calcd for $\mathrm{C}_{11} \mathrm{H}_{12} \mathrm{O}_{5}$ 225.0763; found, 225.0760 .

(E)-3-(4' -(Benzyloxy)benzylidene)-5,7,8-trimethoxychroman-4-one (19a). To a solution of 5,7,8-trimethoxychroman-4-one (18) (0.50 g, $2.1 \mathrm{mmol})$ in toluene $(20 \mathrm{~mL})$ were added 4-benzyloxybenzaldehyde $(0.67 \mathrm{~g}, 3.1 \mathrm{mmol})$ and p-toluenesulfonic acid (36 mg, $0.21 \mathrm{mmol}$ ) at $0{ }^{\circ} \mathrm{C}$. The reaction mixture was refluxed for $12 \mathrm{~h}$. After cooling to ambient temperature, the mixture was concentrated under reduced pressure. The residue was purified by flash column chromatography on silica gel (ethyl acetate $/ n$-hexane $/$ methanol $=1: 2: 0.1)$ to afford $(E)-3-\left(4^{\prime}\right.$-(benzyloxy)benzylidene $)$ 5,7,8-trimethoxychroman-4-one (19a) (0.64 g, 71\%). ${ }^{1} \mathrm{H}$ NMR $\left(600 \mathrm{MHz}, \mathrm{CDCl}_{3}\right) \delta 7.69(\mathrm{~s}, 1 \mathrm{H}), 7.43(\mathrm{~d}, J=7.4 \mathrm{~Hz}, 2 \mathrm{H})$, $7.37(\mathrm{t}, 2 \mathrm{H}, J=7.6 \mathrm{~Hz}), 7.30(\mathrm{t}, 1 \mathrm{H}, J=7.3 \mathrm{~Hz}), 6.92(\mathrm{~d}, 1 \mathrm{H}, J$ $=8.4 \mathrm{~Hz}), 6.88(\mathrm{dd}, 1 \mathrm{H}, J=8.4,1.7 \mathrm{~Hz}), 6.78(\mathrm{~d}, 1 \mathrm{H}, J=1.7$
$\mathrm{Hz}), 6.15$ (s, 1H), 5.17 (s, 2H), 5.13 (d, 2H, $J=1.5 \mathrm{~Hz}), 3.93$ (s, 3H), $3.92(\mathrm{~s}, 3 \mathrm{H}), 3.92(\mathrm{~s}, 3 \mathrm{H}), 3.78(\mathrm{~s}, 3 \mathrm{H}) \cdot{ }^{13} \mathrm{C}\left\{{ }^{1} \mathrm{H}\right\}$ NMR $\left(150 \mathrm{MHz}, \mathrm{CDCl}_{3}\right) \delta 179.8,159.6,158.4,158.3,155.9$, $136.5,135.9,131.8,130.9,130.1,128.7,128.1,127.6,127.5$, 115.0, 107.6, 90.1, 70.1, 67.9, 61.3, 56.3, 56.1. HRMS (ESI) $\mathrm{m} /$ $z:[\mathrm{M}+\mathrm{H}]^{+}$calcd for $\mathrm{C}_{26} \mathrm{H}_{24} \mathrm{O}_{6} 433.1651$; found, 433.1650 .

(E)-3-(3',4'-Bis(benzyloxy)benzylidene)-5,7,8-trimethoxychroman-4-one (19b). To a solution of 5,7,8trimethoxychroman-4-one $(\mathbf{1 8})(0.50 \mathrm{~g}, 2.1 \mathrm{mmol})$ in toluene $(20 \mathrm{~mL})$ were added 3,4-bis(benzyloxy)benzaldehyde $(1.0 \mathrm{~g}$, $3.2 \mathrm{mmol})$ and $p$-toluenesulfonic acid $(36 \mathrm{mg}, 0.21 \mathrm{mmol})$ at 0

${ }^{\circ} \mathrm{C}$. The reaction mixture was refluxed for $12 \mathrm{~h}$. After cooling to ambient temperature, the mixture was concentrated under reduced pressure. The residue was purified by flash column chromatography on silica gel (ethyl acetate $/ \mathrm{CH}_{2} \mathrm{Cl}_{2}=1: 5$ ) to afford (E)-3-(3',4'-bis(benzyloxy)benzylidene)-5,7,8-trimethoxychroman-4-one (19b) (0.71 g, 63\%). ${ }^{1} \mathrm{H}$ NMR (600 $\left.\mathrm{MHz} \mathrm{CDCl}_{3}\right) \delta 7.70(\mathrm{~s}, 1 \mathrm{H}), 7.47-7.43(\mathrm{~m}, 4 \mathrm{H}), 7.38(\mathrm{td}$, $4 \mathrm{H}, J=7.5,2.1 \mathrm{~Hz}), 7.34-7.30(\mathrm{~m}, 2 \mathrm{H}), 6.97-6.94(\mathrm{~m}, 1 \mathrm{H})$, $6.84(\mathrm{dd}, 2 \mathrm{H}, J=5.4,1.9 \mathrm{~Hz}), 6.17(\mathrm{~s}, 1 \mathrm{H}), 5.22(\mathrm{~s}, 2 \mathrm{H}), 5.19$ (s, $2 \mathrm{H}), 5.15(\mathrm{~d}, 2 \mathrm{H}, J=1.7 \mathrm{~Hz}), 3.94(\mathrm{~s}, 3 \mathrm{H}), 3.94(\mathrm{~s}, 3 \mathrm{H})$, $3.79(\mathrm{~s}, 3 \mathrm{H}) \cdot{ }^{13} \mathrm{C}\left\{{ }^{1} \mathrm{H}\right\}$ NMR $\left(150 \mathrm{MHz}, \mathrm{CDCl}_{3}\right) \delta 179.9$, 158.5 , 158.5, 156.0, 150.1, 148.6, 137.0, 136.9, 136.1, 131.1, $130.4,128.8,128.7,128.2,128.1,127.4,127.4,124.3,116.9$, 114.4, 107.8, 90.2, 71.6, 71.2, 67.9, 61.5, 56.5, 56.2. HRMS (ESI) $m / z:[\mathrm{M}+\mathrm{H}]^{+}$calcd for $\mathrm{C}_{33} \mathrm{H}_{30} \mathrm{O}_{7}$ 539.2070; found, 539.2067.

(E)-3-(3'-(Benzyloxy)-4'-methoxybenzylidene)-5,7,8trimethoxychroman-4-one (19c). To a solution of 5,7,8trimethoxychroman-4-one $(\mathbf{1 8})(0.50 \mathrm{~g}, 2.1 \mathrm{mmol})$ in toluene $(20 \mathrm{~mL})$ were added 3-(benzyloxy)-4-methoxybenzaldehyde $(0.77 \mathrm{~g}, 3.2 \mathrm{mmol})$ and $p$-toluenesulfonic acid $(36 \mathrm{mg}, 0.21$ $\mathrm{mmol}$ ) at $0{ }^{\circ} \mathrm{C}$. The reaction mixture was refluxed for $13 \mathrm{~h}$. After cooling to ambient temperature, the mixture was concentrated under reduced pressure. The residue was purified by flash column chromatography on silica gel (ethyl acetate/ $n$ hexane/methanol = 1:2:0.1) to afford $(E)-3$-( $3^{\prime}$-(benzyloxy)$4^{\prime}$-methoxybenzylidene)-5,7,8-trimethoxychroman-4-one (19c) $(0.60 \mathrm{~g}, 62 \%) .{ }^{1} \mathrm{H} \mathrm{NMR}\left(600 \mathrm{MHz}, \mathrm{CDCl}_{3}\right) \delta 7.77(\mathrm{~s}$, $1 \mathrm{H}), 7.41(\mathrm{~d}, 2 \mathrm{H}, J=7.3 \mathrm{~Hz}), 7.37(\mathrm{t}, 2 \mathrm{H}, J=7.3 \mathrm{~Hz}), 7.32(\mathrm{~d}$, $1 \mathrm{H}, J=7.3 \mathrm{~Hz}), 7.23(\mathrm{~d}, 2 \mathrm{H}, J=8.7 \mathrm{~Hz}), 7.00(\mathrm{~d}, 2 \mathrm{H}, J=8.7$ $\mathrm{Hz}), 6.16(\mathrm{~s}, 1 \mathrm{H}), 5.28(\mathrm{~d}, 2 \mathrm{H}, J=1.6 \mathrm{~Hz}), 5.08(\mathrm{~s}, 2 \mathrm{H}), 3.92$ (s, 3H), 3.91 (s, 3H), 3.77 (s, 3H). ${ }^{13} \mathrm{C}\left\{{ }^{1} \mathrm{H}\right\}$ NMR $(150 \mathrm{MHz}$, $\left.\mathrm{CDCl}_{3}\right) \delta 179.8,158.4,158.4,155.9,150.8,147.9,136.8$, $136.1,131.0,130.2,128.8,128.1,127.5,127.3,124.2,115.9$, 111.6, 107.7, 90.2, 71.2, 67.9, 61.4, 56.4, 56.1, 56.1. HRMS (ESI) $m / z:[\mathrm{M}+\mathrm{H}]^{+}$calcd for $\mathrm{C}_{27} \mathrm{H}_{26} \mathrm{O}_{7}$ 463.1757; found, 463.1754 .

7-O-Methoxypunctatin (2). To a solution of (E)-3-(4'(benzyloxy)benzylidene)-5,7,8-trimethoxychroman-4-one (19a) $(31 \mathrm{mg}, 0.07 \mathrm{mmol})$ in $\mathrm{CH}_{2} \mathrm{Cl}_{2}(2 \mathrm{~mL})$ was added boron trichloride $\left(0.21 \mathrm{~mL}, 1.0 \mathrm{M}\right.$ solution in $\left.\mathrm{CH}_{2} \mathrm{Cl}_{2}\right)$ at -78 ${ }^{\circ} \mathrm{C}$. After stirring for $1 \mathrm{~h}$, the reaction mixture was diluted with $\mathrm{CH}_{2} \mathrm{Cl}_{2}$, washed with water, dried over anhydrous $\mathrm{Na}_{2} \mathrm{SO}_{4}$, and concentrated under reduced pressure. The residue was purified by flash column chromatography on silica gel (ethyl acetate $/ n$-hexane $/$ methanol $=1: 1: 0.1)$ to afford 7-O-methoxypunctatin (2) $(20 \mathrm{mg}, 85 \%) .{ }^{1} \mathrm{H}$ NMR $\left(600 \mathrm{MHz}, \mathrm{CDCl}_{3}\right) \delta$ $12.66(\mathrm{~s}, 1 \mathrm{H}), 7.79(\mathrm{~s}, 1 \mathrm{H}), 7.20(\mathrm{~d}, 2 \mathrm{H}, \mathrm{J}=8.6 \mathrm{~Hz}), 6.91(\mathrm{~d}$, $2 \mathrm{H}, J=8.6 \mathrm{~Hz}), 6.11(\mathrm{~s}, 1 \mathrm{H}), 5.55(\mathrm{~s}, 1 \mathrm{H}), 5.34(\mathrm{~d}, 2 \mathrm{H}, J=1.7$ $\mathrm{Hz}), 3.88$ (s, 3H), $3.76(\mathrm{~s}, 3 \mathrm{H}) .{ }^{13} \mathrm{C}\left\{{ }^{1} \mathrm{H}\right\}$ NMR $(150 \mathrm{MHz}$, $\left.\mathrm{CDCl}_{3}\right) \delta 185.8,161.5,161.1,157.5,153.0,137.7,132.5$, $129.5,127.6,127.0,116.1,103.2$, 93.4, 67.8, 61.6, 56.4. HRMS 
(ESI) $m / z:[\mathrm{M}+\mathrm{H}]^{+}$calcd for $\mathrm{C}_{18} \mathrm{H}_{16} \mathrm{O}_{6}$ 329.1025; found, 329.1023 .

7-O-Methyl-3'-hydroxypunctatin (3). To a solution of (E)-3-(3',4'-bis(benzyloxy)benzylidene)-5,7,8-trimethoxychroman-4-one (19b) (31 mg, $0.06 \mathrm{mmol})$ in $\mathrm{CH}_{2} \mathrm{Cl}_{2}(2 \mathrm{~mL})$ was added boron trichloride $(0.17 \mathrm{~mL}, 1.0 \mathrm{M}$ solution in $\mathrm{CH}_{2} \mathrm{Cl}_{2}$ ) at $0{ }^{\circ} \mathrm{C}$. After stirring for $30 \mathrm{~min}$, the reaction mixture was diluted with $\mathrm{CH}_{2} \mathrm{Cl}_{2}$, washed with water, dried over $\mathrm{Na}_{2} \mathrm{SO}_{4}$, and concentrated under reduced pressure. The residue was purified by flash column chromatography on silica gel (ethyl acetate $/ n$-hexane/methanol $=1: 1: 0.1$ ) to afford $(E)$ 3-(3,4-dihydroxybenzylidene)-5-hydroxy-7,8-dimethoxychroman-4-one (3) (16 mg, 83\%). ${ }^{1} \mathrm{H}$ NMR (600 MHz, $\left.\mathrm{CD}_{3} \mathrm{OD}\right)$ $\delta 7.72(\mathrm{~s}, 1 \mathrm{H}), 6.89(\mathrm{~d}, 1 \mathrm{H}, J=8.2 \mathrm{~Hz}), 6.85(\mathrm{~s}, 1 \mathrm{H}), 6.81(\mathrm{~d}$, $1 \mathrm{H}, J=8.2 \mathrm{~Hz}), 6.19(\mathrm{~s}, 1 \mathrm{H}), 5.40(\mathrm{~s}, 1 \mathrm{H}), 3.89(\mathrm{~s}, 1 \mathrm{H}), 3.72$ $(\mathrm{s}, 1 \mathrm{H}) .{ }^{13} \mathrm{C}\left\{{ }^{1} \mathrm{H}\right\}$ NMR $\left(150 \mathrm{MHz}, \mathrm{CD}_{3} \mathrm{OD}\right) \delta 187.1,162.9$, $162.2,154.5,149.4,146.8,139.3,130.7,128.0,127.5,125.1$, $118.6,116.8,104.1,94.2,69.1,61.7,56.9$. HRMS (ESI) $\mathrm{m} / z$ : $[\mathrm{M}+\mathrm{H}]^{+}$calcd for $\mathrm{C}_{18} \mathrm{H}_{16} \mathrm{O}_{7}$ 345.0974; found, 345.0978.

5,7,8-Trimethoxy-3-(4' -hydroxybenzyl)-4-chromanone (13). (E)-3-(4'-(Benzyloxy)benzylidene)-5,7,8-trimethoxychroman-4-one (19a) (30 $\mathrm{mg}, 0.07 \mathrm{mmol}$ ) and $10 \%$ $\mathrm{Pd} / \mathrm{C}(3.7 \mathrm{mg}, 0.03 \mathrm{mmol})$ in methanol $(3 \mathrm{~mL})$ were placed under an atmosphere of hydrogen. After stirring for $2 \mathrm{~h}$, the mixture was filtered through a Celite pad. After the filtrate was concentrated under reduced pressure, purification of the residue via flash column chromatography on silica gel (ethyl acetate $/ n$-hexane $/$ methanol $=1: 2: 0.1)$ afforded 5,7,8-trimethoxy-3-(4'-hydroxybenzyl)-4-chromanone (13) (22 mg, $90 \%) .{ }^{1} \mathrm{H}$ NMR $\left(600 \mathrm{MHz}, \mathrm{CD}_{3} \mathrm{OD}\right) \delta 7.08(\mathrm{~d}, 2 \mathrm{H}, J=8.1$ $\mathrm{Hz}), 6.76(\mathrm{~d}, 2 \mathrm{H}, J=8.1 \mathrm{~Hz}), 6.32(\mathrm{~s}, 1 \mathrm{H}), 4.34(\mathrm{dd}, 1 \mathrm{H}, J=$ $11.3,3.8 \mathrm{~Hz}), 4.18(\mathrm{dd}, 1 \mathrm{H}, J=11.3,7.1 \mathrm{~Hz}), 3.96(\mathrm{~s}, 3 \mathrm{H})$, $3.90(\mathrm{~s}, 3 \mathrm{H}), 3.73(\mathrm{~s}, 3 \mathrm{H}), 3.06(\mathrm{dd}, J=13.8,4.6 \mathrm{~Hz}, 1 \mathrm{H})$, $2.76-2.70(\mathrm{~m}, 1 \mathrm{H}), 2.66(\mathrm{dd}, 1 \mathrm{H}, J=13.6,10.4 \mathrm{~Hz}) .{ }^{13} \mathrm{C}\left\{{ }^{1} \mathrm{H}\right\}$ NMR $\left(150 \mathrm{MHz}, \mathrm{CD}_{3} \mathrm{OD}\right) \delta 193.9,160.4,159.6,157.4,156.9$, 131.4, 131.0, 130.2, 116.2, 106.1, 90.5, 70.1, 61.2, 56.5, 56.2, 49.7, 33.1. HRMS (ESI) $m / z$ : $[\mathrm{M}+\mathrm{H}]^{+}$calcd for $\mathrm{C}_{19} \mathrm{H}_{20} \mathrm{O}_{6}$ 345.1338; found, 345.1332 .

3-(4'-Benzyloxy)-5,7,8-trimethoxychroman-4-one (20a). An ethyl acetate $(3 \mathrm{~mL})$ and $\mathrm{CH}_{2} \mathrm{Cl}_{2}(1 \mathrm{~mL})$ solution of (E)-3-(4-(benzyloxy)-benzylidene)-5,7,8-trimethoxychroman4-one (19a) (27 mg, $0.06 \mathrm{mmol})$ as well as $5 \%$ wet $\mathrm{Pd} / \mathrm{C}(3.5$ $\mathrm{mg}, 0.03 \mathrm{mmol}$ ) was placed under an atmosphere of hydrogen. After stirring for $1 \mathrm{~h}$, the mixture was filtered through a Celite pad. After the filtrate was concentrated under reduced pressure, purification of the residue via flash column chromatography on silica gel (ethyl acetate/ $n$-hexane/methanol $=1: 3: 0.1)$ afforded 3-(4'-(benzyloxy)benzyl)-5,7,8trimethoxychroman-4-one (20a) (25 mg, 93\%). ${ }^{1} \mathrm{H}$ NMR $\left(600 \mathrm{MHz}, \mathrm{CDCl}_{3}\right) \delta 7.43(\mathrm{~d}, 2 \mathrm{H}, J=7.3 \mathrm{~Hz}), 7.38(\mathrm{t}, 2 \mathrm{H}, J=$ $7.5 \mathrm{~Hz}), 7.32(\mathrm{t}, 1 \mathrm{H}, J=7.3 \mathrm{~Hz}), 7.15(\mathrm{~d}, 2 \mathrm{H}, J=8.6 \mathrm{~Hz}), 6.92$ $(\mathrm{d}, 2 \mathrm{H}, J=8.6 \mathrm{~Hz}), 6.12(\mathrm{~s}, 1 \mathrm{H}), 5.04(\mathrm{~s}, 2 \mathrm{H}), 4.35(\mathrm{dd}, 1 \mathrm{H}, J$ $=11.3,4.1 \mathrm{~Hz}), 4.18(\mathrm{dd}, 1 \mathrm{H}, J=11.3,7.5 \mathrm{~Hz}), 3.94(\mathrm{~s}, 3 \mathrm{H})$, $3.92(\mathrm{~s}, 3 \mathrm{H}), 3.79(\mathrm{~s}, 3 \mathrm{H}), 3.19(\mathrm{dd}, 1 \mathrm{H}, J=14.0,4.4 \mathrm{~Hz}), 2.77$ (m, 1H), $2.64(\mathrm{dd}, 1 \mathrm{H}, J=14.0,10.6 \mathrm{~Hz}) .{ }^{13} \mathrm{C}\left\{{ }^{1} \mathrm{H}\right\} \mathrm{NMR}$ $\left(150 \mathrm{MHz}, \mathrm{CDCl}_{3}\right) \delta 191.7,158.6,158.3,157.6,156.4,137.2$, $130.9,130.7,130.3,128.7,128.0,127.6,115.1,105.8,89.3$, 70.1, 69.3, 61.3, 56.3, 56.1, 48.7, 32.0. HRMS (ESI) $m / z:[\mathrm{M}+$ $\mathrm{H}]^{+}$calcd for $\mathrm{C}_{26} \mathrm{H}_{26} \mathrm{O}_{6}$ 435.1810; found, 435.1803 .

3-(3', 4' -Bis(benzyloxy)benzyl)-5,7,8-trimethoxychroman-4-one (20b). An ethyl acetate $(9 \mathrm{~mL})$ and $\mathrm{CH}_{2} \mathrm{Cl}_{2}$ (3 $\mathrm{mL})$ solution of (E)-3-(3',4'-bis(benzyloxy)-benzylidene)5,7,8-trimethoxychroman-4-one (19b) (82 mg, $0.15 \mathrm{mmol})$ as well as $5 \%$ wet $\mathrm{Pd} / \mathrm{C}(8.0 \mathrm{mg}, 0.07 \mathrm{mmol})$ was placed under an atmosphere of hydrogen. After stirring for $1 \mathrm{~h}$, the mixture was filtered through a Celite pad. After the filtrate was concentrated under reduced pressure, purification of the residue via flash column chromatography on silica gel (ethyl acetate $/ n$-hexane $/$ methanol $=1: 3: 0.1)$ afforded 3 - $\left(3^{\prime}, 4^{\prime}\right.$-bis(benzyloxy)benzyl)-5,7,8-trimethoxychroman-4-one (20b) (75 $\mathrm{mg}, 91 \%) .{ }^{1} \mathrm{H}$ NMR $\left(600 \mathrm{MHz}, \mathrm{CDCl}_{3}\right) \delta 7.47-7.43(\mathrm{~m}, 4 \mathrm{H})$, $7.38-7.34(\mathrm{~m}, 4 \mathrm{H}), 7.33-7.28(\mathrm{~m}, 2 \mathrm{H}), 6.87(\mathrm{~d}, 1 \mathrm{H}, J=8.1$ $\mathrm{Hz}), 6.82(\mathrm{~d}, 1 \mathrm{H}, J=2.0 \mathrm{~Hz}), 6.74(\mathrm{dd}, 1 \mathrm{H}, J=8.1,2.0 \mathrm{~Hz})$, $6.12(\mathrm{~s}, 1 \mathrm{H}), 5.15(\mathrm{~d}, 4 \mathrm{H}, J=6.2 \mathrm{~Hz}), 4.28(\mathrm{dd}, 1 \mathrm{H}, J=11.3$, $4.2 \mathrm{~Hz}$ ), 4.09 (dd, $1 \mathrm{H}, J=11.3,7.5 \mathrm{~Hz}), 3.94(\mathrm{~s}, 3 \mathrm{H}), 3.92(\mathrm{~s}$, $3 \mathrm{H}), 3.80(\mathrm{~s}, 3 \mathrm{H}), 3.15(\mathrm{dd}, 1 \mathrm{H}, J=14.0,4.3 \mathrm{~Hz}), 2.72(\mathrm{~m}$, $1 \mathrm{H}), 2.59(\mathrm{dd}, 1 \mathrm{H}, J=14.0,10.6 \mathrm{~Hz}) .{ }^{13} \mathrm{C}\left\{{ }^{1} \mathrm{H}\right\} \operatorname{NMR}(150$ $\left.\mathrm{MHz}, \mathrm{CDCl}_{3}\right) \delta 191.7,158.6,158.3,156.5,149.0,147.8,137.5$, $137.34,132.0,130.7,128.6,127.9,127.9,127.6,127.4,122.3$, $116.3,115.5,105.9,89.4,71.6,71.5,69.3,61.3,56.3,56.2,48.6$, 32.4. HRMS (ESI) $m / z:[\mathrm{M}+\mathrm{H}]^{+}$calcd for $\mathrm{C}_{33} \mathrm{H}_{32} \mathrm{O}_{7}$ 541.2226; found, 541.2224.

3-(3'-(Benzyloxy)-4'-methoxybenzyl)-5,7,8-trimethoxychroman-4-one (20c). An ethyl acetate $(9 \mathrm{~mL})$ and $\mathrm{CH}_{2} \mathrm{Cl}_{2}$ (3 mL) solution of (E)-3-(3-(benzyloxy)-4-methoxybenzylidene)-5,7,8-trimethoxychroman-4-one (19c) (0.16 g, $0.35 \mathrm{mmol})$ as well as $5 \%$ wet $\mathrm{Pd} / \mathrm{C}(18 \mathrm{mg}, 0.17 \mathrm{mmol})$ was placed under an atmosphere of hydrogen. After stirring for $1 \mathrm{~h}$, the mixture was filtered through a Celite pad. After the filtrate was concentrated under reduced pressure, purification of the residue via flash column chromatography on silica gel (ethyl acetate $/ n$-hexane $/$ methanol $=1: 3: 0.1)$ afforded 3-(3'-(benzyloxy)-4'-methoxybenzyl)-5,7,8-trimethoxychroman-4-one (20c) $(0.15 \mathrm{~g}, 94 \%) .{ }^{1} \mathrm{H} \mathrm{NMR}\left(600 \mathrm{MHz}, \mathrm{CDCl}_{3}\right) \delta 7.44(\mathrm{~d}$, $2 \mathrm{H}, J=7.6 \mathrm{~Hz}), 7.35(\mathrm{t}, 2 \mathrm{H}, J=7.6 \mathrm{~Hz}), 7.28(\mathrm{t}, 1 \mathrm{H}, J=7.4$ $\mathrm{Hz}), 6.83(\mathrm{~d}, 1 \mathrm{H}, J=8.1 \mathrm{~Hz}), 6.79-6.76(\mathrm{~m}, 2 \mathrm{H}), 6.12(\mathrm{~s}$, $1 \mathrm{H}), 5.14(\mathrm{~s}, 2 \mathrm{H}), 4.26(\mathrm{dd}, 1 \mathrm{H}, J=11.3,4.1 \mathrm{~Hz}), 4.07(\mathrm{dd}$ $1 \mathrm{H}, J=11.3,7.5 \mathrm{~Hz}), 3.94(\mathrm{~s}, 3 \mathrm{H}), 3.92(\mathrm{~s}, 3 \mathrm{H}), 3.87(\mathrm{~s}, 3 \mathrm{H})$, 3.79 (s, 3H), 3.14 (dd, 1H, J = 13.9, $4.2 \mathrm{~Hz}), 2.70$ (ddd, $1 \mathrm{H}, J$ $=11.6,8.3,4.2 \mathrm{~Hz}), 2.59(\mathrm{dd}, 1 \mathrm{H}, J=13.9,10.6 \mathrm{~Hz}) .{ }^{13} \mathrm{C}\left\{{ }^{1} \mathrm{H}\right\}$ NMR $\left(150 \mathrm{MHz}, \mathrm{CDCl}_{3}\right) \delta 191.7,158.6,158.3,156.5,148.6$, 148.2 , 137.2, 131.1, 130.6, 128.7, 128.0, 127.6, 122.1, 115.4, 112.1, 105.9, 89.4, 71.2, 69.3, 61.3, 56.3, 56.2, 56.2, 48.7, 32.4. HRMS (ESI) $m / z:[\mathrm{M}+\mathrm{H}]^{+}$calcd for $\mathrm{C}_{27} \mathrm{H}_{28} \mathrm{O}_{7}$ 465.1913; found, 465.1909 .

3-(4'-(Benzyloxy)benzyl)-5,7,8-trimethoxychroman4-ol ((cis)-21a). To a solution of 3-(4'-(benzyloxy)benzyl)5,7,8-trimethoxychroman-4-one (20a) $(30 \mathrm{mg}, 0.069 \mathrm{mmol})$ in anhydrous tetrahydrofuran (THF) $(2 \mathrm{~mL})$, L-selectride $(0.10$ $\mathrm{mL}, 1.0 \mathrm{M}$ in THF) was added dropwise at $-78^{\circ} \mathrm{C}$. After $1 \mathrm{~h}$, the reaction was completed and quenched with $\mathrm{NH}_{4} \mathrm{Cl}$, extracted with ethyl ether, and dried over anhydrous $\mathrm{Na}_{2} \mathrm{SO}_{4}$. The residue was purified by flash chromatography on silica gel (ethyl acetate $/ n$-hexane $=1: 1)$ to afford 3-(4'-(benzyloxy)benzyl)-5,7,8-trimethoxychroman-4-ol (cis-21a) (30 mg, 98\%).

(3R,4R)-3-(4'-(Benzyloxy)benzyl)-5,7,8-trimethoxychroman-4-ol ((3R,4R)-21a). DBU and formic acid (3:1 (v/ v)) were dissolved in acetonitrile. The solution was sparged with nitrogen for $15 \mathrm{~min}$. Separately, 3-(4'-(benzyloxy)benzyl)-5,7,8-trimethoxychroman-4-one (20a) (53 mg, 0.12 $\mathrm{mmol})$ and $\mathrm{RuCl}(p$-cymene $)[(R, R)-\mathrm{Ts}-\mathrm{DPEN}](22)(23 \mathrm{mg}$, $0.036 \mathrm{mmol})$ were dissolved in acetonitrile and then added to the mixture of DBU and formic acid. The mixture was stirred for $24 \mathrm{~h}$ at $50{ }^{\circ} \mathrm{C}$ and then quenched by adding saturated $\mathrm{NH}_{4} \mathrm{Cl}$ solution at ambient temperature. After extraction with diethyl ether, the organic layer was washed with an additional 
portion of saturated $\mathrm{NaHCO}_{3}$ solution, dried over anhydrous $\mathrm{Na}_{2} \mathrm{SO}_{4}$, and concentrated under reduced pressure. The residue was purified by flash column chromatography on silica gel (ethyl acetate $/ n$-hexane/methanol $=1: 1: 0.1$ ) to afford (3R,4R)-3-(4'-(benzyloxy)benzyl)-5,7,8-trimethoxychroman4-ol $((3 R, 4 R)-21 \mathrm{a})(50 \mathrm{mg}, 94 \%)[\alpha]_{\mathrm{D}}^{24}=+100$ (c 0.10 , $\left.\mathrm{CH}_{3} \mathrm{OH}\right)$. ee $\%=92 \%$ ee. ${ }^{1} \mathrm{H} \mathrm{NMR}\left(600 \mathrm{MHz}, \mathrm{CDCl}_{3}\right) \delta 7.44$ $(\mathrm{d}, 2 \mathrm{H}, J=7.3 \mathrm{~Hz}), 7.39(\mathrm{t}, 2 \mathrm{H}, J=7.5 \mathrm{~Hz}), 7.33(\mathrm{t}, 1 \mathrm{H}, J=$ $7.3 \mathrm{~Hz}), 7.20(\mathrm{~d}, 2 \mathrm{H}, J=8.6 \mathrm{~Hz}), 6.94(\mathrm{~d}, 2 \mathrm{H}, J=8.6 \mathrm{~Hz})$, $6.09(\mathrm{~s}, 1 \mathrm{H}), 5.06(\mathrm{~s}, 2 \mathrm{H}), 4.73(\mathrm{~d}, 1 \mathrm{H}, J=2.5 \mathrm{~Hz}), 4.19-4.13$ (m, 1H), 4.07-4.01 (m, 1H), $3.87(\mathrm{~s}, 3 \mathrm{H}), 3.81(\mathrm{~s}, 3 \mathrm{H}), 3.77$ (s, 3H), $2.90(\mathrm{dd}, 1 \mathrm{H}, J=13.8,8.4 \mathrm{~Hz}), 2.63(\mathrm{dd}, 1 \mathrm{H}, J=13.8$, $7.3 \mathrm{~Hz}), 2.17(\mathrm{~m}, 1 \mathrm{H}) .{ }^{13} \mathrm{C}\left\{{ }^{1} \mathrm{H}\right\} \operatorname{NMR}\left(150 \mathrm{MHz}, \mathrm{CDCl}_{3}\right) \delta$ $157.4,154.2,153.3,149.0,137.3,131.9,131.5,130.2,128.7$, $128.1,127.6,115.0,107.9,88.6,70.2,65.5,61.2,59.9,56.4$, 55.67, 40.1, 32.0. HRMS (ESI) $\mathrm{m} / z:[\mathrm{M}+\mathrm{H}]^{+}$calcd for $\mathrm{C}_{26} \mathrm{H}_{28} \mathrm{O}_{6}$ 541.2226; found, 541.2224. $T_{\mathrm{R}}=10 \mathrm{~min}$ (chiral IC, $n$-hexane $/ \mathrm{EtOH}=60: 40)$.

3-(3', 4' -Bis(benzyloxy)benzyl)-5,7,8-trimethoxychroman-4-ol ((cis)-21b). To a solution of 3-( $3^{\prime}, 4^{\prime}$-bis(benzyloxy)benzyl)-5,7,8-trimethoxychroman-4-one (20b) (30 $\mathrm{mg}, 0.056 \mathrm{mmol})$ in anhydrous THF $(2 \mathrm{~mL})$, L-selectride (0.08 $\mathrm{mL}, 1.0 \mathrm{M}$ in THF) was added dropwise at $-78{ }^{\circ} \mathrm{C}$. After $1 \mathrm{~h}$, the reaction was completed, quenched with saturated $\mathrm{NH}_{4} \mathrm{Cl}$ solution, extracted with ethyl ether, and dried over anhydrous $\mathrm{Na}_{2} \mathrm{SO}_{4}$. The residue was purified by flash chromatography on silica gel (ethyl acetate $/ n$-hexane $=1: 1$ ) to afford 3-( $3^{\prime}, 4^{\prime}$-bis(benzyloxy)benzyl)-5,7,8-trimethoxychroman-4-ol (cis-21b) (26 mg, 85\%).

(3R,4R)-3-(3', $4^{\prime}$-Bis(benzyloxy)benzyl)-5,7,8-trimethoxychroman-4-ol $((3 R, 4 R)-21 \mathrm{~b})$. DBU and formic acid $(3: 1(\mathrm{v} / \mathrm{v}))$ were dissolved in acetonitrile. The solution was sparged with nitrogen for $15 \mathrm{~min}$. Separately, 3- $\left(3^{\prime}, 4^{\prime}\right.$ bis(benzyloxy)benzyl)-5,7,8-trimethoxychroman-4-one (20b) (30 $\mathrm{mg}, 0.06 \mathrm{mmol})$ and $\mathrm{RuCl}(p$-cymene $)[(R, R)-\mathrm{Ts}-\mathrm{DPEN}]$ (22) $(11 \mathrm{mg}, 0.02 \mathrm{mmol})$ were dissolved in acetonitrile and then added to the mixture of DBU and formic acid. The mixture was stirred for $24 \mathrm{~h}$ at $50{ }^{\circ} \mathrm{C}$ and then quenched by adding saturated $\mathrm{NH}_{4} \mathrm{Cl}$ solution at ambient temperature. After extraction with diethyl ether, the organic layer was washed with an additional portion of saturated $\mathrm{NaHCO}_{3}$ solution, dried over anhydrous $\mathrm{Na}_{2} \mathrm{SO}_{4}$, and concentrated under reduced pressure. The residue was purified by flash column chromatography on silica gel (ethyl acetate/ $n$-hexane/ methanol = 1:1:0.1) to afford $(3 R, 4 R)-3-\left(3^{\prime}, 4^{\prime}\right.$-bis(benzyloxy)benzyl)-5,7,8-trimethoxychroman-4-ol ((3R,4R)-21b) $(28 \mathrm{mg}$, 94\%) $[\alpha]_{\mathrm{D}}^{24}=+103\left(c 0.096, \mathrm{CH}_{3} \mathrm{OH}\right)$. ee $\%=95 \%$ ee. ${ }^{1} \mathrm{H}$ NMR $\left(600 \mathrm{MHz}, \mathrm{CDCl}_{3}\right) \delta 7.45(\mathrm{dd}, 4 \mathrm{H}, J=7.2,1.9 \mathrm{~Hz})$, $7.36(\mathrm{dt}, 4 \mathrm{H}, J=15.2,7.6 \mathrm{~Hz}), 7.30(\mathrm{dd}, 2 \mathrm{H}, J=14.6,7.3 \mathrm{~Hz})$, $6.89(\mathrm{~d}, 1 \mathrm{H}, J=8.2 \mathrm{~Hz}), 6.87(\mathrm{~d}, 1 \mathrm{H}, J=2.0 \mathrm{~Hz}), 6.78(\mathrm{dd}$, $1 \mathrm{H}, J=8.2,2.0 \mathrm{~Hz}$ ), 6.09 (s, 1H), $5.14(\mathrm{dd}, 4 \mathrm{H}, J=10.7,2.7$ $\mathrm{Hz}), 4.65$ (s, 1H), 4.10 (ddd, $1 \mathrm{H}, J=10.4,3.5,1.0 \mathrm{~Hz}), 4.03-$ $3.98(\mathrm{~m}, 1 \mathrm{H}), 3.87(\mathrm{~s}, 3 \mathrm{H}), 3.81(\mathrm{~s}, 3 \mathrm{H}), 3.78(\mathrm{~s}, 3 \mathrm{H}), 2.83$ $(\mathrm{dd}, 1 \mathrm{H}, J=13.8,8.5 \mathrm{~Hz}), 2.59(\mathrm{dd}, 1 \mathrm{H}, J=13.8,7.2 \mathrm{~Hz})$, 2.16-2.09 (m, 1H). ${ }^{13} \mathrm{C}\left\{{ }^{1} \mathrm{H}\right\}$ NMR $\left(150 \mathrm{MHz}, \mathrm{CDCl}_{3}\right) \delta$ $154.2,153.3,149.0,147.6,137.6,137.5,133.0,131.5,128.6$, $128.6,127.9,127.6,127.5,122.2,116.6,115.5,107.9,88.6$, 71.6, 71.6, 65.5, 61.2, 59.8, 56.4, 55.7, 40.0, 32.4. HRMS (ESI) $m / z:[\mathrm{M}+\mathrm{Na}]^{+}$calcd for $\mathrm{C}_{33} \mathrm{H}_{34} \mathrm{O}_{7}$ 565.2202; found, 565.2204. $T_{\mathrm{R}}=30 \mathrm{~min}$ (chiral ID, $n$-hexane $/ \mathrm{IPA} / \mathrm{EtOH}=$ 60:20:20).

3-(3'-(Benzyloxy)-4'-methoxybenzyl)-5,7,8-trimethoxychroman-4-ol ((cis)-21c). To a solution of 3-( $3^{\prime}-$ (benzyloxy)-4' -methoxybenzyl)-5,7,8-trimethoxychroman-4one $(20 \mathrm{c})(30 \mathrm{mg}, 0.065 \mathrm{mmol})$ in anhydrous THF $(2 \mathrm{~mL})$, Lselectride $(0.10 \mathrm{~mL}, 1.0 \mathrm{M}$ in $\mathrm{THF})$ was added dropwise at $-78{ }^{\circ} \mathrm{C}$. After $1 \mathrm{~h}$, the reaction was completed and quenched with $\mathrm{NH}_{4} \mathrm{Cl}$, extracted with ethyl ether, and dried over anhydrous $\mathrm{Na}_{2} \mathrm{SO}_{4}$. The residue was purified by flash chromatography on silica gel (ethyl acetate $/ n$-hexane $=1: 1$ ) to afford 3-(3'-(benzyloxy)-4'-methoxybenzyl)-5,7,8-trimethoxychroman-4-ol (cis-21c) (30 mg, 99\%).

(3R,4R)-3-(3'-(Benzyloxy)-4' -methoxybenzyl)-5,7,8trimethoxychroman-4-ol $((3 R, 4 R)-21 \mathrm{c})$. DBU and formic acid $(3: 1(\mathrm{v} / \mathrm{v}))$ were dissolved in acetonitrile. The solution was sparged with nitrogen for $15 \mathrm{~min}$. Separately, 3-(3'(benzyloxy)-4'-methoxybenzyl)-5,7,8-trimethoxychroman-4one (20c) (86 mg, $0.19 \mathrm{mmol})$ and $\operatorname{RuCl}(p$-cymene $)[(R, R)$ Ts-DPEN] (22) (35 mg, $0.06 \mathrm{mmol})$ were dissolved in acetonitrile and then added to the mixture of DBU and formic acid. The mixture was stirred for $24 \mathrm{~h}$ at $50{ }^{\circ} \mathrm{C}$ and then quenched by adding saturated $\mathrm{NH}_{4} \mathrm{Cl}$ solution at ambient temperature. After extraction with diethyl ether, the organic layer was washed with an additional portion of saturated $\mathrm{NaHCO}_{3}$ solution, dried over $\mathrm{Na}_{2} \mathrm{SO}_{4}$, and concentrated under reduced pressure. The residue was purified by flash column chromatography on silica gel (ethyl acetate $/ n$-hexane/methanol $=1: 1: 0.1)$ to afford $(3 R, 4 R)-3-\left(3^{\prime}\right.$-(benzyloxy)- $4^{\prime}$ methoxybenzyl)-5,7,8-trimethoxy-chroman-4-ol ((3R,4R)21c) $(84 \mathrm{mg}, 97 \%)[\alpha]_{\mathrm{D}}^{24}=+92\left(c 0.098, \mathrm{CH}_{3} \mathrm{OH}\right)$. ee $\%=$ $99 \%$ ee. ${ }^{1} \mathrm{H}$ NMR $\left(600 \mathrm{MHz}, \mathrm{CDCl}_{3}\right) \delta 7.44(\mathrm{~d}, 2 \mathrm{H}, J=7.3$ $\mathrm{Hz}), 7.35(\mathrm{t}, 2 \mathrm{H}, J=7.6 \mathrm{~Hz}), 7.29-7.25(\mathrm{~m}, 1 \mathrm{H}), 6.86-6.81$ $(\mathrm{m}, 3 \mathrm{H}), 6.08(\mathrm{~s}, 1 \mathrm{H}), 5.14(\mathrm{~s}, 2 \mathrm{H}), 4.63(\mathrm{~d}, 1 \mathrm{H}, J=2.8 \mathrm{~Hz})$, 4.11-4.07 (m, 1H), 4.02-3.97 (m, 1H), $3.88(\mathrm{~s}, 3 \mathrm{H}), 3.86(\mathrm{~s}$, $3 \mathrm{H}), 3.81(\mathrm{~s}, 3 \mathrm{H}), 3.78(\mathrm{~s}, 3 \mathrm{H}), 2.81(\mathrm{dd}, 1 \mathrm{H}, J=13.8,8.6$ $\mathrm{Hz}), 2.58(\mathrm{dd}, 1 \mathrm{H}, J=13.8,7.2 \mathrm{~Hz}), 2.15-2.08(\mathrm{~m}, 1 \mathrm{H})$. ${ }^{13} \mathrm{C}\left\{{ }^{1} \mathrm{H}\right\}$ NMR $\left(150 \mathrm{MHz}, \mathrm{CDCl}_{3}\right) \delta 154.2,153.3,148.91$, $148.3,148.1,137.4,132.0,131.5,128.6,127.9,127.5,121.9$, 115.6, 112.1, 107.8, 88.5, 71.2, 65.5, 61.1, 59.8, 56.4, 56.2, 55.6, 40.0, 32.3. HRMS (ESI) $m / z:[\mathrm{M}+\mathrm{H}]^{+}$calcd for $\mathrm{C}_{27} \mathrm{H}_{30} \mathrm{O}_{7}$ 497.2070; found, 467.2063. $T_{\mathrm{R}}=26 \mathrm{~min}$ (chiral ID, $n$-hexane/ $\mathrm{IPA} / \mathrm{EtOH}=60: 20: 20)$.

(R)-3-(4'-(Benzyloxy)benzyl)-5,7,8-trimethoxychroman-4-one $((R)-20 a)$. A solution of $(3 R, 4 R)-3-\left(4^{\prime}-\right.$ (benzyloxy)benzyl)-5,7,8-trimethoxychroman-4-ol ((3R,4R)21a) $(0.11 \mathrm{~g}, 0.24 \mathrm{mmol})$ in $\mathrm{CH}_{2} \mathrm{Cl}_{2}(5 \mathrm{~mL})$ was treated with molecular sieves ( $4 \AA, 0.10 \mathrm{~g}$ ) and $N$-methylmorpholine $\mathrm{N}$-oxide $(0.1 \mathrm{~g}, 0.84 \mathrm{mmol})$, and the mixture was stirred for 15 $\mathrm{min}$. Then, tetrapropylammonium perruthenate $(85 \mathrm{mg}, 0.24$ $\mathrm{mmol}$ ) was added, and stirring was continued at ambient temperature for $30 \mathrm{~min}$. The reaction mixture was diluted with diethyl ether, washed with water, dried over anhydrous $\mathrm{Na}_{2} \mathrm{SO}_{4}$, and concentrated under reduced pressure. The residue was purified by flash column chromatography on silica gel (ethyl acetate $/ n$-hexane $=1: 3$ ) to afford $(R)-3-\left(4^{\prime}\right.$ (benzyloxy)benzyl)-5,7,8-trimethoxychroman-4-one $((R)$ 20a) $(0.10 \mathrm{~g}, 97 \%)[\alpha]_{\mathrm{D}}^{24}=-83\left(c 0.072, \mathrm{CH}_{3} \mathrm{OH}\right)$. ee $\%=$ $92 \%$ ee. ${ }^{1} \mathrm{H}$ NMR $\left(600 \mathrm{MHz}, \mathrm{CDCl}_{3}\right) \delta 7.43(\mathrm{~d}, 2 \mathrm{H}, J=7.3$ $\mathrm{Hz}), 7.38(\mathrm{t}, 2 \mathrm{H}, J=7.5 \mathrm{~Hz}), 7.32(\mathrm{t}, 1 \mathrm{H}, J=7.3 \mathrm{~Hz}), 7.15(\mathrm{~d}$, $2 \mathrm{H}, J=8.6 \mathrm{~Hz}), 6.92(\mathrm{~d}, 2 \mathrm{H}, J=8.6 \mathrm{~Hz}), 6.12(\mathrm{~s}, 1 \mathrm{H}), 5.05(\mathrm{~s}$, $2 \mathrm{H}), 4.35(\mathrm{dd}, 1 \mathrm{H}, J=11.3,4.1 \mathrm{~Hz}), 4.18(\mathrm{dd}, 1 \mathrm{H}, J=11.3$, $7.5 \mathrm{~Hz}$ ), 3.94 (s, $3 \mathrm{H}), 3.92$ (s, $3 \mathrm{H}), 3.79$ (s, $3 \mathrm{H}), 3.19$ (dd, $1 \mathrm{H}$, $J=14.0,4.3 \mathrm{~Hz}), 2.77(\mathrm{~m}, 1 \mathrm{H}), 2.64(\mathrm{dd}, 1 \mathrm{H}, J=14.0,10.6$ $\mathrm{Hz}) .{ }^{13} \mathrm{C}\left\{{ }^{1} \mathrm{H}\right\}$ NMR $\left(150 \mathrm{MHz}, \mathrm{CDCl}_{3}\right) \delta 191.7,158.6,158.3$, $157.6,156.4,137.2,130.9,130.7,130.3,128.7,128.1,127.6$, 115.1, 105.8, 89.4, 70.2, 69.3, 61.3, 56.3, 56.2, 48.7, 32.0. 
HRMS (ESI) $m / z$ : $[\mathrm{M}+\mathrm{H}]^{+}$calcd for $\mathrm{C}_{26} \mathrm{H}_{26} \mathrm{O}_{6} 435.1808$; found, 435.1803. $T_{\mathrm{R}}=21 \mathrm{~min}$ (chiral ID, $n$-hexane $/ \mathrm{EtOH}=$ $70: 30)$.

(R)-3-(3',4'-Bis(benzyloxy)benzyl)-5,7,8-trimethoxychroman-4-one $((R)-20 \mathrm{~b})$. A solution of $(3 R, 4 R)-3-\left(3^{\prime}, 4^{\prime}\right.$ bis(benzyloxy)benzyl)-5,7,8-trimethoxychroman-4-ol $((3 R, 4 R)-21 \mathbf{b})(17 \mathrm{mg}, 0.03 \mathrm{mmol})$ in $\mathrm{CH}_{2} \mathrm{Cl}_{2}(2 \mathrm{~mL})$ was treated with molecular sieves (4 $\AA, 13 \mathrm{mg})$ and $\mathrm{N}$ methylmorpholine $\mathrm{N}$-oxide $(13 \mathrm{mg}, 0.11 \mathrm{mmol})$, and the mixture was stirred for $15 \mathrm{~min}$. Then, tetrapropylammonium perruthenate (11 mg, $0.03 \mathrm{mmol}$ ) was added, and stirring was continued at ambient temperature for $30 \mathrm{~min}$. The reaction mixture was diluted with diethyl ether, washed with water, dried over anhydrous $\mathrm{Na}_{2} \mathrm{SO}_{4}$, and concentrated under reduced pressure. The residue was purified by flash column chromatography on silica gel (ethyl acetate $/ n$-hexane $=1: 3$ ) to afford (R)-3-(3',4'-bis(benzyloxy)benzyl)-5,7,8-trimethoxychroman-4-one $((R)-20 \mathrm{~b})(69 \mathrm{mg}, 93 \%)[\alpha]_{\mathrm{D}}^{24}=-87(c$ $\left.0.058, \mathrm{CH}_{3} \mathrm{OH}\right)$. ee $\%=87 \%$ ee. ${ }^{1} \mathrm{H}$ NMR $\left(600 \mathrm{MHz} \mathrm{CDCl}_{3}\right)$ $\delta 7.44(\mathrm{dd}, 4 \mathrm{H}, J=7.1,4.1 \mathrm{~Hz}), 7.35(\mathrm{td}, 4 \mathrm{H}, J=7.5,2.5 \mathrm{~Hz})$, $7.30(\mathrm{td}, 2 \mathrm{H}, J=7.2,5.5 \mathrm{~Hz}), 6.86(\mathrm{~d}, 1 \mathrm{H}, J=8.2 \mathrm{~Hz}), 6.82(\mathrm{~d}$, $1 \mathrm{H}, J=1.9 \mathrm{~Hz}), 6.74(\mathrm{dd}, 1 \mathrm{H}, J=8.2,1.9 \mathrm{~Hz}), 6.12(\mathrm{~s}, 1 \mathrm{H})$, $5.15(\mathrm{~s}, 2 \mathrm{H}), 5.13(\mathrm{~s}, 2 \mathrm{H}), 4.27(\mathrm{dd}, 1 \mathrm{H}, J=11.3,4.1 \mathrm{~Hz}), 4.08$ $(\mathrm{dd}, 1 \mathrm{H}, J=11.3,7.5 \mathrm{~Hz}), 3.94(\mathrm{~s}, 3 \mathrm{H}), 3.92(\mathrm{~s}, 3 \mathrm{H}), 3.79$ (s, $3 \mathrm{H}), 3.14(\mathrm{dd}, 1 \mathrm{H}, J=14.0,4.3 \mathrm{~Hz}), 2.71(\mathrm{~m}, 1 \mathrm{H}), 2.59$ (dd, $1 \mathrm{H}, J=14.0,10.6 \mathrm{~Hz}) .{ }^{13} \mathrm{C}\left\{{ }^{1} \mathrm{H}\right\} \mathrm{NMR}\left(150 \mathrm{MHz}, \mathrm{CDCl}_{3}\right) \delta$ $191.7,158.6,158.3,156.4,149.0,147.8,137.5,137.3,132.0$, $130.7,128.6,127.9,127.9,127.6,127.4,122.3,116.3,115.5$, 105.9, 89.3, 71.6, 71.5, 69.3, 61.3, 56.3, 56.2, 48.6, 32.4. HRMS (ESI) $m / z:[\mathrm{M}+\mathrm{H}]^{+}$calcd for $\mathrm{C}_{33} \mathrm{H}_{32} \mathrm{O}_{7}$ 541.2226; found, 541.2222. $T_{\mathrm{R}}=27 \mathrm{~min}$ (chiral ID, $n$-hexane $/ \mathrm{EtOH}=70: 40$ ).

(R)-3-(3'-(Benzyloxy)-4'-methoxybenzyl)-5,7,8-trimethoxychroman-4-one $((R)-20 \mathrm{c})$. A solution of $(3 R, 4 R)-3$ (3'-(benzyloxy)-4' -methoxybenzyl)-5,7,8-trimethoxy-chroman-4-ol $((3 R, 4 R)-21 \mathrm{c})(70 \mathrm{mg}, 0.15 \mathrm{mmol})$ in $\mathrm{CH}_{2} \mathrm{Cl}_{2}$ (3 $\mathrm{mL})$ was treated with molecular sieves $(4 \AA, 60 \mathrm{mg})$ and $N$ methylmorpholine $\mathrm{N}$-oxide $(60 \mathrm{mg}, 0.51 \mathrm{mmol})$, and the mixture was stirred for $15 \mathrm{~min}$. Then, tetrapropylammonium perruthenate $(54 \mathrm{mg}, 0.15 \mathrm{mmol}$ ) was added, and stirring was continued at ambient temperature for $20 \mathrm{~min}$. The reaction mixture was diluted with diethyl ether, washed with water, dried over anhydrous $\mathrm{Na}_{2} \mathrm{SO}_{4}$, and concentrated under reduced pressure. The residue was purified by flash column chromatography on silica gel (ethyl acetate $/ n$-hexane $=1: 3$ ) to afford (R)-3-(3'-(benzyloxy)-4'-methoxybenzyl)-5,7,8-trimethoxychroman-4-one $((R)-20 \mathrm{c})(69 \mathrm{mg}, 99 \%)[\alpha]_{\mathrm{D}}^{24}=-57$ (c $\left.0.088, \mathrm{CH}_{3} \mathrm{OH}\right)$. ee $\%=84 \%$ ee. ${ }^{1} \mathrm{H} \mathrm{NMR}(600 \mathrm{MHz}$, $\left.\mathrm{CDCl}_{3}\right) \delta 7.43(\mathrm{~d}, 2 \mathrm{H}, J=7.5 \mathrm{~Hz}), 7.34(\mathrm{t}, 2 \mathrm{H}, J=7.5 \mathrm{~Hz})$, $7.29-7.25(\mathrm{~m}, 1 \mathrm{H}), 6.82(\mathrm{~d}, 1 \mathrm{H}, J=7.9 \mathrm{~Hz}), 6.77(\mathrm{~m}, 2 \mathrm{H})$, $6.11(\mathrm{~s}, 1 \mathrm{H}), 5.12(\mathrm{~s}, 2 \mathrm{H}), 4.25(\mathrm{dd}, 1 \mathrm{H}, J=11.2,3.7 \mathrm{~Hz}), 4.06$ $(\mathrm{dd}, 1 \mathrm{H}, J=11.2,7.7 \mathrm{~Hz}), 3.93(\mathrm{~s}, 3 \mathrm{H}), 3.90(\mathrm{~s}, 3 \mathrm{H}), 3.85$ (s, $3 \mathrm{H}), 3.78(\mathrm{~s}, 3 \mathrm{H}), 3.76-3.76(\mathrm{~m}, 1 \mathrm{H}), 3.12(\mathrm{dd}, 1 \mathrm{H}, J=13.6$, $3.8 \mathrm{~Hz}), 2.69(\mathrm{~m}, 1 \mathrm{H}), 2.58(\mathrm{dd}, 1 \mathrm{H}, J=13.610 .9 \mathrm{~Hz})$. ${ }^{13} \mathrm{C}\left\{{ }^{1} \mathrm{H}\right\} \mathrm{NMR}\left(150 \mathrm{MHz}, \mathrm{CDCl}_{3}\right) \delta 191.6,158.6,158.3$, 156.4, 148.5, 148.1, 137.1, 131.0, 130.6, 128.6, 127.9, 12 127.5, $122.0,115.3,112.0,105.8,89.3,71.1,69.2,61.3,56.3,56.2$, 56.1, 48.6, 32.4. HRMS (ESI) $m / z:[\mathrm{M}+\mathrm{H}]^{+}$calcd for $\mathrm{C}_{27} \mathrm{H}_{28} \mathrm{O}_{7}$ 465.1913; found, 465.1908. $\mathrm{T}_{\mathrm{R}}=24 \mathrm{~min}$ (chiral ID, $n$-hexane/EtOH = 70:40).

$(R)$-7-Methyl-3,9-dihydropunctatin $((R)-4)$. To a solution of $(R)$-3-(4-(benzyloxy)benzyl)-5,7,8-trimethoxychroman-4-one $((R)-20 a)(32 \mathrm{mg}, 0.08 \mathrm{mmol})$ in $\mathrm{CH}_{2} \mathrm{Cl}_{2}$ (2 $\mathrm{mL})$ was added boron trichloride $(0.23 \mathrm{~mL}, 1.0 \mathrm{M}$ solution in
$\mathrm{CH}_{2} \mathrm{Cl}_{2}$ ) at $-78{ }^{\circ} \mathrm{C}$. After stirring for $40 \mathrm{~min}$, the reaction mixture was diluted with $\mathrm{CH}_{2} \mathrm{Cl}_{2}$, washed with water, dried over anhydrous $\mathrm{Na}_{2} \mathrm{SO}_{4}$, and concentrated under reduced pressure. The residue was purified by flash column chromatography on silica gel (ethyl acetate/n-hexane/methanol $=1: 1: 0.1)$ to afford $(R)$-7-methyl-3,9-dihydropunctation $((R)-4)$ (23 mg, 93\%). $[\alpha]_{\mathrm{D}}^{24}=-74\left(c 0.068, \mathrm{CH}_{3} \mathrm{OH}\right)$. ee $\%=$ $86 \%$ ee. ${ }^{1} \mathrm{H}$ NMR $\left(600 \mathrm{MHz}, \mathrm{CD}_{3} \mathrm{OD}\right) \delta 7.03(\mathrm{~d}, 2 \mathrm{H}, J=8.5$ $\mathrm{Hz}), 6.70(\mathrm{~d}, 2 \mathrm{H}, J=8.5 \mathrm{~Hz}), 6.13(\mathrm{~s}, 1 \mathrm{H}), 4.29(\mathrm{dd}, 1 \mathrm{H}, J=$ $11.4,4.3 \mathrm{~Hz}), 4.14(\mathrm{dd}, 1 \mathrm{H}, J=11.4,7.3 \mathrm{~Hz}), 3.84(\mathrm{~s}, 3 \mathrm{H})$, $3.66(\mathrm{~s}, 3 \mathrm{H}), 3.08(\mathrm{dd}, 1 \mathrm{H}, J=14.0,4.7 \mathrm{~Hz}), 2.83(\mathrm{~m}, 1 \mathrm{H})$, $2.64(\mathrm{dd}, 1 \mathrm{H}, J=14.0,10.1 \mathrm{~Hz}) .{ }^{13} \mathrm{C}\left\{{ }^{1} \mathrm{H}\right\} \mathrm{NMR}(150 \mathrm{MHz}$, $\left.\mathrm{CD}_{3} \mathrm{OD}\right) \delta 199.8,162.5,161.3,157.1,154.9,131.0,130.2$, 129.8, 116.2, 103.1, 93.6, 70.4, 61.3, 56.5, 48.0, 32.7. HRMS (ESI) $m / z:[\mathrm{M}+\mathrm{H}]^{+}$calcd for $\mathrm{C}_{18} \mathrm{H}_{18} \mathrm{O}_{6} 331.1181$; found, 331.1181. $T_{\mathrm{R}}=7.7 \mathrm{~min}$ (chiral IB, $0.1 \%$ diethanolamine (DEA) in $n$-hexane/EtOH $=70: 30)$.

(R)-7-O-Methyl-3'-hydroxy-3,9-dihydropunctatin $((R)-$ $5)$. To a solution of (R)-3-(3,4-bis(benzyloxy)benzyl)-5,7,8trimethoxychroman-4-one $((R)-20 \mathrm{~b})(30 \mathrm{mg}, 0.06 \mathrm{mmol})$ in $\mathrm{CH}_{2} \mathrm{Cl}_{2}(3 \mathrm{~mL})$ was added boron trichloride $(0.16 \mathrm{~mL}, 1.0 \mathrm{M}$ solution in $\mathrm{CH}_{2} \mathrm{Cl}_{2}$ ) at $-78{ }^{\circ} \mathrm{C}$. After stirring for $90 \mathrm{~min}$, the reaction mixture was diluted with $\mathrm{CH}_{2} \mathrm{Cl}_{2}$, washed with water, dried over anhydrous $\mathrm{Na}_{2} \mathrm{SO}_{4}$, and concentrated under reduced pressure. The residue was purified by flash column chromatography on silica gel (ethyl acetate/ $n$-hexane/methanol $=1: 1: 0.1)$ to afford (R)-7-O-methyl-3'-hydroxy-3,9dihydropunctatin $((R)-5)(17 \mathrm{mg}, 89 \%) \cdot[\alpha]_{\mathrm{D}}^{24}=-75(c$ 0.066, $\left.\mathrm{CH}_{3} \mathrm{OH}\right)$. ee $\%=95 \%$ ee. ${ }^{1} \mathrm{H} \mathrm{NMR}\left(600 \mathrm{MHz} \mathrm{CDCl}_{3}\right)$ $\delta 12.02(\mathrm{~s}, 1 \mathrm{H}), 6.78(\mathrm{~d}, 1 \mathrm{H}, J=8.0 \mathrm{~Hz}), 6.73(\mathrm{~d}, 1 \mathrm{H}, J=2.0$ $\mathrm{Hz}), 6.63(\mathrm{dd}, 1 \mathrm{H}, J=8.0,2.0 \mathrm{~Hz}), 6.08(\mathrm{~s}, 1 \mathrm{H}), 5.54(\mathrm{br} \mathrm{s}$, $1 \mathrm{H}), 5.43$ (br s, $1 \mathrm{H}), 4.32(\mathrm{dd}, 1 \mathrm{H}, J=11.4,4.2 \mathrm{~Hz}), 4.17$ (dd, $1 \mathrm{H}, J=11.5,6.9 \mathrm{~Hz}), 3.87(\mathrm{~s}, 3 \mathrm{H}), 3.76(\mathrm{~s}, 3 \mathrm{H}), 3.08(\mathrm{dd}, 1 \mathrm{H}$, $J=14.0,4.6 \mathrm{~Hz}), 2.79(\mathrm{~m}, 1 \mathrm{H}), 2.65(\mathrm{dd}, 1 \mathrm{H}, J=14.0,10.3$ $\mathrm{Hz}) .{ }^{13} \mathrm{C}\left\{{ }^{1} \mathrm{H}\right\} \mathrm{NMR}\left(150 \mathrm{MHz}, \mathrm{CDCl}_{3}\right) \delta 198.4,161.5,160.5$, $153.8,144.0,142.6,130.8,129.4,121.8,116.3,115.7,102.5$, 93.2, 69.5, 61.6, 56.4, 47.0, 32.4. HRMS (ESI) $m / z:[\mathrm{M}+\mathrm{H}]^{+}$ calcd for $\mathrm{C}_{18} \mathrm{H}_{18} \mathrm{O}_{7}$ 347.1131; found, 347.1125. $T_{\mathrm{R}}=26 \mathrm{~min}$ (chiral IG, $0.1 \%$ trifluoroacetic acid (TFA) in $n$-hexane/EtOH $=70: 30)$.

(R)-7,4'-Di-O-methyl-3' -hydroxy-3,9-dihydropunctatin $((R)-6)$. To a solution of $(R)-3-(3$-(benzyloxy)-4methoxybenzyl)-5,7,8-trimethoxy-chroman-4-one ( $(R)-20 \mathrm{c})$ (25 mg, $0.05 \mathrm{mmol})$ in $\mathrm{CH}_{2} \mathrm{Cl}_{2}(2 \mathrm{~mL})$ was added boron trichloride $\left(0.16 \mathrm{~mL}, 1.0 \mathrm{M}\right.$ solution in $\left.\mathrm{CH}_{2} \mathrm{Cl}_{2}\right)$ at $-78{ }^{\circ} \mathrm{C}$. After stirring for $1 \mathrm{~h}$, the reaction mixture was diluted with $\mathrm{CH}_{2} \mathrm{Cl}_{2}$, washed with water, dried over $\mathrm{Na}_{2} \mathrm{SO}_{4}$, and concentrated under reduced pressure. The residue was purified by flash column chromatography on silica gel (ethyl acetate $/ n$ hexane/methanol = 1:1:0.1) to afford $(R)-7,4^{\prime}$-di-O-methyl-3' hydroxy-3,9-dihydropunctatin $((R)-6)(18 \mathrm{mg}, 93 \%) .[\alpha]_{\mathrm{D}}^{24}=$ -48 (c $\left.0.10, \mathrm{CH}_{3} \mathrm{OH}\right)$. ee $\%=75 \%$ ee. ${ }^{1} \mathrm{H}$ NMR $(600 \mathrm{MHz}$, $\left.\mathrm{CDCl}_{3}\right) \delta 12.04(\mathrm{~s}, 1 \mathrm{H}), 6.80(\mathrm{~d}, 1 \mathrm{H}, J=2.1 \mathrm{~Hz}), 6.78(\mathrm{~d}, 1 \mathrm{H}$, $J=8.2 \mathrm{~Hz}), 6.69(\mathrm{dd}, 1 \mathrm{H}, J=8.2,2.1 \mathrm{~Hz}), 6.09(\mathrm{~s}, 1 \mathrm{H}), 5.62$ (s, $1 \mathrm{H}), 4.34(\mathrm{dd}, 1 \mathrm{H}, J=11.4,4.3 \mathrm{~Hz}), 4.19(\mathrm{dd}, 1 \mathrm{H}, J=11.4$, $7.3 \mathrm{~Hz}), 3.88(\mathrm{~s}, 3 \mathrm{H}), 3.87(\mathrm{~s}, 3 \mathrm{H}), 3.76(\mathrm{~s}, 3 \mathrm{H}), 3.15(\mathrm{dd}, 1 \mathrm{H}$, $J=14.0,4.6 \mathrm{~Hz}), 2.87-2.82(\mathrm{~m}, 1 \mathrm{H}), 2.67(\mathrm{dd}, 1 \mathrm{H}, J=14.0$, $10.4 \mathrm{~Hz}) .{ }^{13} \mathrm{C}\left\{{ }^{1} \mathrm{H}\right\}$ NMR $\left(150 \mathrm{MHz}, \mathrm{CDCl}_{3}\right) \delta 198.3,161.5$, $160.4,153.9,145.9,145.6,131.1,129.5,120.8,115.3,110.9$, 102.5, 93.2, 69.5, 61.5, 56.4, 56.2, 47.0, 32.3. HRMS (ESI) $\mathrm{m} /$ $z:[\mathrm{M}+\mathrm{H}]^{+}$calcd for $\mathrm{C}_{19} \mathrm{H}_{20} \mathrm{O}_{7}$ 361.1287; found, 361.1283. $T_{\mathrm{R}}=12 \mathrm{~min}$ (chiral IB, $0.1 \%$ diethanolamine (DEA) in $n$ hexane $/ \mathrm{EtOH}=70: 30)$. 
1-(4-(Benzyloxy)-2-hydroxy-3,6-dimethoxyphenyl)ethan-1-one (26). First step: To an acetone (20 mL) solution of 1-(4-(benzyloxy)-6-hydroxy-2,3-dimethoxyphenyl)ethan-1-one (23) (2.0 g, $6.6 \mathrm{mmol}), 2.0 \mathrm{M}$ in $t$-butyl methyl ether of solution iodomethane $(3.6 \mathrm{~mL}, 7.3 \mathrm{mmol})$ and $\mathrm{Cs}_{2} \mathrm{CO}_{3}(4.3 \mathrm{~g}, 13 \mathrm{mmol})$ were added. The reaction mixture was refluxed for $12 \mathrm{~h}$. After cooling to ambient temperature, the reaction mixture was diluted with ethyl acetate and the organic phase was washed with water and saturated $\mathrm{NH}_{4} \mathrm{Cl}$ solution, dried over anhydrous $\mathrm{Na}_{2} \mathrm{SO}_{4}$, and concentrated under reduced pressure. The residue was purified by flash column chromatography on silica gel (ethyl acetate/ $n$-hexane $=1: 3$ ) to afford 1-(4-(benzyloxy)-2,3,6-trimethoxyphenyl)ethan-1-one (2.0 g, 95\%). ${ }^{1} \mathrm{H}$ NMR $\left(600 \mathrm{MHz}^{\mathrm{CDCl}} \mathrm{CD}_{3}\right) \delta 7.44$ $(\mathrm{d}, J=7.2 \mathrm{~Hz}, 2 \mathrm{H}), 7.39(\mathrm{t}, J=7.3 \mathrm{~Hz}, 2 \mathrm{H}), 7.34(\mathrm{~d}, J=7.1$ $\mathrm{Hz}, 1 \mathrm{H}), 6.29$ (s, 1H), 5.14 (s, 2H), 3.90 (s, 3H), $3.83(\mathrm{~s}, 3 \mathrm{H})$, $3.71(\mathrm{~s}, 3 \mathrm{H}), 2.47(\mathrm{~s}, 3 \mathrm{H}) .{ }^{13} \mathrm{C}\left\{{ }^{1} \mathrm{H}\right\}$ NMR $\left(150 \mathrm{MHz} \mathrm{CDCl}_{3}\right)$ $\delta$ 201.4, 154.0, 152.5, 151.4, 136.9, 136.7, 128.8, 128.3, 127.4, $119.1,94.7,71.4,62.2,61.3,56.2,32.7$. HRMS (ESI) $\mathrm{m} / z$ : [M $+\mathrm{H}]^{+}$calcd for $\mathrm{C}_{18} \mathrm{H}_{20} \mathrm{O}_{5} 317.1389$; found, 317.1384. Second step: To a solution of 1-(4-(benzyloxy)-2,3,6trimethoxyphenyl)ethan-1-one $(1.1 \mathrm{~g}$, $5.4 \mathrm{mmol})$ in $\mathrm{CH}_{2} \mathrm{Cl}_{2}$ $(20 \mathrm{~mL})$ was added boron trichloride $(16 \mathrm{~mL}, 1.0 \mathrm{M}$ solution in $\mathrm{CH}_{2} \mathrm{Cl}_{2}$ ) at $-78{ }^{\circ} \mathrm{C}$. After stirring for $1 \mathrm{~h}$, the reaction mixture was diluted with $\mathrm{CH}_{2} \mathrm{Cl}_{2}$, washed with water, dried over anhydrous $\mathrm{Na}_{2} \mathrm{SO}_{4}$, and concentrated under reduced pressure. The residue was purified by flash column chromatography on silica gel (ethyl acetate $/ n$-hexane/methanol $=1: 5)$ to afford intermediates (24 and 25$)(1.0 \mathrm{~g}, 90 \%)$. Third step: To an acetone $(20 \mathrm{~mL})$ solution of intermediates (24 and 25) (1.0 g, $4.8 \mathrm{mmol})$, benzyl bromide ( $0.6 \mathrm{~mL}, 5.3$ $\mathrm{mmol})$ and $\mathrm{K}_{2} \mathrm{CO}_{3}(1.3 \mathrm{~g}, 9.6 \mathrm{mmol})$ were added. The reaction mixture was refluxed for $2 \mathrm{~h}$. After cooling to ambient temperature, the reaction mixture was diluted with ethyl acetate and the organic phase was washed with water and saturated $\mathrm{NH}_{4} \mathrm{Cl}$ solution, dried over anhydrous $\mathrm{Na}_{2} \mathrm{SO}_{4}$, and concentrated under reduced pressure. The residue was purified by flash column chromatography on silica gel (ethyl acetate $/ n$ hexane $=1: 2$ ) to afford 1-(4-(benzyloxy)-2-hydroxy-3,6dimethoxyphenyl)ethan-1-one (26) (1.0 g, 72\%). ${ }^{1} \mathrm{H}$ NMR $\left(600 \mathrm{MHz}, \mathrm{CDCl}_{3}\right) \delta 13.82(\mathrm{~s}, 1 \mathrm{H}), 7.43(\mathrm{~d}, J=7.4 \mathrm{~Hz}, 2 \mathrm{H})$, $7.39(\mathrm{t}, J=7.5 \mathrm{~Hz}, 2 \mathrm{H}), 7.33(\mathrm{t}, J=7.3 \mathrm{~Hz}, 1 \mathrm{H}), 5.97(\mathrm{~s}, 1 \mathrm{H})$, $5.22(\mathrm{~s}, 2 \mathrm{H}), 3.84(\mathrm{~s}, 3 \mathrm{H}), 3.78(\mathrm{~s}, 3 \mathrm{H}), 2.59(\mathrm{~s}, 3 \mathrm{H}) .{ }^{13} \mathrm{C}\left\{{ }^{1} \mathrm{H}\right\}$ NMR $\left(150 \mathrm{MHz}, \mathrm{CDCl}_{3}\right) \delta 203.9,159.2,158.8,157.6,136.4$, 131.2 , 128.8, 128.3, 127.3, 106.6, 88.6, 70.9, 60.9, 55.6, 33.3. HRMS (ESI) $m / z$ : $[\mathrm{M}+\mathrm{H}]^{+}$calcd for $\mathrm{C}_{17} \mathrm{H}_{18} \mathrm{O}_{5}$ 303.1232; found, 303.1227.

7-(Benzyloxy)-5,8-dimethoxy-4H-chromen-4-one (27). To a solution of 1-(4-(benzyloxy)-2-hydroxy-3,6dimethoxyphenyl)ethan-1-one (26) (1.0 g, $3.3 \mathrm{mmol})$ in DME $(20 \mathrm{~mL})$ was added $N, N$-dimethylformamide dimethyl acetal $(1.3 \mathrm{~mL}, 9.9 \mathrm{mmol})$. After stirring for $24 \mathrm{~h}$ at $80^{\circ} \mathrm{C}$, the mixture was cooled to $0{ }^{\circ} \mathrm{C}$ and $\mathrm{c}$ - $\mathrm{HCl}(5 \mathrm{~mL})$ was added. After stirring for $1 \mathrm{~h}$ at $50{ }^{\circ} \mathrm{C}$, the reaction mixture was diluted with ethyl acetate and the organic phase was washed with water and brine and dried over anhydrous $\mathrm{Na}_{2} \mathrm{SO}_{4}$. The solvent was removed under reduced pressure and purified by flash column chromatography on silica gel (ethyl acetate/ $\left.\mathrm{CH}_{2} \mathrm{Cl}_{2}=1: 3\right)$ to afford 7-(benzyloxy)-5,8-dimethoxy-4Hchromen-4-one (27) (0.97 g, 94\%). ${ }^{1} \mathrm{H}$ NMR (600 MHz, $\left.\mathrm{CDCl}_{3}\right) \delta 7.69(\mathrm{~d}, 1 \mathrm{H}, J=5.9 \mathrm{~Hz}), 7.45(\mathrm{~d}, 2 \mathrm{H}, J=7.4 \mathrm{~Hz})$, $7.40(\mathrm{t}, 2 \mathrm{H}, J=7.4 \mathrm{~Hz}), 7.35(\mathrm{t}, 1 \mathrm{H}, J=7.3 \mathrm{~Hz}), 6.45(\mathrm{~s}, 1 \mathrm{H})$, $6.17(\mathrm{~d}, 1 \mathrm{H}, J=5.9 \mathrm{~Hz}), 5.26(\mathrm{~s}, 2 \mathrm{H}), 3.90(\mathrm{~s}, 3 \mathrm{H}), 3.86(\mathrm{~s}$,
$3 \mathrm{H}) .{ }^{13} \mathrm{C}\left\{{ }^{1} \mathrm{H}\right\}$ NMR $\left(150 \mathrm{MHz}, \mathrm{CDCl}_{3}\right) \delta 176.9,156.3,155.6$, $152.9,152.5,136.1,131.2,128.9,128.6,127.4,114.2,110.5$, 94.7, 71.4, 61.8, 56.6. HRMS (ESI) $m / z:[\mathrm{M}+\mathrm{H}]^{+}$calcd for $\mathrm{C}_{18} \mathrm{H}_{16} \mathrm{O}_{5}$ 313.1076; found, 313.1071.

7-Hydroxy-5,8-dimethoxychroman-4-one (28). 7(Benzyloxy)-5,8-dimethoxy-4H-chromen-4-one (27) (0.30 g, $0.96 \mathrm{mmol})$ and $10 \% \mathrm{Pd} / \mathrm{C}(0.01 \mathrm{~g}, 0.10 \mathrm{mmol})$ in anhydrous methanol $(15 \mathrm{~mL})$ were placed under an atmosphere of hydrogen. After stirring for $4 \mathrm{~h}$, the mixture was filtered through a Celite pad. After the filtrate was concentrated under reduced pressure, purification of the residue via flash column chromatography on silica gel (ethyl acetate $/ \mathrm{CH}_{2} \mathrm{Cl}_{2}=1: 3$ ) afforded 7-hydroxy-5,8-dimethoxychroman-4-one (28) (0.22 g, 99\%). ${ }^{1} \mathrm{H}$ NMR (600 MHz, CD $\left.\mathrm{OD}\right) \delta 6.13(\mathrm{~s}, 1 \mathrm{H}), 4.48(\mathrm{t}$, $2 \mathrm{H}, J=6.4 \mathrm{~Hz}), 3.78(\mathrm{~s}, 3 \mathrm{H}), 3.74(\mathrm{~s}, 3 \mathrm{H}), 2.69(\mathrm{t}, 2 \mathrm{H}, J=6.4$ $\mathrm{Hz}) .{ }^{13} \mathrm{C}\left\{{ }^{1} \mathrm{H}\right\} \mathrm{NMR}\left(150 \mathrm{MHz}, \mathrm{CD}_{3} \mathrm{OD}\right) \delta 191.9,159.6$, 159.3 , 158.6, 130.6, 106.4, 94.0, 68.1, 61.3, 56.1, 39.5. HRMS (ESI) $m / z:[\mathrm{M}+\mathrm{H}]^{+}$calcd for $\mathrm{C}_{11} \mathrm{H}_{12} \mathrm{O}_{5}$ 225.0763; found, 225.0759 .

5,7-Dihydroxy-8-methoxychroman-4-one (7). To a solution of 7-hydroxy-5,8-dimethoxy-chroman-4-one (28) (30 mg, $0.13 \mathrm{mmol}$ ) in $\mathrm{CH}_{2} \mathrm{Cl}_{2}(3 \mathrm{~mL})$ was added boron trichloride $\left(0.4 \mathrm{~mL}, 1.0 \mathrm{M}\right.$ solution in $\left.\mathrm{CH}_{2} \mathrm{Cl}_{2}\right)$ at $-78{ }^{\circ} \mathrm{C}$. After stirring for $1 \mathrm{~h}$, the reaction mixture was diluted with $\mathrm{CH}_{2} \mathrm{Cl}_{2}$, washed with water, dried over anhydrous $\mathrm{Na}_{2} \mathrm{SO}_{4}$, and concentrated under reduced pressure. The residue was purified by flash column chromatography on silica gel (ethyl acetate $/ n$-hexane $=1: 3)$ to afford 5,7-dihydroxy-8-methoxychroman-4-one (7) (27 mg, 96\%). ${ }^{1} \mathrm{H}$ NMR (600 MHz, dimethyl sulfoxide (DMSO)- $\left.d_{6}\right) \delta 11.95(\mathrm{~s}, 1 \mathrm{H}), 10.64(\mathrm{~s}$, $1 \mathrm{H}), 5.93(\mathrm{~s}, 1 \mathrm{H}), 4.49(\mathrm{t}, 2 \mathrm{H}, J=6.4 \mathrm{~Hz}), 3.62(\mathrm{~s}, 3 \mathrm{H}), 2.77$ $(\mathrm{t}, 2 \mathrm{H}, J=6.4 \mathrm{~Hz}) .{ }^{13} \mathrm{C}\left\{{ }^{1} \mathrm{H}\right\} \mathrm{NMR}\left(150 \mathrm{MHz}, \mathrm{DMSO}-d_{6}\right) \delta$ 196.4, 159.8, 158.7, 154.6, 128.3, 102.1, 95.7, 66.7, 60.4, 35.9. HRMS (ESI) $m / z:[\mathrm{M}+\mathrm{H}]^{+}$calcd for $\mathrm{C}_{10} \mathrm{H}_{10} \mathrm{O}_{5}$ 211.0606; found, 211.0601 .

7-(Benzyloxy)-5,8-dimethoxychroman-4-one. To an acetone $(10 \mathrm{~mL})$ solution of 7-hydroxy-5,8-dimethoxychroman-4-one (28) ( $0.21 \mathrm{~g}, 0.94 \mathrm{mmol})$, benzyl bromide $(0.1 \mathrm{~mL}$, $1.0 \mathrm{mmol})$ and $\mathrm{K}_{2} \mathrm{CO}_{3}(0.39 \mathrm{~g}, 2.8 \mathrm{mmol})$ were added. The reaction mixture was refluxed for $48 \mathrm{~h}$. After cooling to ambient temperature, the reaction mixture was diluted with ethyl acetate and the organic phase was washed with water and saturated $\mathrm{NH}_{4} \mathrm{Cl}$ solution, dried over anhydrous $\mathrm{Na}_{2} \mathrm{SO}_{4}$, and concentrated under reduced pressure. The residue was purified by flash column chromatography on silica gel (ethyl acetate/ $n$ hexane $=1: 1)$ to afford 7-(benzyloxy)-5,8-dimethoxychroman4-one (0.25 g, 85\%). ${ }^{1} \mathrm{H}$ NMR (600 MHz, $\left.\mathrm{CDCl}_{3}\right) \delta 7.42(\mathrm{~d}$, $2 \mathrm{H}, J=7.3 \mathrm{~Hz}), 7.38(\mathrm{~m}, 2 \mathrm{H}), 7.32(\mathrm{~m}, 1 \mathrm{H}), 6.12(\mathrm{~s}, 1 \mathrm{H})$, $5.20(\mathrm{~s}, 2 \mathrm{H}), 4.50(\mathrm{t}, 2 \mathrm{H}, J=6.4 \mathrm{~Hz}), 3.81(\mathrm{~s}, 3 \mathrm{H}), 3.79(\mathrm{~s}$, $3 \mathrm{H}), 2.71(\mathrm{t}, 2 \mathrm{H}, J=6.4 \mathrm{~Hz}) \cdot{ }^{13} \mathrm{C}\left\{{ }^{1} \mathrm{H}\right\} \operatorname{NMR}(150 \mathrm{MHz}$, $\left.\mathrm{CDCl}_{3}\right) \delta 189.4,157.8,157.7,156.8,136.2,131.3,128.8$, $128.4,127.3,106.9,91.2,70.9,67.2,61.3,56.2,38.9$. HRMS (ESI) $\mathrm{m} / z:[\mathrm{M}+\mathrm{H}]^{+}$calcd for $\mathrm{C}_{18} \mathrm{H}_{18} \mathrm{O}_{5}$ 315.1232; found, 315.1230 .

(E)-7-(Benzyloxy)-3-(4'-(benzyloxy)benzylidene)-5,8dimethoxychroman-4-one (29a). To a solution of 7(benzyloxy)-5,8-dimethoxychroman-4-one $(0.10 \mathrm{~g}, 0.32$ $\mathrm{mmol})$ in toluene $(8 \mathrm{~mL})$ were added 4-benzyloxybenzaldehyde $(0.11 \mathrm{~g}, 0.49 \mathrm{mmol})$ and $p$-toluenesulfonic acid $(5.5 \mathrm{mg}$, $0.03 \mathrm{mmol}$ ) at $0{ }^{\circ} \mathrm{C}$. The reaction mixture was refluxed for 12 h. After cooling to ambient temperature, the mixture was concentrated under reduced pressure. The residue was purified by flash column chromatography on silica gel (ethyl acetate/ 
$\left.\mathrm{CH}_{2} \mathrm{Cl}_{2}=1: 3\right)$ to afford (E)-7-(benzyloxy)-3-(4'-(benzyloxy)benzylidene)-5,8-dimethoxychroman-4-one (29a) (69 mg, 43\%). ${ }^{1} \mathrm{H}$ NMR $\left(600 \mathrm{MHz}, \mathrm{CDCl}_{3}\right) \delta 7.78(\mathrm{~s}, 1 \mathrm{H}), 7.44(\mathrm{~d}$, $4 \mathrm{H}, J=7.8 \mathrm{~Hz}), 7.40-7.39(\mathrm{~m}, 4 \mathrm{H}), 7.34-7.33(\mathrm{~m}, 2 \mathrm{H}), 7.25$ $(\mathrm{d}, 2 \mathrm{H}, J=8.7 \mathrm{~Hz}), 7.02(\mathrm{~d}, 2 \mathrm{H}, J=8.7 \mathrm{~Hz}), 6.20(\mathrm{~s}, 1 \mathrm{H})$, $5.31(\mathrm{~d}, 2 \mathrm{H}, J=1.6 \mathrm{~Hz}), 5.23(\mathrm{~s}, 2 \mathrm{H}), 5.11(\mathrm{~s}, 2 \mathrm{H}), 3.84(\mathrm{~s}$, $3 \mathrm{H}), 3.81(\mathrm{~s}, 3 \mathrm{H}) .{ }^{13} \mathrm{C}\left\{{ }^{1} \mathrm{H}\right\} \mathrm{NMR}\left(150 \mathrm{MHz}, \mathrm{CDCl}_{3}\right) \delta 180.0$, $159.7,158.2$, 157.6, 156.2, 136.6, 136.3, 136.1, 131.9, 131.6, $130.2,128.9,128.8,128.4,128.3,127.7,127.6,127.4,115.2$, 108.1, 92.2, 71.1, 70.2, 68.1, 61.5, 56.4. HRMS (ESI) $m / z$ : [M $+\mathrm{H}]^{+}$calcd for $\mathrm{C}_{32} \mathrm{H}_{28} \mathrm{O}_{6}$ 509.1964; found, 509.1961.

( $E$ ) - 7 - ( B enzyloxy ) - 5,8-dimet hoxy-3- ( $4^{\prime}$ methoxybenzylidene)chroman-4-one (29b). To a solution of 7-(benzyloxy)-5,8-dimethoxychroman-4-one (50 mg, $0.16 \mathrm{mmol})$ in toluene $(5 \mathrm{~mL})$ were added 4-methoxybenzaldehyde $(0.03 \mathrm{~mL}, 0.24 \mathrm{mmol})$ and $p$-toluenesulfonic acid $(2.8$ $\mathrm{mg}, 0.02 \mathrm{mmol}$ ) at $0{ }^{\circ} \mathrm{C}$. The reaction mixture was refluxed for $12 \mathrm{~h}$. After cooling to ambient temperature, the mixture was concentrated under reduced pressure. The residue was purified by flash column chromatography on silica gel (ethyl acetate/ $\left.\mathrm{CH}_{2} \mathrm{Cl}_{2}=1: 5\right)$ to afford (E)-7-(benzyloxy)-5,8-dimethoxy-3(4'-methoxybenzylidene)chroman-4-one (29b) (34 mg, 49\%). ${ }^{1} \mathrm{H}$ NMR $\left(600 \mathrm{MHz}, \mathrm{CDCl}_{3}\right) \delta 7.78(\mathrm{~s}, 1 \mathrm{H}), 7.44(\mathrm{~d}, 2 \mathrm{H}, J=$ $7.3 \mathrm{~Hz}$ ), 7.41-7.39 (m, 2H), 7.35-7.33 (m, 1H), 7.26-7.25 $(\mathrm{m}, 2 \mathrm{H}), 6.95(\mathrm{~d}, 2 \mathrm{H}, J=8.7 \mathrm{~Hz}), 6.20(\mathrm{~s}, 1 \mathrm{H}), 5.31(\mathrm{~d}, 1 \mathrm{H}, J$ $=1.7 \mathrm{~Hz}), 5.23(\mathrm{~s}, 1 \mathrm{H}), 3.85(\mathrm{~s}, 1 \mathrm{H}), 3.85(\mathrm{~s}, 1 \mathrm{H}), 3.81(\mathrm{~s}$, 1H). ${ }^{13} \mathrm{C}\left\{{ }^{1} \mathrm{H}\right\}$ NMR $\left(150 \mathrm{MHz}, \mathrm{CDCl}_{3}\right) \delta 180.1,160.6,158.2$, $157.5,156.2,136.3,136.2,131.9,131.6,130.1,128.9,128.4$, 127.5, 127.4, 114.3, 108.1, 92.3, 71.1, 68.1, 61.5, 56.4, 55.5. HRMS (ESI) $m / z$ : $[\mathrm{M}+\mathrm{H}]^{+}$calcd for $\mathrm{C}_{26} \mathrm{H}_{24} \mathrm{O}_{6} 433.1651$; found, 433.1649 .

(E) - 7-(Benzyloxy)-3-( $3^{\prime}, 4^{\prime}$ - bis (benzyloxy)benzylidene)-5,8-dimethoxychroman-4-one (29c). To a solution of 7-(benzyloxy)-5,8-dimethoxychroman-4-one (0.10 g, $0.32 \mathrm{mmol})$ in toluene $(8 \mathrm{~mL})$ were added $3,4-$ bis(benzyloxy)benzaldehyde $(0.15 \mathrm{~g}, 0.48 \mathrm{mmol})$ and $p$ toluenesulfonic acid $(6.2 \mathrm{mg}, 0.04 \mathrm{mmol})$ at $0{ }^{\circ} \mathrm{C}$. The reaction mixture was refluxed for $13 \mathrm{~h}$. After cooling to ambient temperature, the mixture was concentrated under reduced pressure. The residue was purified by flash column chromatography on silica gel (ethyl acetate $/ n$-hexane $/ \mathrm{CH}_{2} \mathrm{Cl}_{2}$ $=1: 3: 0.1)$ to afford (E)-7-(benzyloxy)-3-( $3^{\prime}, 4^{\prime}$-bis(benzyloxy)benzylidene)-5,8-dimethoxychroman-4-one (29c) (0.10 g, $51 \%) .{ }^{1} \mathrm{H} \mathrm{NMR}\left(600 \mathrm{MHz}, \mathrm{CDCl}_{3}\right) \delta 7.69(\mathrm{~s}, 1 \mathrm{H}), 7.46-$ $7.43(\mathrm{~m}, 6 \mathrm{H}), 7.42-7.37(\mathrm{~m}, 6 \mathrm{H}), 7.34-7.30(\mathrm{~m}, 3 \mathrm{H}), 6.95$ $(\mathrm{d}, 1 \mathrm{H}, J=8.8 \mathrm{~Hz}), 6.84-6.82(\mathrm{~m}, 2 \mathrm{H}), 6.19(\mathrm{~s}, 1 \mathrm{H}), 5.22(\mathrm{~s}$, $2 \mathrm{H}), 5.21(\mathrm{~s}, 2 \mathrm{H}) 5.18(\mathrm{~s}, 2 \mathrm{H}), 5.15(\mathrm{~d}, 2 \mathrm{H}, J=1.6 \mathrm{~Hz}), 3.83$ (s, 3H), $3.82(\mathrm{~s}, 3 \mathrm{H}) \cdot{ }^{13} \mathrm{C}\left\{{ }^{1} \mathrm{H}\right\} \mathrm{NMR}\left(150 \mathrm{MHz}, \mathrm{CDCl}_{3}\right) \delta$ $179.8,158.1,157.5,156.1,150.1,148.6,137.0,136.8,136.3$, $136.1,131.5,130.4,128.8,128.7,128.7,128.4,128.1,128.1$, 127.3, 127.3, 124.2, 116.9, 114.3, 107.9, 92.2, 71.5, 71.1, 71.0, 67.9, 61.4, 56.3. HRMS (ESI) $m / z:[\mathrm{M}+\mathrm{H}]^{+}$calcd for $\mathrm{C}_{39} \mathrm{H}_{34} \mathrm{O}_{7}$ 615.2383; found, 615.2379.

(E)-7-(Benzyloxy)-3-(3'-(benzyloxy)-4' -methoxybenzylidene)-5,8-dimethoxychroman-4-one (29d). To a solution of 7-(benzyloxy)-5,8-dimethoxychroman-4-one (0.27 g, $0.87 \mathrm{mmol}$ ) in toluene $(15 \mathrm{~mL})$ were added 3-(benzyloxy)4-methoxybenzaldehyde $(0.32 \mathrm{mg}, 1.3 \mathrm{mmol})$ and $p$ toluenesulfonic acid (14 mg, $0.09 \mathrm{mmol})$ at $0{ }^{\circ} \mathrm{C}$. The reaction mixture was refluxed for $13 \mathrm{~h}$. After cooling to ambient temperature, the mixture was concentrated under reduced pressure. The residue was purified by flash column chromatography on silica gel (ethyl acetate $/ n$-hexane $/ \mathrm{CH}_{2} \mathrm{Cl}_{2}$
$=1: 1: 0.1)$ to afford $(E)-7$-(benzyloxy)-3-(3'-(benzyloxy) $-4^{\prime}$ methoxybenzylidene)-5,8-dimethoxychroman-4-one (29d) $(0.26 \mathrm{~g}, 56 \%) .{ }^{1} \mathrm{H}$ NMR $\left(600 \mathrm{MHz}, \mathrm{CDCl}_{3}\right) \delta 7.69(\mathrm{~s}, 1 \mathrm{H})$, 7.46-7.43 (m, 4H), 7.41-7.38 (m, 4H), 7.36-7.30 (m, 2H), $6.93(\mathrm{~d}, 1 \mathrm{H}, J=8.4 \mathrm{~Hz}), 6.89(\mathrm{dd}, 1 \mathrm{H}, J=8.4,1.8 \mathrm{~Hz}), 6.79$ $(\mathrm{d}, 1 \mathrm{H}, J=1.8 \mathrm{~Hz}), 6.19(\mathrm{~s}, 1 \mathrm{H}), 5.23(\mathrm{~s}, 2 \mathrm{H}), 5.18(\mathrm{~s}, 2 \mathrm{H})$, $5.14(\mathrm{~d}, 2 \mathrm{H}, J=1.7 \mathrm{~Hz}), 3.94(\mathrm{~s}, 3 \mathrm{H}), 3.84(\mathrm{~s}, 3 \mathrm{H}), 3.82(\mathrm{~s}$, $3 \mathrm{H}) .{ }^{13} \mathrm{C}\left\{{ }^{1} \mathrm{H}\right\}$ NMR $\left(150 \mathrm{MHz}, \mathrm{CDCl}_{3}\right) \delta 179.9,158.2,157.5$, $156.2,150.9,148.0,136.9,136.3,136.3,131.6,130.3,128.9$, $128.8,128.4,128.2,127.6,127.4,127.3,124.2,115.9,111.6$, 108.0, 92.2, 71.3, 71.1, 67.9, 61.5, 56.4, 56.2. HRMS (ESI) $m /$ $z:[\mathrm{M}+\mathrm{H}]^{+}$calcd for $\mathrm{C}_{33} \mathrm{H}_{30} \mathrm{O}_{7}$ 539.2070; found, 539.2071.

Punctatin (8). To a solution of (E)-7-(benzyloxy)-3-(4'(benzyloxy)benzylidene)-5,8-dimethoxychroman-4-one (29a) (30 mg, $0.06 \mathrm{mmol})$ in $\mathrm{CH}_{2} \mathrm{Cl}_{2}(2 \mathrm{~mL})$ was added boron trichloride $\left(0.18 \mathrm{~mL}, 1.0 \mathrm{M}\right.$ solution in $\left.\mathrm{CH}_{2} \mathrm{Cl}_{2}\right)$ at $-78{ }^{\circ} \mathrm{C}$. After stirring for $1 \mathrm{~h}$, the reaction mixture was diluted with $\mathrm{CH}_{2} \mathrm{Cl}_{2}$, washed with water, dried over anhydrous $\mathrm{Na}_{2} \mathrm{SO}_{4}$, and concentrated under reduced pressure. The residue was purified by flash column chromatography on silica gel (ethyl acetate $\left./ \mathrm{CH}_{2} \mathrm{Cl}_{2}=1: 3\right)$ to afford punctatin (8) $(9.1 \mathrm{mg}, 49 \%)$. ${ }^{1} \mathrm{H}$ NMR (600 MHz, DMSO- $\left.d_{6}\right) \delta 12.62(\mathrm{~s}, 1 \mathrm{H}), 10.74$ (br s, $1 \mathrm{H}), 10.17$ (br s, $1 \mathrm{H}), 7.69(\mathrm{~s}, 1 \mathrm{H}), 7.35$ (d, $2 \mathrm{H}, J=8.6 \mathrm{~Hz})$, $6.89(\mathrm{~d}, 2 \mathrm{H}, J=8.6 \mathrm{~Hz}), 5.99(\mathrm{~s}, 1 \mathrm{H}), 5.41(\mathrm{~d}, 3 \mathrm{H}, J=1.4$ $\mathrm{Hz}), 3.63(\mathrm{~s}, 3 \mathrm{H}) .{ }^{13} \mathrm{C}\left\{{ }^{1} \mathrm{H}\right\} \mathrm{NMR}\left(150 \mathrm{MHz}, \mathrm{DMSO}-d_{6}\right) \delta$ $184.6,160.1,159.5,159.5,153.4,136.9,132.9,128.3,126.1$, 124.7, 115.8, 101.8, 96.2, 67.4, 60.5. HRMS (ESI) $m / z:[\mathrm{M}+$ $\mathrm{H}]^{+}$calcd for $\mathrm{C}_{17} \mathrm{H}_{14} \mathrm{O}_{6} 315.0869$; found, 315.0865 .

4'-O-Methylpunctatin (9). To a solution of (E)-7(benzyloxy)-5,8-dimethoxy-3-(4-methoxybenzylidene)-chroman-4-one (29b) (34 mg, $0.08 \mathrm{mmol})$ in $\mathrm{CH}_{2} \mathrm{Cl}_{2}(2 \mathrm{~mL})$ was added boron trichloride $\left(0.25 \mathrm{~mL}, 1.0 \mathrm{M}\right.$ solution in $\left.\mathrm{CH}_{2} \mathrm{Cl}_{2}\right)$ at $-78{ }^{\circ} \mathrm{C}$. After stirring for $1 \mathrm{~h}$, the reaction mixture was diluted with $\mathrm{CH}_{2} \mathrm{Cl}_{2}$, washed with water, dried over anhydrous $\mathrm{Na}_{2} \mathrm{SO}_{4}$, and concentrated under reduced pressure. The residue was purified by flash column chromatography on silica gel (ethyl acetate $/ \mathrm{CH}_{2} \mathrm{Cl}_{2}=1: 3$ ) to afford $4^{\prime}$-O-methylpunctatin (9) (11 mg, 42\%). ${ }^{1} \mathrm{H}$ NMR (600 MHz, DMSO-d $\left.d_{6}\right) \delta$ $12.58(\mathrm{~s}, 1 \mathrm{H}), 10.77$ (br s, $1 \mathrm{H}), 7.73(\mathrm{~s}, 1 \mathrm{H}), 7.45$ (d, $2 \mathrm{H}, J=$ $8.7 \mathrm{~Hz}), 7.06$ (d, $2 \mathrm{H}, J=8.8 \mathrm{~Hz}), 5.99(\mathrm{~s}, 1 \mathrm{H}), 5.41(\mathrm{~d}, 2 \mathrm{H}, J$ $=1.8 \mathrm{~Hz}), 3.83(\mathrm{~s}, 3 \mathrm{H}), 3.63(\mathrm{~s}, 3 \mathrm{H}) .{ }^{13} \mathrm{C}\left\{{ }^{1} \mathrm{H}\right\} \mathrm{NMR}(150$ $\left.\mathrm{MHz}, \mathrm{DMSO}-d_{6}\right) \delta 184.6,160.7,160.2,159.6,153.4,136.4$, 132.6, 128.4, 127.1, 126.2, 114.4, 101.9, 96.2, 67.4, 60.5, 55.4. HRMS (ESI) $m / z:[\mathrm{M}+\mathrm{H}]^{+}$calcd for $\mathrm{C}_{18} \mathrm{H}_{16} \mathrm{O}_{6}$ 329.1025; found, 329.1025 .

3-(3', 4'-Dihydroxybenzylidene)-5,7-dihydroxy-8-methoxychroman-4-one (10). To a solution of (E)-7(benzyloxy)-3-(3,4-bis(benzyloxy)benzylidene)-5,8-dimethoxy-chroman-4-one (29c) (45 mg, $0.07 \mathrm{mmol}$ ) in $\mathrm{CH}_{2} \mathrm{Cl}_{2}$ (2 $\mathrm{mL})$ was added boron trichloride $(0.37 \mathrm{~mL}, 1.0 \mathrm{M}$ solution in $\mathrm{CH}_{2} \mathrm{Cl}_{2}$ ) at $-78^{\circ} \mathrm{C}$. After stirring for $3 \mathrm{~h}$, the reaction mixture was diluted with $\mathrm{CH}_{2} \mathrm{Cl}_{2}$, washed with water, dried over $\mathrm{Na}_{2} \mathrm{SO}_{4}$, and concentrated under reduced pressure. The residue was purified by flash column chromatography on silica gel (ethyl acetate $\left./ \mathrm{CH}_{2} \mathrm{Cl}_{2}=1: 3\right)$ to afford $3-\left(3^{\prime}, 4^{\prime}\right.$ dihydroxybenzylidene)-5,7-dihydroxy-8-methoxychroman-4one (10) (14 mg, 60\%). ${ }^{1} \mathrm{H}$ NMR (600 MHz, CD $\left.\mathrm{CD}_{3} \mathrm{OD}\right) \delta$ $12.58(\mathrm{~s}, 1 \mathrm{H}), 7.69(\mathrm{~s}, 1 \mathrm{H}), 6.87(\mathrm{~d}, 1 \mathrm{H}, J=8.2 \mathrm{~Hz}), 6.84(\mathrm{~d}$, $1 \mathrm{H}, J=2.0 \mathrm{~Hz}), 6.78(\mathrm{dd}, 1 \mathrm{H}, J=8.2,2.0 \mathrm{~Hz}), 5.96(\mathrm{~s}, 1 \mathrm{H})$, $5.39(\mathrm{~d}, 2 \mathrm{H}, J=1.7 \mathrm{~Hz}), 3.74(\mathrm{~s}, 3 \mathrm{H}) .{ }^{13} \mathrm{C}\left\{{ }^{1} \mathrm{H}\right\} \operatorname{NMR}(150$ $\left.\mathrm{MHz}, \mathrm{CD}_{3} \mathrm{OD}\right) \delta 186.6,161.6,161.2,154.9,149.1,146.7$, 138.8 , 129.7, 128.0, 127.5, 124.8, 118.4, 116.6, 103.6, 97.3, 
69.0, 61.6. HRMS (ESI) $m / z$ : $[\mathrm{M}+\mathrm{H}]^{+}$calcd for $\mathrm{C}_{17} \mathrm{H}_{14} \mathrm{O}_{7}$ 331.0818; found, 331.0813 .

7-Hydroxy-3-(4'-hydroxybenzyl)-5,8-dimethoxychroman-4-one (30). (E)-7-(Benzyloxy)-3-(4-(benzyloxy)benzylidene)-5,8-dimethoxychroman-4-one (29a) (21 mg, $0.04 \mathrm{mmol})$ and $10 \% \mathrm{Pd} / \mathrm{C}(1.5 \mathrm{mg}, 0.01 \mathrm{mmol})$ in methanol/dichloromethane $(1 \mathrm{~mL}, 1 \mathrm{~mL})$ were placed under an atmosphere of hydrogen. After stirring for $3 \mathrm{~h}$, the mixture was filtered through a Celite pad. After the filtrate was concentrated under reduced pressure, purification of the residue via flash column chromatography on silica gel (ethyl acetate $/ n$-hexane $=1: 1)$ afforded 7-hydroxy-3-(4'-hydroxybenzyl)-5,8-dimethoxychroman-4-one (30) (12 mg, 91\%). ${ }^{1} \mathrm{H}$ NMR $\left(600 \mathrm{MHz}, \mathrm{CD}_{3} \mathrm{OD}\right) \delta 7.05(\mathrm{~d}, 2 \mathrm{H}, J=8.4 \mathrm{~Hz}), 6.73(\mathrm{~d}$, $2 \mathrm{H}, J=8.4 \mathrm{~Hz}), 6.14(\mathrm{~s}, 1 \mathrm{H}), 4.31(\mathrm{dd}, 1 \mathrm{H}, J=11.3,3.9 \mathrm{~Hz})$, $4.16(\mathrm{dd}, 1 \mathrm{H}, J=11.3,6.6 \mathrm{~Hz}), 3.79(\mathrm{~s}, 3 \mathrm{H}), 3.74(\mathrm{~s}, 3 \mathrm{H}), 3.03$ (dd, $1 \mathrm{H}, J=13.3,4.2 \mathrm{~Hz}$ ), 2.70-2.66 (m, 1H), 2.63 (dd, $1 \mathrm{H}, J$ $=13.3,10.2 \mathrm{~Hz}) .{ }^{13} \mathrm{C}\left\{{ }^{1} \mathrm{H}\right\} \mathrm{NMR}\left(150 \mathrm{MHz}, \mathrm{CD}_{3} \mathrm{OD}\right) \delta 194.0$, 159.7, 159.0, 158.1, 157.1, 131.2, 130.4, 116.4, 105.6, 94.1, 70.2, 61.4, 56.2, 49.8, 33.4. HRMS (ESI) $m / z:[\mathrm{M}+\mathrm{H}]^{+}$calcd for $\mathrm{C}_{18} \mathrm{H}_{18} \mathrm{O}_{6} 331.1181$; found, 331.1181 .

7-Hydroxy-3-(3'-hydroxy-4' -methoxybenzyl)-5,8-dimethoxychroman-4-one (31). (E)-7-(Benzyloxy)-3-(3(benzyloxy)-4-methoxybenzylidene)-5,8-dimethoxychroman4-one (29d) $(0.10 \mathrm{mg}, 0.19 \mathrm{mmol})$ and $10 \% \mathrm{Pd} / \mathrm{C}(6.0 \mathrm{mg}$, $0.06 \mathrm{mmol})$ in methanol/dichloromethane $(3 \mathrm{~mL}, 3 \mathrm{~mL})$ were placed under an atmosphere of hydrogen. After stirring for $1 \mathrm{~h}$, the mixture was filtered through a Celite pad. After the filtrate was concentrated under reduced pressure, purification of the residue via flash column chromatography on silica gel (ethyl acetate $/ n$-hexane $=1: 1)$ afforded 7-hydroxy-3-( $3^{\prime}$-hydroxy-4' methoxybenzyl)-5,8-dimethoxychroman-4-one (31) (67 mg, 98\%). ${ }^{1} \mathrm{H}$ NMR (600 MHz, CD 3 OD) $\delta 6.85$ (d, $1 \mathrm{H}, J=8.2$ $\mathrm{Hz}), 6.71(\mathrm{~d}, 1 \mathrm{H}, J=2.0 \mathrm{~Hz}), 6.67(\mathrm{dd}, 1 \mathrm{H}, J=8.2,2.0 \mathrm{~Hz})$, $6.14(\mathrm{~s}, 1 \mathrm{H}), 4.31(\mathrm{dd}, 1 \mathrm{H}, J=11.3,4.0 \mathrm{~Hz}), 4.16(\mathrm{dd}, 1 \mathrm{H}, J=$ 11.3, $6.8 \mathrm{~Hz}), 3.82(\mathrm{~s}, 3 \mathrm{H}), 3.79(\mathrm{~s}, 3 \mathrm{H}), 3.74(\mathrm{~s}, 3 \mathrm{H}), 3.01$ (dd, $1 \mathrm{H}, J=13.7,4.6 \mathrm{~Hz}), 2.71-2.67(\mathrm{~m}, 1 \mathrm{H}), 2.60(\mathrm{dd}, 1 \mathrm{H}, J$ $=13.7,10.3 \mathrm{~Hz}) .{ }^{13} \mathrm{C}\left\{{ }^{1} \mathrm{H}\right\} \operatorname{NMR}\left(150 \mathrm{MHz}, \mathrm{CD}_{3} \mathrm{OD}\right) \delta 193.9$, $159.7,159.1,158.1,147.8,147.7,132.6,130.4,121.4,117.1$, 112.9, 105.6, 94.1, 70.2, 61.4, 56.4, 56.2, 49.7, 33.6. HRMS (ESI) $m / z:[\mathrm{M}+\mathrm{H}]^{+}$calcd for $\mathrm{C}_{19} \mathrm{H}_{20} \mathrm{O}_{7} 361.1287$; found, 361.1284 .

(E)-3-(4'-Hydroxybenzylidene)-5,7,8-trimethoxychroman-4-one (32a). ${ }^{1} \mathrm{H}$ NMR $\left(600 \mathrm{MHz}, \mathrm{CDCl}_{3}\right) \delta 7.79$ (s, $1 \mathrm{H}), 7.19$ (d, 2H, $J=8.3 \mathrm{~Hz}), 6.94(\mathrm{~d}, 2 \mathrm{H}, J=8.3 \mathrm{~Hz}), 6.45$ $(\mathrm{s}, 1 \mathrm{H}), 6.17(\mathrm{~s}, 1 \mathrm{H}), 5.30(\mathrm{~s}, 2 \mathrm{H}), 3.95(\mathrm{~s}, 3 \mathrm{H}), 3.92(\mathrm{~s}, 3 \mathrm{H})$, $3.79(\mathrm{~s}, 3 \mathrm{H}) .{ }^{13} \mathrm{C}\left\{{ }^{1} \mathrm{H}\right\} \mathrm{NMR}\left(150 \mathrm{MHz}, \mathrm{CDCl}_{3}\right) \delta 180.4$, $158.7,158.7,157.5,156.1,136.8,132.1,131.0,129.7,127.1$, 116.0, 107.6, 90.1, 68.0, 61.5, 56.4, 56.2.

(E)-7-Hydroxy-3-(4'-hydroxybenzylidene)-5,8-dimethoxychroman-4-one (32b). ${ }^{1} \mathrm{H}$ NMR (600 MHz, $\left.\mathrm{CD}_{3} \mathrm{OD}\right)$ $\delta 7.71(\mathrm{~s}, 1 \mathrm{H}), 7.24(\mathrm{~d}, 2 \mathrm{H}, J=8.5 \mathrm{~Hz}), 6.88(\mathrm{~d}, 2 \mathrm{H}, J=8.5$ $\mathrm{Hz}), 6.20(\mathrm{~s}, 1 \mathrm{H}), 5.30(\mathrm{~d}, 2 \mathrm{H}, J=1.7 \mathrm{~Hz}), 3.82(\mathrm{~s}, 3 \mathrm{H}), 3.75$ $(\mathrm{s}, 3 \mathrm{H}) .{ }^{13} \mathrm{C}\left\{{ }^{1} \mathrm{H}\right\}$ NMR $\left(150 \mathrm{MHz}, \mathrm{CD}_{3} \mathrm{OD}\right) \delta 180.4,159.0$, $158.5,157.6,156.3,136.3,131.9,129.4,129.2,125.8,115.3$, 106.0, 93.4, 67.5, 60.2, 54.8.

( $E$ ) - 7 - H y d r ox y - 5, 8 - d i m e t h ox y - 3- ( $4^{\prime}$ methoxybenzylidene)chroman-4-one (32c). ${ }^{1} \mathrm{H}$ NMR $\left(600 \mathrm{MHz}, \mathrm{DMSO}-d_{6}\right) \delta 10.39(\mathrm{~s}, 1 \mathrm{H}), 7.55(\mathrm{~s}, 1 \mathrm{H}), 7.38$ $(\mathrm{d}, 2 \mathrm{H}, J=8.8 \mathrm{~Hz}), 7.03(\mathrm{~d}, 2 \mathrm{H}, J=8.8 \mathrm{~Hz}), 6.20(\mathrm{~s}, 1 \mathrm{H})$, 5.27 (d, $2 \mathrm{H}, J=1.6 \mathrm{~Hz}), 3.82(\mathrm{~s}, 3 \mathrm{H}), 3.73(\mathrm{~s}, 3 \mathrm{H}), 3.63(\mathrm{~s}$, $3 \mathrm{H}) .{ }^{13} \mathrm{C}\left\{{ }^{1} \mathrm{H}\right\}$ NMR $\left(150 \mathrm{MHz}, \mathrm{DMSO}-d_{6}\right) \delta 177.9,160.1$,
$157.5,156.8,155.9,134.1,132.0,130.3,129.3,126.7,114.3$, $106.1,94.2,67.5,60.6,55.7,55.3$.

3-(4' -(Benzyloxy)benzyl)-5-hydroxy-7,8-dimethoxychroman-4-one (33a). ${ }^{1} \mathrm{H} \mathrm{NMR}\left(600 \mathrm{MHz}, \mathrm{CDCl}_{3}\right) \delta 12.06$ (s, $1 \mathrm{H}), 7.44(\mathrm{~d}, 2 \mathrm{H}, J=7.5 \mathrm{~Hz}), 7.39(\mathrm{t}, 2 \mathrm{H}, J=7.5 \mathrm{~Hz}), 7.33$ $(\mathrm{t}, 1 \mathrm{H}, J=7.3 \mathrm{~Hz}), 7.16(\mathrm{~d}, 2 \mathrm{H}, J=8.6 \mathrm{~Hz}), 6.94(\mathrm{~d}, 2 \mathrm{H}, J=$ $8.6 \mathrm{~Hz}), 6.11(\mathrm{~s}, 1 \mathrm{H}), 5.06(\mathrm{~s}, 2 \mathrm{H}), 4.36(\mathrm{dd}, 1 \mathrm{H}, \mathrm{J}=11.5,4.2$ $\mathrm{Hz}), 4.21(\mathrm{dd}, 1 \mathrm{H}, J=11.5,7.1 \mathrm{~Hz}), 3.89(\mathrm{~s}, 3 \mathrm{H}), 3.78(\mathrm{~s}$, $3 \mathrm{H}), 3.18(\mathrm{dd}, 1 \mathrm{H}, J=14.0,4.5 \mathrm{~Hz}), 2.85(\mathrm{~m}, 1 \mathrm{H}), 2.74(\mathrm{dd}$, $1 \mathrm{H}, J=14.0,10.3 \mathrm{~Hz}) \cdot{ }^{13} \mathrm{C}\left\{{ }^{1} \mathrm{H}\right\} \mathrm{NMR}\left(150 \mathrm{MHz}, \mathrm{CDCl}_{3}\right) \delta$ $198.3,161.4,160.4,157.8,153.8,137.1,130.3,130.1,129.5$, $128.7,128.1,127.6,115.2,102.5,93.1,70.2,69.5,61.5,56.4$, 47.1, 32.1 .

3-(3', 4'-Bis(benzyloxy)benzyl)-5-hydroxy-7,8-dimethoxychroman-4-one (33b). ${ }^{1} \mathrm{H}$ NMR (600 $\mathrm{MHz}, \mathrm{CDCl}_{3}$ ) $\delta 12.04(\mathrm{~s}, 1 \mathrm{H}), 7.44(\mathrm{~d}, 4 \mathrm{H}, J=7.7 \mathrm{~Hz}), 7.36(\mathrm{td}, 4 \mathrm{H}, J=7.4$, $4.1 \mathrm{~Hz}), 7.30(\mathrm{dd}, 2 \mathrm{H}, J=14.3,7.1 \mathrm{~Hz}), 6.88(\mathrm{~d}, 1 \mathrm{H}, J=8.2$ $\mathrm{Hz}), 6.80(\mathrm{~d}, 1 \mathrm{H}, J=2.0 \mathrm{~Hz}), 6.74(\mathrm{dd}, 1 \mathrm{H}, J=8.2,2.0 \mathrm{~Hz})$, $6.10(\mathrm{~s}, 1 \mathrm{H}), 5.15(\mathrm{~d}, 4 \mathrm{H}, J=4.9 \mathrm{~Hz}), 4.27(\mathrm{dd}, 1 \mathrm{H}, J=11.5$, $4.2 \mathrm{~Hz}$ ), 4.09 (dd, $1 \mathrm{H}, J=11.5,7.3 \mathrm{~Hz}), 3.89(\mathrm{~s}, 3 \mathrm{H}), 3.77$ (s, $3 \mathrm{H}), 3.12(\mathrm{dd}, 1 \mathrm{H}, J=14.0,4.4 \mathrm{~Hz}), 2.79(\mathrm{~m}, 1 \mathrm{H}), 2.68(\mathrm{dd}$, $1 \mathrm{H}, J=14.0,10.2 \mathrm{~Hz})$.

3-(3'-(Benzyloxy)-4' -methoxybenzyl)-5-hydroxy-7,8dimethoxychroman-4-one (33c). ${ }^{1} \mathrm{H}$ NMR (600 MHz, $\left.\mathrm{CDCl}_{3}\right) \delta 12.04(\mathrm{~s}, 1 \mathrm{H}), 7.43(\mathrm{~d}, 2 \mathrm{H}, J=7.5 \mathrm{~Hz}), 7.36(\mathrm{t}, 2 \mathrm{H}$, $J=7.6 \mathrm{~Hz}), 7.29(\mathrm{t}, 1 \mathrm{H}, J=7.4 \mathrm{~Hz}), 6.84(\mathrm{~d}, 1 \mathrm{H}, J=8.1 \mathrm{~Hz})$ $6.78(\mathrm{dd}, 1 \mathrm{H}, J=8.1,1.9 \mathrm{~Hz}), 6.75(\mathrm{~d}, 1 \mathrm{H}, J=1.9 \mathrm{~Hz}), 6.10$ (s, 1H), 5.14 (s, 2H), 4.27 (dd, $1 \mathrm{H}, J=11.5,4.2 \mathrm{~Hz}), 4.09$ (dd, $1 \mathrm{H}, J=11.5,7.3 \mathrm{~Hz}), 3.89(\mathrm{~s}, 3 \mathrm{H}), 3.88(\mathrm{~s}, 3 \mathrm{H}), 3.78(\mathrm{~s}, 3 \mathrm{H})$, $3.11(\mathrm{dd}, 1 \mathrm{H}, J=13.9,4.4 \mathrm{~Hz}), 2.78(\mathrm{~m}, 1 \mathrm{H}), 2.69(\mathrm{dd}, 1 \mathrm{H}, J$ $=13.9,10.1 \mathrm{~Hz}) .{ }^{13} \mathrm{C}\left\{{ }^{1} \mathrm{H}\right\} \mathrm{NMR}\left(150 \mathrm{MHz}, \mathrm{CDCl}_{3}\right) \delta 198.2$, $161.5,160.4,153.8,148.8,148.2$, 137.1, 130.2, 129.5, 128.7, $128.0,127.5,122.1,115.4,112.1,102.5,93.1,71.2,69.4,61.5$, $56.4,56.2,46.9,32.4$.

3-(3'-Hydroxy-4' -methoxybenzyl)-5,7,8-trimethoxychroman-4-one (33d). ${ }^{1} \mathrm{H} \mathrm{NMR}\left(600 \mathrm{MHz}^{\mathrm{CDCl}}{ }_{3}\right) \delta 6.81$ $(\mathrm{s}, 1 \mathrm{H}), 6.78(\mathrm{~d}, 1 \mathrm{H}, J=8.1 \mathrm{~Hz}), 6.71(\mathrm{~d}, 1 \mathrm{H}, J=8.1 \mathrm{~Hz})$, $6.12(\mathrm{~s}, 1 \mathrm{H}), 5.59(\mathrm{~s}, 1 \mathrm{H}), 4.35(\mathrm{dd}, 1 \mathrm{H}, J=11.2,3.6 \mathrm{~Hz}), 4.18$ (dd, $1 \mathrm{H}, J=10.8,8.0 \mathrm{~Hz}), 3.94(\mathrm{~s}, 3 \mathrm{H}), 3.92(\mathrm{~s}, 3 \mathrm{H}), 3.87(\mathrm{~s}$, $3 \mathrm{H}), 3.79$ (s, 3H), $3.18(\mathrm{dd}, J=14.0,4.0 \mathrm{~Hz}, 1 \mathrm{H}), 2.78(\mathrm{~m}$, $1 \mathrm{H}), 2.58(\mathrm{dd}, J=14.0,10.5 \mathrm{~Hz}, 1 \mathrm{H}) .{ }^{13} \mathrm{C}\left\{{ }^{1} \mathrm{H}\right\} \operatorname{NMR}(150$ $\left.\mathrm{MHz}, \mathrm{CDCl}_{3}\right) \delta 191.6,158.5,158.2,156.3,145.6,145.3,131.8$, 130.6, 120.6, 115.2, 110.8, 105.7, 89.2, 69.3, 61.2, 56.2, 56.1, $56.0,48.5,32.1$.

3,9-Dihydropunctatin (11). To a solution of 7-hydroxy-3(4'-hydroxybenzyl)-5,8-dimethoxy-chroman-4-one (30) (17 $\mathrm{mg}, \quad 0.05 \mathrm{mmol})$ in $\mathrm{CH}_{2} \mathrm{Cl}_{2}(2 \mathrm{~mL})$ was added boron trichloride $\left(0.15 \mathrm{~mL}, 1.0 \mathrm{M}\right.$ solution in $\left.\mathrm{CH}_{2} \mathrm{Cl}_{2}\right)$ at $-78{ }^{\circ} \mathrm{C}$. After stirring for $2 \mathrm{~h}$, the reaction mixture was diluted with $\mathrm{CH}_{2} \mathrm{Cl}_{2}$, washed with water, dried over anhydrous $\mathrm{Na}_{2} \mathrm{SO}_{4}$, and concentrated under reduced pressure. The residue was purified by flash column chromatography on silica gel (ethyl acetate $/ n$-hexane $=1: 1)$ to afford 3,9-dihydropunctatin (11) $(8.0 \mathrm{mg}, 50 \%) .{ }^{1} \mathrm{H}$ NMR (600 MHz, DMSO-d $\left.d_{6}\right) \delta 11.96(\mathrm{~s}$, $1 \mathrm{H}), 10.68(\mathrm{~s}, 1 \mathrm{H}), 9.26(\mathrm{~s}, 1 \mathrm{H}), 7.03(\mathrm{~d}, 2 \mathrm{H}, J=8.4 \mathrm{~Hz}), 6.69$ $(\mathrm{d}, 2 \mathrm{H}, J=8.4 \mathrm{~Hz}), 5.94(\mathrm{~s}, 1 \mathrm{H}), 4.31(\mathrm{dd}, 1 \mathrm{H}, J=11.3,4.4$ $\mathrm{Hz}), 4.13(\mathrm{dd}, 1 \mathrm{H}, J=11.4,7.9 \mathrm{~Hz}), 3.60(\mathrm{~s}, 3 \mathrm{H}), 3.00(\mathrm{dd}$, $1 \mathrm{H}, J=13.7,5.0 \mathrm{~Hz}), 2.98-2.93(\mathrm{~m}, 1 \mathrm{H}), 2.61(\mathrm{dd}, 1 \mathrm{H}, J=$ 13.7, 9.3 Hz). ${ }^{13} \mathrm{C}\left\{{ }^{1} \mathrm{H}\right\}$ NMR $\left(150 \mathrm{MHz}, \mathrm{DMSO}-d_{6}\right) \delta 198.0$, $159.8,158.8,155.9,154.3,130.0,128.2,128.0,115.2,101.3$, 95.9, 69.1, 60.4, 45.6, 31.2. HRMS (ESI) $\mathrm{m} / z:[\mathrm{M}+\mathrm{H}]^{+}$calcd for $\mathrm{C}_{17} \mathrm{H}_{16} \mathrm{O}_{6} 317.1025$; found, 317.1019 . 
5,6-Dihydroxy-3-(3'-hydroxy-4'-methoxybenzyl)-8methoxychroman-4-one (12). To a solution of 7-hydroxy3-(3'-hydroxy-4'-methoxybenzyl)-5,8-dimethoxy-chroman-4one (31) $(20 \mathrm{mg}, 0.05 \mathrm{mmol})$ in $\mathrm{CH}_{2} \mathrm{Cl}_{2}(2 \mathrm{~mL})$ was added boron trichloride $\left(0.16 \mathrm{~mL}, 1.0 \mathrm{M}\right.$ solution in $\left.\mathrm{CH}_{2} \mathrm{Cl}_{2}\right)$ at -78 ${ }^{\circ} \mathrm{C}$. After stirring for $3 \mathrm{~h}$, the reaction mixture was diluted with $\mathrm{CH}_{2} \mathrm{Cl}_{2}$, washed with water, dried over anhydrous $\mathrm{Na}_{2} \mathrm{SO}_{4}$, and concentrated under reduced pressure. The residue was purified by flash column chromatography on silica gel (ethyl acetate $/ n$-hexane $=1: 1)$ to afford 5,6-dihydroxy-3-( $3^{\prime}$-hydroxy$4^{\prime}$-methoxybenzyl)-8-methoxychroman-4-one (12) (8.1 mg, 42\%). ${ }^{1} \mathrm{H}$ NMR (600 MHz, CD $\left.3 \mathrm{OD}\right) \delta 6.86(\mathrm{~d}, 1 \mathrm{H}, J=8.2$ $\mathrm{Hz}), 6.72(\mathrm{~d}, 1 \mathrm{H}, J=2.1 \mathrm{~Hz}), 6.68(\mathrm{dd}, 1 \mathrm{H}, J=8.2,2.1 \mathrm{~Hz})$, $5.94(\mathrm{~s}, 1 \mathrm{H}), 4.33(\mathrm{dd}, 1 \mathrm{H}, J=11.4,4.3 \mathrm{~Hz}), 4.17(\mathrm{dd}, 1 \mathrm{H}, J=$ 11.4, $7.2 \mathrm{~Hz}), 3.83(\mathrm{~s}, 3 \mathrm{H}), 3.73(\mathrm{~s}, 3 \mathrm{H}), 3.08(\mathrm{dd}, 1 \mathrm{H}, J=$ $13.9,4.7 \mathrm{~Hz}), 2.86-2.82(\mathrm{~m}, 1 \mathrm{H}), 2.65(\mathrm{dd}, 1 \mathrm{H}, J=13.9,10.2$ $\mathrm{Hz}) .{ }^{13} \mathrm{C}\left\{{ }^{1} \mathrm{H}\right\}$ NMR $\left(150 \mathrm{MHz}, \mathrm{CD}_{3} \mathrm{OD}\right) \delta 199.5,161.1$, $161.1,155.7,147.9,147.7,132.2,129.6,121.4,117.1,112.9$, $102.9,97.0,70.6,61.5,56.4,47.9,33.2$. HRMS (ESI) $\mathrm{m} / z$ : [M $+\mathrm{H}]^{+}$calcd for $\mathrm{C}_{18} \mathrm{H}_{18} \mathrm{O}_{7}$ 347.1131; found, 347.1125.

3-(4-Hydroxybenzyl)-5,8-dimethoxychroman-7-ol (14). (E)-7-(Benzyloxy)-3-(4-(benzyloxy)benzylidene)-5,8-dimethoxychroman-4-one (29a) (30 mg, $0.06 \mathrm{mmol}$ ), and $10 \%$ $\mathrm{Pd} / \mathrm{C}(1.8 \mathrm{mg}, 0.006 \mathrm{mmol})$ in methanol $(2 \mathrm{~mL})$ were placed under an atmosphere of hydrogen. After stirring for $3 \mathrm{~h}$, the mixture was filtered through a Celite pad. After the filtrate was concentrated under reduced pressure, purification of the residue via flash column chromatography on silica gel (ethyl acetate $/ n$-hexane $=1: 1$ ) afforded 3-(4-hydroxybenzyl)-5,8dimethoxychroman-7-ol (14) (17 mg, 93\%). ${ }^{1} \mathrm{H}$ NMR (600 $\left.\mathrm{MHz}, \mathrm{CD}_{3} \mathrm{OD}\right) \delta 7.01(\mathrm{~d}, 2 \mathrm{H}, J=8.5 \mathrm{~Hz}), 6.72(\mathrm{~d}, 2 \mathrm{H}, J=8.5$ $\mathrm{Hz}), 6.03(\mathrm{~s}, 1 \mathrm{H}), 4.11$ (ddd, $1 \mathrm{H}, J=10.5,3.0,1.5 \mathrm{~Hz}), 3.71-$ $3.69(\mathrm{~m}, 4 \mathrm{H}), 3.68(\mathrm{~s}, 3 \mathrm{H}), 2.61(\mathrm{ddd}, 1 \mathrm{H}, J=16.3,5.4,1.5$ $\mathrm{Hz}), 2.58-2.49(\mathrm{~m}, 2 \mathrm{H}), 2.18(\mathrm{dd}, 1 \mathrm{H}, J=16.3,8.5 \mathrm{~Hz})$, 2.14-2.07 (m, 1H). ${ }^{13} \mathrm{C}\left\{{ }^{1} \mathrm{H}\right\}$ NMR $\left(150 \mathrm{MHz}, \mathrm{CD}_{3} \mathrm{OD}\right) \delta$ $156.8,155.1,149.6,149.4,131.7,131.0,130.8,116.2,103.8$, 92.4, 70.78, 61.2, 55.8, 38.2, 35.2, 26.2. HRMS (ESI) $\mathrm{m} / z$ : [M $+\mathrm{H}]^{+}$calcd for $\mathrm{C}_{18} \mathrm{H}_{20} \mathrm{O}_{5}$ 317.1389; found, 317.1386.

3-(4-Hydroxybenzyl)-7,8-dimethoxychroman-5-ol (34). 7-O-Methoxypunctatin (2) (10 mg, $0.03 \mathrm{mmol})$ and $10 \%$ $\mathrm{Pd} / \mathrm{C}(1.4 \mathrm{mg}, 0.01 \mathrm{mmol})$ in methanol $(2 \mathrm{~mL})$ were placed under an atmosphere of hydrogen. After stirring for $24 \mathrm{~h}$, the mixture was filtered through a Celite pad. After the filtrate was concentrated under reduced pressure, purification of the residue via flash column chromatography on silica gel (ethyl acetate $/ n$-hexane $=1: 1)$ afforded 3-(4-hydroxybenzyl)-7,8dimethoxychroman-5-ol (34) (3.4 mg, 35\%). ${ }^{1} \mathrm{H}$ NMR (600 $\left.\mathrm{MHz}, \mathrm{CD}_{3} \mathrm{OD}\right) \delta 7.03(\mathrm{~d}, 2 \mathrm{H}, J=8.4 \mathrm{~Hz}), 6.73(\mathrm{~d}, 2 \mathrm{H}, J=8.4$ $\mathrm{Hz}), 6.06$ (s, 1H), 4.13 (ddd, $1 \mathrm{H}, J=10.4,2.7,1.2 \mathrm{~Hz}$ ), 3.75 (s, 3H), 3.76-3.71 (m, 1H), $3.67(\mathrm{~s}, 3 \mathrm{H}), 2.67$ (ddd, $1 \mathrm{H}, J=$ 16.3, 5.4, $1.1 \mathrm{~Hz}$ ), $2.56(\mathrm{~m}, 2 \mathrm{H}), 2.24(\mathrm{dd}, 1 \mathrm{H}, J=16.3,8.5$ $\mathrm{Hz}), 2.16-2.09(\mathrm{~m}, 1 \mathrm{H}) .{ }^{13} \mathrm{C}\left\{{ }^{1} \mathrm{H}\right\} \operatorname{NMR}\left(150 \mathrm{MHz}, \mathrm{CD}_{3} \mathrm{OD}\right)$ $\delta 156.6,152.3,152.2,149.6,131.5,131.1,130.8,115.9,103.8$, 92.9, 70.6, 61.1, 56.1, 38.0, 35.0, 26.2.

3-(4-Hydroxybenzyl)-5,7-dimethoxychroman-8-ol (36). First step: (E)-3-(4-(benzyloxy)benzylidene)-5,7,8-trimethoxychroman-4-one (19a) (0.15 g, $0.35 \mathrm{mmol})$ and $10 \%$ $\mathrm{Pd} / \mathrm{C}(14 \mathrm{mg}, 0.13 \mathrm{mmol})$ in methanol $(6 \mathrm{~mL})$ were placed under an atmosphere of hydrogen. After stirring for $24 \mathrm{~h}$, the mixture was filtered through a Celite pad. After the filtrate was concentrated under reduced pressure, purification of the residue via flash column chromatography on silica gel (ethyl acetate $/ n$-hexane $=1: 1)$ afforded 4-((5,7,8-trimethoxychro- man-3-yl)methyl)phenol (35) (0.10 g, 90\%). ${ }^{1} \mathrm{H}$ NMR (600 $\left.\mathrm{MHz}_{\mathrm{CDCl}}\right) \delta 7.05(\mathrm{~d}, 2 \mathrm{H}, J=8.2 \mathrm{~Hz}), 6.77(\mathrm{~d}, 2 \mathrm{H}, J=8.5$ $\mathrm{Hz}$ ), 6.09 (s, $1 \mathrm{H}), 5.07$ (br s, $1 \mathrm{H}), 4.21$ (ddd, $1 \mathrm{H}, J=10.5,3.0$, $1.6 \mathrm{~Hz}), 3.86(\mathrm{~s}, 3 \mathrm{H}), 3.79(\mathrm{~s}, 3 \mathrm{H}), 3.79-3.74(\mathrm{~m}, 4 \mathrm{H}), 2.72$ (ddd, $1 \mathrm{H}, J=16.3,5.3,1.6 \mathrm{~Hz}), 2.59$ (d, $2 \mathrm{H}, J=7.2 \mathrm{~Hz}$ ), 2.26 $(\mathrm{dd} 1 \mathrm{H}, J=16.3,8.6 \mathrm{~Hz}),, 2.23-2.16(\mathrm{~m}, 1 \mathrm{H}) .{ }^{13} \mathrm{C}\left\{{ }^{1} \mathrm{H}\right\} \mathrm{NMR}$ $\left(150 \mathrm{MHz}, \mathrm{CDCl}_{3}\right) \delta 154.2,153.6,151.4,148.9,131.6,130.2$, $115.4,104.4,88.9,70.0,61.2,56.5,55.7,37.5,33.7,25.5$. HRMS (ESI) $m / z:[\mathrm{M}+\mathrm{H}]^{+}$calcd for $\mathrm{C}_{19} \mathrm{H}_{22} \mathrm{O}_{5} 331.1545$; found, 331.1540. Second step: To a solution of $4-((5,7,8-$ trimethoxychroman-3-yl)methyl)phenol (35) (0.10 g, 0.31 $\mathrm{mmol})$ in $\mathrm{CH}_{2} \mathrm{Cl}_{2}(3 \mathrm{~mL})$ was added boron trichloride (1.0 $\mathrm{mL}, 1.0 \mathrm{M}$ solution in $\mathrm{CH}_{2} \mathrm{Cl}_{2}$ ) at $0{ }^{\circ} \mathrm{C}$. After stirring for $3 \mathrm{~h}$ at ambient temperature, the reaction mixture was diluted with $\mathrm{CH}_{2} \mathrm{Cl}_{2}$, washed with water, dried over anhydrous $\mathrm{Na}_{2} \mathrm{SO}_{4}$, and concentrated under reduced pressure. The residue was purified by flash column chromatography on silica gel (ethyl acetate $/ n$-hexane $=1: 1$ ) to afford 3-(4-hydroxybenzyl)-5,7dimethoxychroman-8-ol (36) (67 mg, 68\%). ${ }^{1} \mathrm{H}$ NMR $(600$ $\left.\mathrm{MHz}, \mathrm{CD}_{3} \mathrm{OD}\right) \delta 7.02(\mathrm{~d}, 2 \mathrm{H}, J=8.4 \mathrm{~Hz}), 6.72(\mathrm{~d}, 2 \mathrm{H}, J=8.4$ $\mathrm{Hz}), 6.20(\mathrm{~s}, 1 \mathrm{H}), 4.15$ (ddd, $1 \mathrm{H}, J=10.5,2.9,1.5 \mathrm{~Hz}), 3.82$ (s, 3H), $3.73(\mathrm{~s}, 3 \mathrm{H}), 3.75-3.70(\mathrm{~m}, 1 \mathrm{H}), 2.66(\mathrm{ddd}, 1 \mathrm{H}, J=$ $16.5,5.4,1.3 \mathrm{~Hz}), 2.58-2.52(\mathrm{~m}, 2 \mathrm{H}), 2.22(\mathrm{dd}, 1 \mathrm{H}, J=16.5$, $8.7 \mathrm{~Hz}), 2.12(\mathrm{~m}, 1 \mathrm{H}) .{ }^{13} \mathrm{C}\left\{{ }^{1} \mathrm{H}\right\} \mathrm{NMR}\left(150 \mathrm{MHz}, \mathrm{CD}_{3} \mathrm{OD}\right) \delta$ 156.8 , 151.7, 147.2, 144.9, 131.7, 131.0, 129.6, 116.2, 105.4, 90.6, 70.9, 57.0, 56.2, 38.3, 35.3, 26.3 .

\section{ASSOCIATED CONTENT}

\section{Supporting Information}

The Supporting Information is available free of charge at https://pubs.acs.org/doi/10.1021/acsomega.0c00932.

${ }^{1} \mathrm{H}$ and ${ }^{13} \mathrm{C}\left\{{ }^{1} \mathrm{H}\right\}$ NMR spectral data and HPLC analysis for synthesized chiral compounds; 2D NMR (HMBC) of 14, 34, and 36 (PDF)

\section{AUTHOR INFORMATION}

\section{Corresponding Author}

Seung-Yong Seo - College of Pharmacy, Gachon University, Incheon 21936, Republic of Korea; (1) orcid.org/0000-00022672-4707; Phone: (+82) 32-820-4949; Email: syseo@ gachon.ac.kr; Fax: (+82) 32-820-4829

\section{Authors}

Sangil Kwon - College of Pharmacy, Gachon University, Incheon 21936, Republic of Korea

Sanha Lee - College of Pharmacy, Gachon University, Incheon 21936, Republic of Korea

Myunghoe Heo - College of Pharmacy, Gachon University, Incheon 21936, Republic of Korea

Bit Lee - College of Pharmacy, Gachon University, Incheon 21936, Republic of Korea

Xiang Fei - College of Pharmacy, Gachon University, Incheon 21936, Republic of Korea; 이이. org/0000-0001-96371989

Timothy W. Corson - Eugene and Marilyn Glick Eye Institute, Department of Ophthalmology, Department of Biochemistry and Molecular Biology, Department of Pharmacology and Toxicology, and Melvin and Bren Simon Cancer Center, Indiana University School of Medicine, Indianapolis, Indiana 46202, United States; 이이이.org/0000-0002-1402-7875

Complete contact information is available at:

https://pubs.acs.org/10.1021/acsomega.0c00932 


\section{Notes}

The authors declare no competing financial interest.

\section{ACKNOWLEDGMENTS}

This work was supported by grants from the National Eye Institute (Grant number R01EY025641) and the BrightFocus Foundation (Grant number M2015301) to TWC and grants from the National Research Foundation funded by the Korean government, MSIP (NRF-2014M3C1A3054139 and NRF2017M3A9C8027781), and the Korea Health Technology R\&D Project through the Korea Health Industry Development Institute (KHIDI), funded by the Ministry of Health \& Welfare (Grant Number HI14C1135) to S.-Y.S.

\section{REFERENCES}

(1) Lin, L. G.; Liu, Q. Y.; Ye, Y. Naturally occurring homoisoflavonoids and their pharmacological activities. Planta Med. 2014, 80, 1053-1066.

(2) Mulholland, D. A.; Schwikkard, S. L.; Crouch, N. R. The chemistry and biological activity of the Hyacinthaceae. Nat. Prod. Rep. 2013, 30, 1165-1210.

(3) Abegaz, B. M.; Kinfe, H. H. Naturally Occurring Homoisoflavonoids: Phytochemistry, Biological Activities, and Synthesis (Part II). Nat. Prod. Commun. 2019, 14, 475-498.

(4) du Toit, K.; Drewes, S. E.; Bodenstein, J. The chemical structures, plant origins, ethnobotany and biological activities of homoisoflavanones. Nat. Prod. Res. 2010, 24, 457-490.

(5) Lee, B.; Basavarajappa, H. D.; Sulaiman, R. S.; Fei, X.; Seo, S. Y.; Corson, T. W. The first synthesis of the antiangiogenic homoisoflavanone, cremastranone. Org. Biomol. Chem. 2014, 12, 7673-7677. (6) Lee, H. J.; Yuan, Y.; Rhee, I.; Corson, T. W.; Seo, S.-Y. Synthesis of natural homoisoflavonoids having either 5,7-dihydroxy-6-methoxy or 7-hydroxy-5,6-dimethoxy groups. Molecules 2016, 21, 1058-1067.

(7) Basavarajappa, H. D.; Lee, B.; Fei, X.; Lim, D.; Callaghan, B.; Mund, J. A.; Case, J.; Rajashekhar, G.; Seo, S. Y.; Corson, T. W. Synthesis and mechanistic studies of a novel homoisoflavanone inhibitor of endothelial cell growth. PLoS One 2014, 9, No. e95694.

(8) Basavarajappa, H. D.; Lee, B.; Lee, H. J.; Sulaiman, R. S.; An, H. C.; Magaña, C.; Shadmand, M.; Vayl, A.; Rajashekhar, G.; Kim, E.-Y.; Suh, Y.-G.; Lee, K.; Seo, S.-Y.; Corson, T. W. Synthesis and biological evaluation of novel homoisoflavonoids for retinal neovascularization. J. Med. Chem. 2015, 58, 5015-5027.

(9) Sulaiman, R. S.; Merrigan, S.; Quigley, J.; Qi, X.; Lee, B.; Boulton, M. E.; Kennedy, B.; Seo, S.-Y.; Corson, T. W. A novel small molecule ameliorates ocular neovascularisation and synergises with anti-VEGF therapy. Sci. Rep. 2016, 6, No. 25509.

(10) Lee, B.; Sun, W.; Lee, H.; Basavarajappa, H.; Sulaiman, R. S.; Sishtla, K.; Fei, X.; Corson, T. W.; Seo, S.-Y. Design, synthesis and biological evaluation of photoaffinity probes of antiangiogenic homoisoflavonoids. Bioorg. Med. Chem. Lett. 2016, 26, 4277-4281.

(11) Basavarajappa, H. D.; Sulaiman, R. S.; Qi, X.; Shetty, T.; Sheik Pran Babu, S.; Sishtla, K. L.; Lee, B.; Quigley, J.; Alkhairy, S.; Briggs, C. M.; Gupta, K.; Tang, B.; Shadmand, M.; Grant, M. B.; Boulton, M. E.; Seo, S.-Y.; Corson, T. W. Ferrochelatase is a therapeutic target for ocular neovascularization. EMBO Mol. Med. 2017, 9, 786-801.

(12) Sulaiman, R. S.; Park, B.; Sheik Pran Babu, S. P.; Si, Y.; Kharwadkar, R.; Mitter, S. K.; Lee, B.; Sun, W.; Qi, X.; Boulton, M. E.; Meroueh, S. O.; Fei, X.; Seo, S.-Y.; Corson, T. W. Chemical proteomics reveals soluble epoxide hydrolase as a therapeutic target for ocular neovascularization. ACS Chem. Biol. 2018, 13, 45-52.

(13) Heo, M.; Lee, B.; Sishtla, K.; Fei, X.; Lee, S.; Park, S.; Yuan, Y.; Lee, S.; Kwon, S.; Lee, J.; Kim, S.; Corson, T. W.; Seo, S.-Y. Enantioselective Synthesis of Homoisoflavanones by Asymmetric Transfer Hydrogenation and Their Biological Evaluation for Antiangiogenic Activity. J. Org. Chem. 2019, 84, 9995-10011.

(14) Alali, F.; El-Elimat, T.; Albataineh, H.; Al-Balas, Q.; AlGharaibeh, M.; Falkinham, J. O., III; Chen, W. L.; Swanson, S. M.;
Oberlies, N. H. Cytotoxic Homoisoflavones from the Bulbs of Bellevalia eigii. J. Nat. Prod. 2015, 78, 1708-1715.

(15) Du Toit, K.; Elorashi, E. E.; Malan, S. F.; Drewes, S. E.; van Staden, J.; Crouch, N. R.; Mulholland, D. A. Anti-inflammatory activity and QSAR studies of compounds isolated from Hyacinthaceae species and Tachiadenus longiflorus Griseb. (Gentianaceae). Bioorg. Med. Chem. 2005, 13, 2561-2568.

(16) Ngamga, D.; Bipa, J.; Lebatha, P.; Hiza, C.; Mutanyatta, J.; Bezabih, M.-T.; Tane, P.; Abegaz, B. M. Isoquinoline Alkaloids and Homoisoflavonoids from Drimiopsis barteri Bak and D. burkei Bak. Nat. Prod. Commun. 2008, 3, 769-777.

(17) Sidwell, W. T. L.; Tamm, C. Homo-isoflavones. II. Isolation and structure of 4'-O-methyl-punctatin, autumnalin and 3,9-dihydroautumnalin. Tetrahedron Lett. 1970, 11, 475-478.

(18) Finckh, R. E.; Tamm, C. The homo-isoflavones III. isolation and structure of punctatin, 3,9-dihydro-punctatin, 4'-O-methyl-3,9dihydro-punctatin, $4^{\prime}$-demethyl-eucomin and $4^{\prime}$-demethyl-5-O-methyl-3,9-dihydro-eucomin. Experientia 1970, 26, 472-473.

(19) Koorbanally, C.; Crouch, N. R.; Langlois, A.; Du Toit, K.; Mulholland, D. A.; Drewes, S. E. Homoisoflavanones and spirocyclic nortriterpenoids from three Eucomis species: E. comosa, E. schijffii and E. pallidiflora subsp. pole-evansii (Hyacinthaceae). S. Afr. J. Bot. 2006, $72,428-433$.

(20) Corsaro, M. M.; Lanzetta, R.; Mancino, A.; Parrilli, M. Homoisoflavanones from Chionodoxa luciliae. Phytochemistry 1992, 31, 1395-1397.

(21) Adinolfi, M.; Barone, G.; Belardini, M.; Lanzetta, R.; Laonigro, G.; Parrilli, M. Homoisoflavanones from Muscari comosum bulbs. Phytochemistry 1985, 24, 2423-2426.

(22) Mutanyatta, J.; Matapa, B. G.; Shushu, D. D.; Abegaz, B. M. Homoisoflavonoids and xanthones from the tubers of wild and in vitro regenerated Ledebouria graminifolia and cytotoxic activities of some of the homoisoflavonoids. Phytochemistry 2003, 62, 797-804.

(23) Xu, X.; Cheng, K.; Cheng, W.; Zhou, T.; Jiang, M.; Xu, J. Isolation and characterization of homoisoflavonoids from Dracaena cochinchinensis and their osteogenic activities in mouse mesenchymal stem cells. J. Pharm. Biomed. Anal. 2016, 129, 466-472.

(24) Farkas, L.; Gottsegen, A.; Nogradi, M.; Strelisky, J. Synthesis of homoisoflavanones. II. Constituents of Eucomis autumnalis and E. punctata. Tetrahedron 1971, 27, 5049-5054.

(25) (a) Qin, T.; Metz, P. Enantioselective synthesis of isoflavanones by catalytic dynamic kinetic resolution. Org. Lett. 2017, 19, 29812984. (b) Ashley, E. R.; Sherer, E. C.; Pio, B.; Orr, R. K.; Ruck, R. T. Ruthenium-catalyzed dynamic kinetic resolution asymmetric transfer hydrogenation of $\beta$-chromanones by an elimination-induced racemization mechanism. ACS Catal. 2017, 7, 1446-1451.

(26) (a) Fujii, A.; Hashiguchi, S.; Uematsu, N.; Ikariya, T.; Noyori, R. Ruthenium(II)-Catalyzed Asymmetric Transfer Hydrogenation of Ketones Using a Formic Acid-Triethylamine Mixture. J. Am. Chem. Soc. 1996, 118, 2521-2522. (b) Ohkuma, T.; Ishii, D.; Takeno, H.; Noyori, R. Asymmetric hydrogenation of amino ketones using chiral $\mathrm{RuCl}_{2}$ (diphophine)(1,2-diamine) complexes. J. Am. Chem. Soc. 2000, $122,6510-6511$

(27) Ley, S. V.; Norman, J.; Griffith, W. P.; Marsden, S. P. Tetrapropylammonium Perruthenate, $\mathrm{Pr}_{4} \mathrm{~N}^{+} \mathrm{RuO}_{4}{ }^{-}$, TPAP: A Catalytic Oxidant for Organic Synthesis. Synthesis 1994, 639-666.

(28) Adinolfi, M.; Lanzetta, R.; Laonigro, G.; Parrilli, M.; Breitmaier, E. ${ }^{1} \mathrm{H}$ and ${ }^{13} \mathrm{C}$ Chemical Shift Assignments of Homoisoflavanones. Magn. Reson. Chem. 1986, 24, 663-666.

(29) Carvalho, C. F.; Russo, A. V.; Sargent, M. V. Boron Trichloride as a Selective Demethylating Agent for Hindered Ethers: a Synthesis of the Phytoalexins and P-Pyrufuran, a Synthesis of Tri-Omethylleprolomin and its Demethylation. Aust. J. Chem. 1985, 38, $777-792$

(30) Heller, W.; Tamm, C. 5,7-Dihydroxy-8-methoxy-chroman-4-on aus dem Zwiebelwachs von Eucomis comosa. Helv. Chim. Acta 1978, $61,1257-1261$. 